\title{
Product Fragmentation and Trade Patterns in East Asia
}

\author{
Prema-chandra Athukorala
}

October 2003

Working Paper No. 2003/21 


\title{
Product Fragmentation and Trade Patterns in East Asia
}

\author{
Prema-chandra Athukorala \\ Division of Economics \\ Research School of Pacific and Asian Studies \\ Australian National University \\ Email: prema-chandra.Athukorala@anu.edu.au
}

Abstract: International product fragmentation - the cross-border dispersion of component production/assembly within vertically integrated production processes - is an important feature of the deepening structural interdependence of the world economy. This paper examines the implications of this phenomenon for global and regional trade patterns, with special emphasis on countries in East Asia, using a new data set culled from the UN trade database. It is found that, while 'fragmentation trade' has generally grown faster than total world trade in manufacturing, the degree of dependence of East Asia on this new form of international specialisation is proportionately larger compared to North America and Europe. The upshot is that international product fragmentation has made the East Asian growth dynamism increasingly reliant on extra-regional trade, strengthening the case for a global, rather than a regional, approach to trade and investment policymaking.

JEL classification: $\quad$ F15, F23, O53

Key words: $\quad$ product fragmentation, globalisation, multinational enterprises 


\section{Product Fragmentation and Trade Patterns in East Asia ${ }^{*}$}

\section{Introduction}

Globalisation - the deepening structural interdependence of the world economy-is one of the most discussed subjects in the development policy debate in the last few decades. An important feature of the on-going process of globalisation is product fragmentation the cross-border dispersion of component production/assembly within vertically integrated production processes ${ }^{1}$ For example, Japanese car producers export engine parts to their affiliates in Thailand where they are assembled into engines using some other components procured from other countries in the region and export back to Japan and other third-country markets. Computer producers in the US increasingly rely on worldwide procurement networks spanning the globe for the assembly/production of most of the components embodied in the final product, and more recently some producers have begun to shift even final stage assembly to some countries in Asia. German camera producers are now engaged only in design and marketing tasks, after shifting most segments of the production process to Japan and other Asian countries.

There is a sizeable theoretical literature examining causes and modalities of international product fragmentation, and its implications for trade flow analysis and trade policy (eg. Baldwin 2001; Cantwell 1994; Deardorff 2001; Jones 2000, Jones and Kierzkowski 2001a, 2001b, \& 2001c, Venables 1999). This literature postulates that this form of international exchange is much more sensitive to inter-country differences in cost

\footnotetext{
* This paper is based on research in progress for the Regional Economic Monitoring Unit, Asian Development Bank. Comments on an earlier version of the paper by Christopher Findlay, Ross Garnaut, Hal Hill, Jamie MacKie and David Vines are gratefully acknowledged. Thanks are also due to Azis Marpudin for a meticulous job done in extracting data from the UN data tapes, and to Nobuaki Yamashita and Junthathip Jongwanich for superb research assistance.

${ }^{1}$ This phenomenon has gone under alternative names, such as 'Vertical specialisation' (Hummels, Rapoport and Yi 2001, Irwin 2002), 'slicing the value chain' (Krugman 1995), 'international production sharing' (Ng and Yeats 2001, Yeats 2001), and 'outsourcing' (Rangan and Lawrence 1999, Hanson, Raymond and Slaughter 2002). Following Jones (2000), Jones and Kierzkowski (2001a, 2001b, \& 2001c), Venables 1999, Baldwin 2001 and others we stick to the term, 'product fragmentation' in this paper.
} 
of production (rather than relative cost differentials among production activities within a given country) compared to trade in finished goods, and that increased reliance on firms on outsourcing of components has implications for the distribution of gains from trade between labour and capital. As for the study of international economic relations, it is predicted that the growing importance of fragmentation trade trade tends to erode the relevance of relative prices (normally of 'final goods', in most empirical applications) and world income as explanatory variables in trade flow analysis and global trade modelling.

Applied trade economists have, however, been rather slow in responding to this new form of international specialisation. Trade flow analysis is still based on the traditional notion of horizontal specialisation scenario in which countries trade goods that are produced from start to finish in just one country. Our understanding of the phenomenon of product fragmentation and its implications for international production and trade patterns is largely confined to case-study evidence coming from the literature on multinational enterprises (eg. Borrus 1997 and 1999; Crosby and Nakamori 1991; Dobson and Chia 1997; Naughton 1999, McKendrick, Doner and Haggards 2000). ${ }^{2}$ This gap in the empirical trade literature is due primarily to the nature of readily accessible data on trade flows. The recent revisions to the UN trade data system have provided for countries to report data at a level suitable for separating 'fragmentation trade' from the traditional form of commodity trade ('final trade'). However, this distinction has not yet been built into the readily available (published) data tabulations arising from the UN reporting system.

This paper aims to examine the extent, trends and patterns of fragmentation trade trade, and the implications of this new form of international specialisation for analysing

\footnotetext{
${ }^{2}$ Notable exceptions are Hummels, Rapoport and YI (2001), Yeats (2001) and Ng and Yeats (2001). The first of these three studies looks at importance of product fragmentation for economic performance in selected OECD countries by examining the dependence of domestic production in imported intermediate. inputs. The latter two studies examines the relative importance of trade in parts and components in total machinery trade in OECD countries and East Asia respectively, using OECD country import trade data (based on Revision 2 of the Standard International Trade Classification).
} 
regional trade patterns, with special emphasis on the trade performance of countries in East Asia. The study is based on a systematic separation of trade in parts and components from total trade flows using UN trade data tapes. The East Asian experience is examined in the wider global setting, paying due attention to the involvement of other major trading nations/regions in assembly activities in the region.

There is a vast literature based on the standard trade data which points unequivocally to a persistent increase in intra-regional trade in East Asia (including as well as excluding Japan) form about the early 1980s (eg. Kwan 2001, Drysdale and Garnaut 1997, and. Frankel and Wei 1997, Petri 1993). This evidence figures prominently in the current regional debate on forming regional trading arrangements covering some or all countries in East Asia. In particular, the proponents of the proposal by the Malaysian Prime Minister Mahathir to form an East Asian Economic Caucus (EAEC) often refer to deepening economic interdependence reflected in intra-regional trade as evidence of its likely success. Increasing trade integration is also cited as an indicator of the potential benefits of monetary integration in the region (Kwan 2001). The hypothesis underpinning our analysis is that, in a context where component trade is growing rapidly, the conventional trade flow analysis, which is based on the (implicit) assumption that countries always trade in 'goods', can lead to misleading inferences as to the nature and extent of trade integration among countries.

The paper is structured as follows: Section 2 provides an overview of the phenomenon of international product fragmentation and related policy issues to set the stage for the ensuing analysis. Section 3 discusses the procedure followed in extracting data from the UN trade data tapes and data quality. Section 4 examines the nature and extent of global trade in components and East Asia's role in this form of trade specialisation. Section 5 deals with the implications of the rapid expansion of product fragmentation for analysing intra- and extra-regional patterns of economic integration of East Asia. The final section presents the key policy inferences. 


\section{International Product Fragmentation: An Introduction}

This section is in two parts. It first provides an overview of the process of international product fragmentation and its determinants. This is followed by a succinct discussion of the policy implications this new form of international specialisation for trade and investment policy in developing countries.

\section{The Process}

The location in developing countries of relatively labour intensive component production and assembly within vertically integrated international industries has been an important feature of the international division of labour since about the late 1960s. The process started in electronics and garment industries and subsequently spread to many other industries (Sharpton 1975, Helleiner 1973, Feenstra 1998). The transfer abroad of component assembly operations now occurs in many industries where the technology of production permits the separation of labour intensive components from other stages of production. Assembly operations in the electronic industry (in particular assembly of semi-conductor devices, hard disk drives etc) are by far the most important. The other industries with significant assembly operations located in developing countries are electrical appliances, automobile parts, electrical machinery and optical products, musical equipment, watches, and cameras. In general, industries that have the potential to break up the production process to minimise the transport cost involved are more likely to move to peripheral countries than other heavy industries.

International product fragmentation allows companies to unbundle stages of production so that each stage can be relocated in countries in which the intensively used inputs are cheap. The nature of factor intensity of the given segments and the relative prices of factors in comparison with their productivity jointly determine which country produces what components. Even then international product fragmentation will not occur if the extra costs associated with fragmentation outweigh the gain from the lower costs of the activity abroad. These extra costs may include transportation, communication, and 
other costs involved in coordinate the activity with what is still being done in the home country.

In this context, the expansion of international product fragmentation as a new facet of international production has been largely underpinned by three mutually reinforcing development over the past few decades. First, rapid advancements in production technology has enabled the industry to slice up the value chain into finer, 'portable', components. Second, technological innovations in communication and transportation have shrunk the distance that once separated the world's nations, and improved speed, efficiency and economy of coordinating geographically dispersed production process. This has facilitated establishment of 'services links' to combine various fragments of the production process in a timely and cost efficient manner (Jones and Kierzkowski 2001a). Third, liberalisation policy reforms in both home and host countries have considerably removed barriers to trade and investment.

There is an important two-way link between improvement in production/communication technology and the expansion of fragmentation-based specialisation within global industries. The latter results in lowering cost of production and rapid market penetration of the final products through enhanced price competitiveness. Scale economies resulting in market expansion in turn encourage new technological efforts, enabling further product fragmentation. This two-way link has set the stage for 'fragmentation trade' to increase more rapidly compared to conventional commodity-based trade (Jones 2000).

At the early stage, an important policy affecting the relocation of assembly activities was a provision in the tariff structures of the US and other major industrialised countries (ICs) that allows companies to export material for processing overseas and to reimport the finished products, paying tariff only on the value added abroad (not the exported intermediates) (Sharpton 1975, Helleiner 1973). The importance of these tariff concessions as a factor in promoting global sourcing has, however, significantly been 
diminished over the years by the process of investment and trade liberalisation in ICs and regional economic integration agreements.

Production outsourcing practices were initiated by multinational enterprises (MNEs ) based in the USA. The involvement of Japanese and Western European MNEs in outsourcing began to gain importance from the late 1970s. More recently, MNEs from more advanced developing countries, notably those from the East Asian newly industrialised countries (NIEs) have also joined this process of internationalisation of production. In response to rapid domestic wage increases, the growing reluctance of domestic labour to engage in low paid blue-colour employment, and stringent restrictions on the importation of labour, firms in the electronic industry and other durable consumer goods industries in NIEs in East Asia have begun to produce components and sub-assembly activities in neighbouring countries where labour costs are still low. Conventionally, international product fragmentation took the form of a MNE building a subsidiary abroad to perform some of the functions that it once did at home. But as production operations in host countries became firmly rooted, MNEs have begun to subcontract some activities to pure local firms to which they provide detailed specifications and even fragments of their own technology. In recent years, outsourcing practices have begun to spread beyond the domain of MNEs. Many companies, which are not parts of MNE networks, now procure components globally through arm's-length trade. Technological innovations in communication (in particular the Internet) have reduced cost of outsourcing, particularly through reduced research costs. The process has also been facilitated by the 'standardization of some components, as discussed before (standardisation of components increases outsourcing flexibility.. However, the bulk of fragmentation trade still takes place under the rubric of MNEs (Rangan and Lawrence 1999; Hanson, Mataloni and Slaughter 2001, Urata 2001).

At the formative stage of international product fragmentation, outsourcing took predominantly the form of locating small fragments of the production process in a low cost country and reimporting the assembled components to be incorporated in the final product. Over time the fragmentation process has expanded to involve many countries in 
the assembly process at different stages, resulting in multiple border crossing of product fragments before getting incorporated in the final product. Recent years have witnessed two other important developments in the process, setting the stage for rapid expansion in the share of fragmenta ion-based trade in world trade.

First, some fragments of the production process in certain industries have become 'standard fragments' which can be effectively used in a number of products. Examples include, long-lasting cellular batteries originally developed by computer produces and now widely used in cellular phones and electronic organisers; transmitters which are used not only in radios (as originally designed) but also in PCs and missiles; and electronic chips which have spread beyond the computer industry into consumer electronics, motor vehicle production and many other product sectors (Jones 2000; Jones and Kierzkowski 2001a; Brown, Deardroff and Stern 2003).

Second, there has been a noteworthy expansion of the coverage of global assembly operations from component production and assembly to assembly of final products (eg computers, cameras, TV sets and motor cars). In final assembly, labour costs, while significant, are of secondary importance compared to the availability of world-class operator, technical and managerial skills; a good domestic basis of supplies and services; relatively free access to world-priced inputs including capital; and excellent infrastructure. In other words, the locational decisions of MNEs in this sphere depend on the availability of a wider array of complementary inputs that enable their facilities to be efficient by world standards. Also, given the heavy initial fixed costs, MNEs are hesitant to establish overseas plants in final assembly without considerable first-hand commercial experience in the host country. For these reasons overseas production units of MNEs involved in such final stage assembly are located in other industrialised countries or in more advanced newly industrialising countries (NIEs). ${ }^{3}$

\footnotetext{
${ }^{3}$ However, in recent years China has emerged as an important location for final assembly in many product lines largely because of the vast domestic market for these products which naturally reduces the risk of covering the initial establishment costs (Borrus 1997)
} 


\section{Policy Issues}

Fragmentation of production and the emergence of new forms of international trade have begun to open up new opportunities for developing countries and transition economies to participate in a finer international division of labour. For instance, a country need not set up a motor vehicle plant to benefit from the growth of the automobile industry: it is enough to be competitive in the production of a single part. At the early stage of international product fragmentation, some observers were sceptical about prospects for developing countries to rely on this form of international specialisation for export expansion. They predicted that the process would be reversed because of rapid automation of production processes in developed countries (eg Frobel et al. 1980, Cantwell 1994). However, in many high-tech industries (notably electronics and electrical products) rapid innovation and continuous technical change, which bring about a constant cycle of change and obsolescence, are formidable constraints to rapid automation as an alternative to offshore assembly. Therefore, the indications are that this form of internationalisation of production will continue to expand, providing countries the opportunity to find new niches for labour intensive, export-oriented production (depending of corse on their ability to provide an enabling domestic economic environment). Thus, international product fragmentation presents a challenge to those who believe in the so-called 'fallacy of composition' argument against export-led industrialisation in developing countries. '

Fragmentation-based specialisation has the potential to magnify the gains from lowering policy barriers at border (such as tariff and quantitative restriction), which restrict trade. In this sequential mode of production, activities at separate stages have thin margins, and therefore are highly sensitive to even small tariffs. When a good crosses multiple borders, even low tariff rates are magnified as they are repeatedly applied to the goods-in-process. Thus in the presence of growing opportunities for fragmentation trade trade, further trade liberalisation could yield substantial gains (provided of course the other preconditions such political stability, sound macroeconomic management, and availability of labour of required quality at competitive wage levels and labour market flexibility are met). To be effective, opening up of the border needs to be appropriately 
combined with policy initiatives to dismantle/harmonise barriers behind borders, such as regulations, national standards, competition policies, and government procurements.

Fragmentation trade also has implications for the debate on the gains from regional trading agreements and other regional economic groupings. Trade distorting measures involved in regional trade agreements (RTAs) are detrimental to gains from fragmentation trade (Baldwin 2001). In particular, such trade can bee burdened by transaction costs associated with rules in RTAs. Moreover, maintaining barriers to trade against non-members (while allowing free trade among members) can thwart 'natural' expansion of fragmentation trade across countries.

As competitive firms in market economies shift from just-in-case production and inventory systems to just-in-time organization, the supply of parts, components, and subsystems are required to respond rapidly and with flexibility to changing market conditions. There is a need for rapid and elastic production response for success. Thus international harmonisation of regulations that affect labour and environment standards are important for enabling a country to participate effectively in fragmentation-based global specialisation.

International product fragmentation has made the linkage between national policies relating to foreign direct investment (FDI), and trade and industrialisation stronger than ever. As already noted, MNEs are the key players in worldwide assembly operations. Moreover, opportunities for the development of arms-length production relationships in a given country depend crucially on the initial entry of MNEs. Therefore, to realise trade gains from fragmentation-based specialisation, countries have to place greater emphasis on eliminating restrictions on foreign direct investment (FDI). In the absence of a conducive policy setting which enables MNEs to invest freely in foreign countries, vertical specialisation based trade may not occur. 


\section{Data}

This study makes use of data extracted from the UN trade data tapes based on the Revision 3 of the Standard International Trade Classification (SITC, Rev 3). In its original form (SITC, Rev 1), the UN trade data reporting system did not provide for separating fragmentation trade trade (parts and components) from final manufactured goods. The SITC Revision 2 introduced in the late 1970s (and implemented by most countries only in the early 1980s) adopted a more detailed commodity classification, which provided for separation of parts and components within the machinery and transport sector (SITC 7). About 60 individual three, four- and five-digit groups within SITC 7 consisted solely of components of manufactured equipment to be assembled. There were however considerable overlap between some advanced-stage assembly activities and related final goods within the sector in the Revision 2, which made it difficult to separate fragmentation trade trade from total trade (Ng and Yeats 2001) ${ }^{4}$.

SITC Revision 3, which was introduced in the mid-1980s, marked a significant improvement over Revision 2. In addition to redressing overlaps within SITC 7, this new version of SITC provided for separation of parts and components trade in the 'miscellaneous goods' sector (SITC 8). These two sectors together accounted for around $70 \%$ of total world trade during the period under study. ${ }^{5}$ The list of parts and components identified at the 5-digit level for these two sectors, which provides the basis of our empirical analysis, is reported in Appendix A-5. The list contains a total of 225 fivedigit products -168 products belonging to SITC 7 and 57 belonging to SITC 8 .

\footnotetext{
${ }^{4}$ For instance 'television tubes' were not separable from 'TVc' and ' computer processors' were lumped together with 'computers'.

${ }^{5}$ Share of SITC 7 and SITC 8 in total manufacturing trade (SITC 5 though 8)

Exports

$\begin{array}{llll}1992 & 1996 & 2000 & 1992\end{array}$

SITC 7

SITC 8

SITC $7+8$

67.6

53.2

16.7

69.9

Imports

$1992 \quad 1996 \quad 2000$

$53.8 \quad 52.1 \quad 55.1$

$\begin{array}{lll}16.9 & 15.5 & 15\end{array}$

$\begin{array}{lll}70.7 & 67.5 & 70.1\end{array}$
}


It is important to note that, despite its significant improvement over the previous version, SITC Revision 3 does not provide for the construction of data series covering the entire range of activities involving product fragmentation. As already noted, there is evidence that assembly activities are spending beyond SITC 7 and 8 to other areas, in particular pharmaceutical and chemical products (falling under SITC 6). Moreover, assembly activates in software trade, which have recorded impressive expansion in recent years, are lumped together with 'special transactions' under SITC 9. So the tabulations presented here of the magnitude of trade in parts and components are downward biased. However, the magnitude of the bias is unlikely to be substantial because the large share accounted for by SITC 7 and 8 in total manufacturing trade (see Note 4) and also because outsourcing activities are presumably not yet widespread within the other SITC sectors (Yeats 2001, Feenstra 1998).

Although the SITC Revision 3 was introduced in the mid-1980s, there has been a substantial time lag in the implementation of the system by reporting countries. The stating point of our data tabulation is 1992, when countries accounting for over 90 per cent of total manufacturing trade had adopted the new system.

The UN data system does not cover Taiwan (because it is not a UN member). Vietnam has not yet started reporting data under the SITC classification. Singapore has not been reporting data on its bilateral trade with Indonesia from 1964 onwards because of political reasons. In these cases partner- import and export data are based on partner country export and import records respectively.

The prime focus of the empirical analysis of this paper is on East Asia defined to include both Japan and developing countries in the region. The latter include the newly industrialised economies (NIEs) in North Asia (South Korea, Taiwan and Hong Kong), China and members of the Association of Southeast Asian Nations (ASEAN). Aming the ASEAN countries, only the six largest economies - Indonesia, Malaysia, the Philippines, Thailand, Singapore and Vietnam - are covered in the statistical analysis; Brunei, Cambodia, Laos and Myanmar are ignored because of lack of data. The East Asian 
experience is examined in the wider global context, focussing specifically on the comparative experience of that region, and the North American Free Trade Area and the European Union (EU). As regards the commodity coverage, the focus here is on manufacturing trade. Following the standard practice in trade flow analysis, they are defined here to include all goods belonging to SITC sections 5 to 8, net of SITC 68 and 67 (non-ferrous metal).

\section{Trends and Patterns of Product Fragmentation}

World trade in parts and components (henceforth referred to as 'components' for brevity) increased from $\$ 337$ million in 1992 to $\$ 1086$ billion in 2000, recording an annual average growth rate of 18 per cent (Table 1). The share of these products in total world manufacturing trade increased from 21 per cent to 25 per cent between these two years.

The share of East Asia (including Japan) in total world exports of components increased persistently from 34.5 per cent in 1992 to 39.5 per cent in 2000. This is despite a persistent decline in the share accounted for Japan, the dominant economy in the region, from 18 per cent to 13.6 per cent. The share of developing East Asia (East Asia excluding Japan) increased from 16.6 to 26.5 per cent between these two years. Within the group, all reported countries except Singapore have recorded increases in world market share. Rapid increase in assembly exports from China (a five-fold increase in export value, from $\$ 4$ billion to over $\$ 19$ billion, resulting in an increase in market share from 1.1 per cent to 3.0 per cent) is particularly noteworthy. Contrary to the popular perception of 'crowding out the 'rest' by China', this increase has been within an overall increase in exports from other newcomers in the region. Component trade of Vietnam is also growing, but it still accounts for only a minor fraction of regional trade.

On the import side, Japan's share in the world total is much smaller than that on the export side. It has remained largely unchanged around 5 per cent. This reflects the unique role played by Japan in world assembly trade in undertaking assembly activities in overseas locations (mostly in East Asia). When Japan in excluded, market share changes 
of individual East Asian countries on the import side closely mirror those on the export side.

Some observers predicted that the formation of AFTA and the integration of some of the new countries emerged countries from the former Soviet Union with the rest of the Europe could adversely affect the developing East Asia's relative position in world assembly activities (USITC 1999, Kierzkowski 2001). Proximity to industrial countries where final assembly activities are located and relatively low wages by regional standard (though not compared to some the East Asian countries) were considered as added advantages of these countries compared to East Asian countries. Interestingly, this prediction is not supported by the data. World market shares of Mexico and rest of Europe (EUT less EU) have increased, but at a much slower rate than that of developing East Asia.

One possible explanation is that, despite rapid industrialisation for over a decade, the average manufacturing wage in East Asia still remains low compared to Europe and North America (Table 2). Moreover, significant differences in wages among the countries in the region have provided the basis for rapid expansion of intra-regional product sharing system in the region, giving rise to increased cross-border trade in parts and components. Substantial wage differential among countries in the region naturally makes intra-regional specialisation especially profitable. Also, as first comers in this area of international specialisation, countries in East Asia (particularly Singapore, Korea, Taiwan and Malaysia) offer considerable agglomeration advantages for companies that are already located there. Location decisions of MNEs operating in assembly activities are strongly influenced by the presence of other key market players in the given country (Barry and Bradley 1997, Ruane and Gorg 2001). Also, there is a general tendency for MNE affiliates to become increasingly embedded in host countries the longer they are present there and the more conducive the overall investment climate of the host country becomes over time. They may respond sluggishly to relative cost changes once they have invested substantial resources in domestic production facilities and in establishing information links (Rangan and Lawrence 1999). 
In all countries/regions covered in our data tabulations, assembly trade is heavily concentrated in the machinery and transport equipment sector (SITC 7) (Table 3). This sector accounts for over 90 per cent of the combined assembly trade of SITC 7 and SITC 8 (miscellaneous manufacturing). Within SITC 7, both assembly exports and imports of East Asia are heavily concentrated in electronics and electrical industries. The four SITC3 digit industries of parts and accessories of office machinery (SITC 759), telecommunication equipment (SITC 764), apparatus for electrical circuits (SITC 772) and household electrical equipment (SITC 776) account for over 80 per cent of total trade. There is little variation among the individual East Asian countries in terms of trade composition. These electronics and electrical products are also the major areas of activity in other countries/regions. But trade patterns of these countries/regions are characterised by a greater presence of other items such as engines and motors (SITC 714), industrial machinery (SITC 728), internal combustion machines (SITC 713) for which transportation cost is presumably an important consideration for production location. Overall, these differences are consistent with our earlier inference about East Asia's competitive edge in fragmentation-based production in electrical and electronic industries.

Japan has persistently recorded a large trade surplus in assembly trade, reflecting the heavy involvement of Japanese companies in overseas assembly activities to maintain their competitiveness in final trade in third country markets (Table 1, last three columns). This is in contrast to the US whose assembly trade has remained virtually in balance because of the heavy dependence of US firms on overseas assembly of components in order to maintain competitiveness in domestic market (Burros 1999). Among the individual East Asian countries, trade positions of Malaysia, the Philippines, and Singapore have turned from deficits to surpluses in recent years. As we will see later, this reflects growing cross border trade in assembly products within the region.

Tables 4 and 5 present Comparative statistics on the share of components in total manufacturing exports and imports in 1992, 1996 and 2000 and their contribution to growth of manufacturing trade between these years are reported in Tables 4 and 5. . It is 
evident that the degree of dependence of East Asian countries as a group on assembly trade is much higher compared to all other regions in the world. In 2000, components accounted for 32 per cent of total manufacturing exports from developing East Asia, compared to the world average of 23 per cent, 28.1 for NAFTA and 17.1 per cent for the EU. Of the total increment in manufactured exports from East Asia between 1992 and 2000, over two-fifths came from components exports. The comparable figures for NAFTA and the EU were 30 per cent and 21 per cent respective. For South Asia, Africa, Middle East and Latin America (excluding Mexico) both the export share and relative growth contribution estimates are much smaller (less than 10 percent in all cases) compared to these three regions. The patterns on the import sided by and large mirror the export story.

At the individual country level, Malaysia, Philippine, Singapore and Thailand stand out in developing East Asia for their heavy dependence on product fragmentation for export dynamism. The share of components in total manufacturing exports has increased rapidly in these four countries, reaching over 50 per cent fin Malaysia, Philippine and Singapore and over 35 per cent in Thailand by 2000. Between 1992 and 2000, components exports accounted for 74 per cent of total export increment in the Philippines, 64 per cent in Singapore, 54 per cent in Malaysia and 47 per cent in Thailand. Between these two years, the share of components in total manufacturing exports more than doubled in China (from 6.7 per cent to 14.5\%) and tripled in Vietnam ( 2.5 per cent to 8.7 per cent). However, in both countries the relative contribution of these exports to increment in total manufacturing exports between the two years remained modest (18 per cent in China and 8.7 per cent in Vietnam) because of the low starting bases. Interestingly even in Taiwan and Korea, the relative importance of components in total manufacturing exports (and imports) has increased over the years, contradicting the popular belief that these countries had shifted palpably from assembly activities to final good production over the years. 


\section{Fragmentation and Trade Patterns}

In the previous section we observed growing importance of fragmentation trade trade for trade expansion in East Asia relative to the overall global experience and experiences of countries in other major regions. The purpose of this section is to examine intra-regional trade patters in the region by explicitly taking into account this important development in the economic landscape.

The discussion is based on data on regional bilateral trade flows reported in Tables 5 through 8 . Table 5 depicts regional patterns of trade in total manufacturing and in total manufacturing net of parts and components ('net trade'). Table 6 reports percentage shares of parts and comments in bilateral flows of total manufacturing exports. Growth rates of manufacturing trade during 1992-2000 and the percentage contribution of component trade to growth in trade between the two years are reported in Table 7. Table 8 reports bilateral trade balances (measures as a percentage of exports) of total manufacturing, components and net trade. The same data disaggregated for the individual economies in East Asia are reported in the corresponding four tables (Table A1 through A-4) in the Statistical Appendix. A number of interesting inferences emerge from a careful comparative treatment of data presented in these tables.

In terms of the conventionally used trade data, intra-regional manufacturing trade (export + imports) in East Asia is significant and growing rapidly (Table 5, panel B1). The share of total intra-regional trade in East Asia increased from 47.1 per cent in 1992 to 52.3 per cent in 2000. Intra-regional trade in developing East Asia increased from 36.5 per cent to 39.4 per cent between these two years. For ASEAN the magnitude of these figures is much smaller, but they point to an impressive, persistent increase over the years (from 18.2 per cent to 23.6 per cent). Interestingly, in all three years (1992, 1996 and 2002) for which data are reported in Table 5, the degree of intra-regional trade in East Asia (including Japan) is significant larger compared to the comparable estimates for NAFTA (East Asia: 47.1\%; 51.5\% and 52.3\%; NAFTA: 39.2, 43.1\%, 46.3\%). The estimates for the EU are larger in magnitude $(70.8 \%, 61.0 \%, 58.2 \%)$, but they have been 
falling over the years unlike in East Asia. All in all, in terms of conventionally used trade data the on-going process of regional integration in East Asia looks remarkable. However, these figures need to be interpreted cautiously for two reasons.

First, unlike in EU and NAFTA, the East Asian intra-regional trade ratio camouflages a significant asymmetry in regional trade patterns on import and export sides. In 2000, intra-regional import flows amounted to 64 per cent of total manufacturing imports of East Asia, up from 59 percent in 1995. Interregional share in total regional exports was however significantly lower, amounting to around 45 per cent in both years (Table 5, panel A and B). In other words, the region is much more heavily developed on extra-regional trade for its growth dynamism than is (misleadingly) suggested by the total regional trade share, and this dependence has remained virtually unchanged for the last decade. This imbalance in intra-regional trade is largely a reflection of the unique nature of Japan's involvement in fragmentation trade trade in East Asia. As already noted, Japan's trade relations with the rest of East Asia is predominantly in the form of using the region as an assembly base for meeting demand in the region and, more importantly for exporting to the rest of the world. As can be seen in Table 8 Japan has persistently maintained a trade surplus with all East Asian countries in both total manufacturing trade and trade in component, of which the latter is much larger.

Secondly, the measured degree of intra-regional trade integration needs to be qualified for the growing importance of cross border trade in components. The growth dynamism of such trade depends primarily on extra-regional demand. Moreover, component trade tends to infuse a presumably significant (though not measurable) bias into the conventionally measured intra-regional trade ratio; when components cross borders several time at different stages of production, the officially recorded value of trade artificially rises with each crossing.

As can be seen in Table 6 component trade accounts for a significant and growing share of intra-regional trade in manufacturing in East Asia, both on export and import sides. Moreover, the share of components in intra-regional trade is much larger than the 
comparable figures for the region's extra regional trade. In 2000, components accounted for 40.2 per cent of intra-East Asian exports, compared to 32 in the region's total exports. Between 1992 and 2000, trade in components accounted for 53 per cent of growth in total imports and 46 per cent of growth in total exports in East Asia. The significant of component trade looms even larger for developing East Asia and for the ASEAN. At the individual-country level, cross-border component trade accounts for more than a half of total imports and exports in Singapore, Malaysia and the Philippines, and more than a third in Thailand (Table A-3). Korea and Taiwan are also involved in sizable cross border trade with the other countries in the region. For all East Asian countries, between 1992 and 2000 the share of components in both intra-regional exports and imports has increased at a much faster rate compared that in exports to and imports from countries outside the region.

Given these observed patterns of dependence on component exchange, it is only natural that the degree of intra-regional trade dependence in East Asia turns out to be significantly lower when we focus on data on 'final trade' (total manufacturing trade net of parts and components) (Table 5). The intra-regional share in final trade in 2000 was 48.3 per cent compared to 52.3 per cent of total trade. More importantly, the alternative intra-regional trade share based on final trade has remained virtually stagnant between 1992 and 2000, in sharp contrast to the persistent increase recorded by the conventional measure. While this difference is observable for both exports and imports, the magnitude of the difference is much larger on the export side.

The difference in magnitude between regional trade shares estimated in gross and net terms is much larger for developing East Asia and ASEAN compared to estimates for the entire region. In 2000 over 60 per cent of 'final exports' from developing Asia found markets in countries outside the East Asian region, up from 55 per cent in 1992. A similar pattern is observable for the ASEAN countries. For all East Asian countries Japan is a much smaller market for net exports, accounting for less than 10 per cent in all cases, compared to the USA and the EU. It is also interesting to note that, unlike in the case of East Asia (or developing East Asia and the ASEAN), the estimated intra-regional 
trade share for NAFTA and the EU are remarkably resilient to the inclusion or exclusion of component trade.

In sum, the estimates presented in this section support our hypothesis that, in a context where fragmentation based trade in expanding rapidly, the standard trade flows analysis can lead to misleading inferences regarding the on-going process of economic integration through trade. When data on assembly trade are excluded from trade flows, our estimates suggest that extra-regional trade is much more important than intra-regional trade for continued growth dynamism of East Asia, both including and excluding Japan. Thus, the ongoing process of product fragmentation seems to have strengthened the case for a global, rather than a regional, approach to trade and investment policymaking.

\section{Conclusion and Inferences}

There is clear evidence that fragmentation trade is expanding more rapidly than conventional final-good trade. The degree of dependence on this new form of international specialisation is proportionately larger in East Asia compared to North America and Europe. This seems to be the outcome of the relatively more favourable policy setting for international production, agglomeration benefits arising from the early entry into this new form of specialisation, and considerable inter-country wage differential in the region.

A notable recent development in international product fragmentation in the region has been the rapid integration of China into the regional production networks. This development is an important counterpoint to the popular belief that China's global integration would crowd out opportunities for export-led industrialisation for other countries in the region.

The evidence harnessed in this paper on intra-regional patterns of trade flows has implications for the on-going debate on forming regional trading blocks within the region. Fragmentation trade has played a pivotal role in continuing dynamism of the East Asian economies and increasing intra-regional economic interdependence. But this new 
form of international specialisation could not be sustained purely as an East Asian phenomenon because of the growing importance of extra-regional markets for final goods. Put simply, growing trade in components has made the East Asia region increasingly reliant on extra-regional trade for its growth dynamism. In this context, these countries would be better off by upholding universal principles of economic openness. The Asia Pacific Economic Cooperation (APEC) agenda is certainly preferable to any East Asian regional grouping including or excluding Japan. But, in terms of opportunities for trade expansion through international product fragmentation, the ideal policy choice appears to be multilateral liberalisation through the WTO process. The ongoing process of product fragmentation seems to have strengthened the case for a global, rather than a regional, approach to trade and investment policymaking. 


\section{References}

Athukorala, Prema-chandra and Jayant Menon (1997), 'AFTA and the Investment-Trade Nexus in ASEAN', World Economy, 20(10), 159-174.

Baldwin, Robert E. (2001), 'The Implications of Increasing Fragmentation and Globalization for the World Trade Organization' in Leonard K. Cheng and Henryk Kierzkowski (eds.), Global Production and Trade in East Asia, Boston: Kluwer Academic Publishers, 249-271.

Barry, F. and J. Bradley (1997), 'FDI and Trade: The Irish Host Country Experience', Economic Journal, 107: 1798-811.

Borrus, Michael (1997), 'Left for Dead: Asian production Networks and the Revival of US Electronics', in Barry Naughton (ed), The China Circle: Economics and Technology in the PRC, Taiwan and Hong Kong, Washington DC: Brookings Institution Press.

Borrus, Michael (1999), 'Exploiting Asia to Beat Japan', in Dennis Encarnation, Japanese Multinationals in Asia, New York: Oxford University Press.

Brown, Drusilla K., Deardorff, Alan V and Robert M Stern (2003), 'The Effect of Multinational Production on Wages, and Working Conditions in Developing Countries, National Bureau of Economic Research (NBER) Working Paper, Cambridge, MA: NBER.

Cantwell, John (1994), 'The Relationship between International Trade and International Production' in David Greenway and L. Alan Winters (eds.), Survey in International Trade, Oxford: Basil Blackwell, pp. 303-328.

Crosby, N. and Y. Nakamori (1991), 'Motorola's Business Strategy in Southeast Asia', Journal of South East Asian Business, 7(1), 63-69.

Deardorff, Alan V. (2001), 'Financial Crisis, Trade, and Fragmentation', in Leonard K. Cheng and Henryk Kierzkowski (eds.), Global Production and Trade in East Asia, Boston: Kluwer Academic Publishers, 9-31.

Dobson, Wendy and Chia S.Yue (1997), Multinationals and East Asian Integration, Singapore: Institute of Southeast Asian Studies.

Drysdale, Peter and Ross Garnaut (1997), 'The Pacific: An Application of a General Theory of Economic Integration', in C. Fred Bergsten and Marcus Noland (eds), Pacific Dynamism and the International Economic System, Washington DC: Institute for International Economics, 183-224. 
Frankel, Jefrey A. and Shang-Jin Wei (1997), ' The New Regionalism and Asia: Impact and Policy Options', in Aravind Panagariya, M.G. Quibria and Narhari Rao (eds), The Global Trading System and Developing Asia, Oxford: oxford University Press, 83-130.

Freenstra, Robert (1998), 'Integration of Trade and Disintegration of production in the Global Economy', Journal of economic Perspectives, 14(4): 31-50.

Frobel, Kolket, Jurgan Heinrichs and Otto Kreye (1980), The International Division of Labour: Structural Unemployment in Industrialised Countries and Industrialisation in Developing Countries, Cambridge: Cambridge University Press.

Gereffi, Gary and T. Tam (1997), 'Commodity Chain and Regional Division of labour in East Asia', Journal of Asian Business, 12(1), 75-112.

Gereffi, Gary (1999), 'International Trade and Industrial Upgrading in the Apparel Commodity Chain', Journal of International Economics, 48(1), 37-70.

Hanson, Gordon H., Raymond J. Mataloni Jr., and Mathew J. Slaughter (2001), 'Expansion Strategires of U.S. Multinational Firms', in Susan M. Collins and Dani Rodrik (eds), Brookings Trade Forum 2001, Washington DC: Brookings Institution Press, 245-282.

Helleiner, Gerald K.(1973) 'Manufacturing Exports from Less Developed Countries and Multinational Firms', Economic Journal, 83: 21-47.

Hummels, David, Jun Ishii and Kei-Mu Yi (2001), 'The nature and growth of Vertical Specialization in World Trade', Journal of International Economics, 54(1), 75-96.

Irwin, Gouglas A. (2002), Free Trade Under Fire, Princeton, NJ: Princeton University Press.

Jones, Ronald W. (2000), Globalization and the Theory of Input Trade, Cambridge, Mass.: MIT Press.

Jones, Ronald W. and Henryk Kierzkowski (1990), 'The Role of Services in Production and International Trade: A Theoretical Framework', in Ronald W. Jones and Anne O Krueger (eds), The Political Economy of International Trade: Essays in Honor of Robert Baldwin, Oxfords: Basil Blackwell, 31-48.

Jones, Ronald W. and Henryk Kierzkowski (2001a), 'A Framework for Fragmentation', in Seven W. Arndt and Henryk Kierzkowski (eds), Fragmentation: New Production Patterns in the World Economy, New York: Oxford University Press, 17-34. 
Jones, Ronald W. and Henryk Kierzkowski (2001b), 'Horizontal Aspects of Vertical Fragmentation' in Leonard K. Cheng and Henryk Kierzkowski (eds.), Global Production and Trade in East Asia, Boston: Kluwer Academic Publishers, 33-51.

Jones, Ronald W. and Henryk Kierzkowski (2001c), 'Globalization and the Consequences of International Fragmentation', in Rudigar Dornbusch and G. Calvo and M. Obstfeld (eds), Money, Factor Mobility and Trade: The Festschrift in Honor of Robert A. Mundell, Cambridge, Mass.: MIT Press, 365-381.

Jones, Ronald W. and Henryk Kierzkowski and Gregory Leonard (2002), 'Fragmentation and Intra-Industry Trade', in Peter J. Lloyd and Hyun-Hoon Lee (eds.), Frontiers of Research in Intra-Industry Trade, Houndmills, UK: Palgrave, 67-86.

Kawai Masahiro and Shujiro Urata (1999), 'Are Trade and Direct Investment Substitutes or Complements? An Empirical Analysis of Japanese Manufacturing Industries', in Hiro Lee and David W. Roland-Holst (eds), Economic development and Cooperation in the Pacific basin: Trade, Investment, and Environmental Issues, Cambridge: Cambridge University Press, 251-296.

Kwai Masahiro and Shujiro Urata (2002), 'Trade and Foreign Direct Investment in East Asia', paper presented at the conference on Linkages in East asia: Implications for Currency Regimes and Policy Dialogue', Seoul, September 23-24, 2002.

Kwan, C.H. (2001), Yen Bloc: Toward Economic Integration in Asia, Washington DC: Brookings Institution Press.

Kierzkowski, Henryk (2001), 'Joining the Global Economy: Experience and prospects of the Transition Economies', Seven W. Arndt and Henryk Kierzkowski (eds), Fragmentation: New Production Patterns in the World Economy, New York: Oxford University Press, 231-253.

Krugman, Paul (1995), 'Growing World Trade: Causes and Consequences', Brooking Papers on Economic Activity, 25th Anniversary Issue: 327-77.

McKendrick, David G., Richard F. Doner and Stephen Haggard (2000), From Silicon Valley to Singapore: Location and Competitive Advantage in the Hard Disk Drive Industry, Stanford, Cal: Stanford University Press.

Naughton, Barry (ed), The China Circle: Economics and Technology in the PRC, Taiwan and Hong Kong, Washington DC: Brookings Institution Press.

Naughton, Barry (1999), 'The Global Electronics Revolution and China's Technology Policy', NBR Analysis, 10(2), 5-28.

Ng, Francis and Alexander Yeats (2001), 'Production Sharing in East Asia: Who does what for whom, and Why?' in Leonard K. Cheng and Henryk Kierzkowski (eds.), 
Global Production and Trade in East Asia, Boston: Kluwer Academic Publishers, 63-109.

Petri, Peter (1993), 'The East Asian Trading Block: An Analytical History', in J. Frankel and M. Kahler (eds), Regionalism and Rivalry: Japan and the United State in Pacific Asia, Chicago: Chicago University Press, 21-48.

Rangan, Subramanian and Robert Z. Lawrence (1999), A Prism on Globalization, Washington DC: Brookings Institution Press.

Ruane, Frances and Holger Gorg (2001), 'Globalization and Fragmentation: Evidence for the Electronics Industry in Ireland', in Seven W. Arndt and Henryk Kierzkowski (eds), Fragmentation: New Production Patterns in the World Economy, New York: Oxford University Press, 144-164.

Sharpton, Michael (1975), 'International Subcontracting', Oxford economic Papers, 27(1), 94-135.

Slaughter, Mathew J. (2000), 'Production Transfer within Multinational Enterprises and American Wages', Journal of International Economics, 50(3), 449-472.

Urata, Shujiro (2000), 'Emergence of an FDI-Trade Nexus and Economic Growth in East Asia', in Joseph E. Stiglitz and Hsaid Yususf (eds), Rethinking the East Asian Miracle, New York: Oxford University Press, 409-459.

USITC (United State International Trade Commission) (1999), Production Sharing: Use of U.S. Components and Material in Foreign Assembly Operations, 1995-1998, USITC Publication 3265, Washington DC: USITC.

Venable, Anthony J. (1999), 'Fragmentation and Multinational Production', European Economic Review, 43(3): 935-945.

Yeats, Alexander (2001), 'Just How Big is Global Production Sharing?', in Seven W. Arndt and Henryk Kierzkowski (eds), Fragmentation: New Production Patterns in the World Economy, New York: Oxford University Press, 108-143.

Zeile, William J. (1997), 'U.S. Intra-firm Trade in Goods', Survey of Current Business, February, 23-38. 
Table 1: World Trade in Parts and Components, 1992-2002 (\%)

\begin{tabular}{|c|c|c|c|c|c|c|c|c|c|}
\hline & Exports & & & Imports & & & Trade ba & nce & \\
\hline & 1992 & 1996 & 2000 & 1992 & 1995 & 2000 & 1992 & 1996 & 2000 \\
\hline East Asia & 34.5 & 38.3 & 39.5 & 33.5 & 32.8 & 33.1 & 24.1 & 11.0 & 13.4 \\
\hline Developing East Asia & 16.6 & 22.8 & 26.8 & 14.7 & 28.0 & 28.4 & -34.8 & -28.1 & -9.4 \\
\hline ASEAN & 7.7 & 11.8 & 13.6 & 12.6 & 14.5 & 12.0 & -37.4 & -27.8 & 8.5 \\
\hline Japan & 17.9 & 15.5 & 12.6 & 4.5 & 4.7 & 4.7 & 78.7 & 68.4 & 61.9 \\
\hline Indonesia & 0.2 & 0.3 & 0.5 & 1.2 & 0.9 & 0.3 & -525.2 & -259.3 & 26.1 \\
\hline Malaysia & 2.6 & 3.4 & 3.7 & 3.5 & 3.8 & 3.6 & -13.0 & -16.0 & 0.4 \\
\hline Philippines & 0.4 & 1.2 & 2.1 & 0.7 & 1.5 & 1.2 & -38.4 & -24.0 & 39.0 \\
\hline Singapore & 3.4 & 5.6 & 5.5 & 5.0 & 6.0 & 5.1 & -25.2 & -10.3 & 3.8 \\
\hline Thailand & 1.1 & 1.2 & 1.7 & 2.2 & 2.3 & 1.6 & -60.3 & -89.5 & 4.4 \\
\hline Vietnam & 0.0 & 0.0 & 0.1 & 0.0 & 0.1 & 0.1 & -1275.0 & -371.7 & -155.6 \\
\hline China & 1.1 & 1.7 & 3.0 & 3.5 & 2.9 & 4.9 & -168.5 & -78.5 & -71.4 \\
\hline Hong Kong & 2.2 & 0.9 & 0.5 & 3.3 & 4.6 & 4.9 & -29.8 & -408.9 & -837.0 \\
\hline Korea, RP & 3.0 & 3.8 & 4.3 & 3.8 & 3.3 & 3.3 & -9.5 & 10.5 & 20.3 \\
\hline Taiwan & 2.7 & 4.5 & 5.4 & 3.3 & 2.8 & 3.2 & -4.4 & 36.0 & 38.7 \\
\hline South Asia & 0.2 & 0.2 & 0.1 & 0.7 & 0.4 & 0.5 & -172.0 & -146.3 & -230.2 \\
\hline Ocean & 0.0 & 0.4 & 0.4 & 0.2 & 1.3 & 1.0 & -355.1 & -218.5 & -198.1 \\
\hline NAFTA & 28.2 & 24.0 & 23.9 & 33.5 & 25.8 & 27.5 & -0.1 & -11.8 & -18.9 \\
\hline USA & 22.1 & 18.7 & 17.9 & 23.2 & 17.7 & 17.7 & 11.8 & 1.7 & -1.9 \\
\hline Canada & 4.2 & 3.3 & 3.1 & 7.7 & 5.2 & 5.1 & -53.9 & -61.2 & -65.9 \\
\hline Mexico & 1.8 & 1.9 & 2.8 & 2.6 & 2.9 & 4.7 & -20.8 & -58.2 & -74.4 \\
\hline Europe & 46.6 & 53.9 & 42.5 & 51.4 & 49.7 & 36.1 & 7.2 & 4.2 & 12.4 \\
\hline $\mathrm{EU}$ & 32.8 & 38.0 & 30.9 & 35.1 & 33.8 & 21.5 & 10.0 & 7.6 & 28.3 \\
\hline Latin America & 0.6 & 0.6 & 2.1 & 1.3 & 2.2 & 3.7 & -75.2 & -255.8 & -81.2 \\
\hline Middle East & 0.0 & 0.5 & 0.7 & 2.3 & 1.2 & 0.8 & -6793.6 & -166.3 & -5.8 \\
\hline Africa & 0.1 & 0.2 & 0.1 & 0.4 & 0.9 & 0.4 & -479.2 & -319.4 & -236.9 \\
\hline World & 100 & 100 & 100 & 100 & 100 & 100 & & & \\
\hline World (\$ billion) & 336.8 & 756.9 & 1086.4 & 336.8 & 756.9 & 1086.4 & & & \\
\hline \multicolumn{10}{|l|}{ Memorandum Item } \\
\hline $\begin{array}{l}\text { Share of parts and components in } \\
\text { world manufacturing trade }(\%)\end{array}$ & 20.7 & \multicolumn{2}{|c|}{21.7} & 25.4 & 21.7 & 21.4 & 24.5 & & \\
\hline
\end{tabular}

Source: Compiled from UN Comtrade database. 
Table 2: Annual Compensation ${ }^{1}$ per Worker in Manufacturing, 1990-1998 (\$ per month)

\begin{tabular}{|l|r|r|r|r|r|r|r|r|r|}
\hline & 1990 & 1991 & 1992 & 1993 & 1994 & 1995 & 1996 & 1997 & 1998 \\
\hline All countries & 2257 & 2408 & 2529 & 2518 & 2560 & 2652 & 2693 & 2578 & 2539 \\
\hline Canada & 3156 & 3343 & 3350 & 3432 & 3589 & 3553 & 3570 & 3506 & 3413 \\
\hline Europe & 3074 & 3283 & 360 & 3388 & 3503 & 3724 & 3728 & 3616 & 3618 \\
\hline Latin America & 903 & 920 & 1009 & 1146 & 1175 & 1147 & 1253 & 1343 & 1284 \\
\hline Mexico & 579 & 666 & 765 & 843 & 854 & 749 & 746 & 831 & --- \\
\hline East Asia & 1788 & 1950 & 1963 & 2141 & 2129 & 2115 & 2138 & 1883 & 1787 \\
\hline China & 178 & 217 & 213 & 317 & 293 & 395 & 428 & 513 & 533 \\
\hline Hong Kong & 774 & 866 & 855 & n.a. & 850 & 844 & 1219 & 1077 & 1178 \\
\hline India & 348 & 315 & 323 & 335 & 318 & 427 & 463 & 461 & 474 \\
\hline Indonesia & 388 & 409 & 436 & 414 & 483 & 466 & 475 & 459 & 395 \\
\hline Japan & 3808 & 4108 & 4373 & 4902 & 5723 & 5934 & 5849 & 5343 & 5054 \\
\hline Korea & 1134 & 1449 & 1346 & 1635 & 1743 & 2059 & 2313 & 2351 & 2007 \\
\hline Malaysia & 387 & 420 & 488 & 570 & 573 & 569 & 665 & 657 & 652 \\
\hline Philippines & 314 & 370 & 467 & 499 & 565 & 580 & 647 & 623 & 598 \\
\hline Singapore* & 810 & 911 & 1067 & 1264 & 1477 & 1424 & 1623 & 1469 & 1720 \\
\hline Taiwan & 1162 & 1337 & 1639 & 1638 & 1695 & 1914 & n.a. & 2032 & 1970 \\
\hline Thailand & 343 & 428 & 502 & 569 & 508 & 523 & 568 & 508 & 472 \\
\hline Oceania & 1982 & 2142 & --- & 2304 & --- & --- & 3082 & 3024 & 2907 \\
\hline Australia & 2331 & 2446 & 2412 & 2377 & 2517 & 2694 & 2520 & 2400 & 2334 \\
\hline New Zealand & 1633 & 1838 & --- & 2230 &.--- & --- & 3645 & 3647 & 3480 \\
\hline Not & & & & & & & & &
\end{tabular}

Notes:

(1) Salary/wage plus other remuneration (including superannuation contribution) paid by foreign affiliates of U.S. MNEs.

(2) Covers only workers in industrial machinery sector.

--- Data suppressed in the original source for confidentiality.

Source: Compiled from, Survey of U.S. Direct Foreign Investment Abroad, Bureau of Economic Analysis, Department of Commerce, Washington DC (various issues). 
Table 3: Percentage Composition of Parts and Components Exports and Imports by three-digit ISIC Categories ${ }^{1}, 2000$

\section{(A) Exports}

\begin{tabular}{|c|c|c|c|c|c|c|c|c|c|c|c|c|c|c|c|c|}
\hline SITC & Description & $\mathrm{CHN}$ & HKG & KOR & IND & MAL & PHL & SGP & THA & $\mathrm{JPN}$ & EAS-J & EAS & NAF & USA & EU & World \\
\hline & Machinery and equipment & 91.9 & 84.1 & 98.1 & 93.9 & 98.6 & 99.5 & 98.5 & 96.9 & 95.0 & 97.0 & 96.3 & 94.8 & 90.8 & 93.6 & 94.2 \\
\hline 713 & Internal combustion engines & 0.8 & 0.3 & 0.4 & 0.8 & 0.1 & 0.2 & 0.4 & 0.6 & 4.2 & 0.4 & 1.8 & 3.7 & 4.7 & 3.1 & 2.6 \\
\hline 714 & Engines and motors, non-electric & 0.2 & 0.0 & 0.3 & 0.0 & 0.1 & 0.0 & 0.1 & 0.1 & 0.9 & 0.1 & 0.4 & 3.0 & 0.7 & 3.9 & 2.6 \\
\hline 716 & Rotating electrical engine & 0.8 & 1.1 & 0.3 & 0.4 & 0.1 & 0.0 & 0.2 & 0.4 & 0.7 & 0.3 & 0.4 & 0.5 & 1.2 & 0.6 & 0.6 \\
\hline 723 & Civil engineering/constructors' equipment & 0.6 & 0.1 & 0.5 & 0.7 & 0.1 & 0.0 & 1.5 & 0.1 & 0.4 & 0.6 & 0.5 & 0.9 & 0.9 & 1.3 & 1.3 \\
\hline 724 & Textile and leather machinery & 0.6 & 0.2 & 0.4 & 0.1 & 0.0 & 0.0 & 0.1 & 0.0 & 0.4 & 0.2 & 0.3 & 0.1 & 0.1 & 0.8 & 0.5 \\
\hline 728 & Specialised industrial machinery & 0.4 & 0.4 & 0.8 & 0.4 & 0.6 & 0.3 & 0.9 & 0.1 & 1.4 & 0.6 & 0.9 & 2.0 & 0.2 & 2.4 & 1.8 \\
\hline 735 & Parts and accessories for office machinery & 0.4 & 0.1 & 0.1 & 0.0 & 0.1 & 0.1 & 0.1 & 0.0 & 0.6 & 0.1 & 0.3 & 0.7 & 0.1 & 0.6 & 0.6 \\
\hline 737 & Metalworking machinery & 0.3 & 0.2 & 0.1 & 0.1 & 0.0 & 0.0 & 0.1 & 0.0 & 0.3 & 0.1 & 0.2 & 0.4 & 0.1 & 0.6 & 0.5 \\
\hline 741 & Heating and cooling equipment & 0.8 & 0.2 & 0.5 & 1.2 & 0.2 & 0.2 & 0.3 & 0.9 & 0.7 & 0.4 & 0.6 & 1.0 & 2.1 & 1.5 & 1.1 \\
\hline 742 & Pumps for liquid elevators & 0.3 & 0.0 & 0.1 & 0.2 & 0.0 & 0.0 & 0.1 & 0.0 & 0.3 & 0.1 & 0.2 & 0.5 & 0.2 & 0.8 & 0.5 \\
\hline 744 & Mechanical handling equipment & 0.5 & 0.1 & 0.2 & 0.2 & 0.1 & 0.0 & 0.1 & 0.1 & 0.4 & 0.2 & 0.3 & 1.2 & 0.5 & 1.2 & 0.8 \\
\hline 745 & Non-electrical machine tools and apparatus & 0.3 & 0.2 & 0.1 & 0.4 & 0.0 & 0.0 & 0.1 & 0.0 & 0.2 & 0.1 & 0.2 & 0.5 & 0.5 & 1.3 & 0.7 \\
\hline 749 & Non-electrical parts of machinery & 0.2 & 0.1 & 0.1 & 0.1 & 0.0 & 0.1 & 0.3 & 0.0 & 0.3 & 0.1 & 0.2 & 0.1 & 0.4 & 0.9 & 0.5 \\
\hline 759 & Parts and accessories for office machinery & 20.3 & 13.0 & 22.1 & 20.6 & 34.3 & 11.3 & 19.5 & 37.1 & 12.6 & 23.2 & 19.2 & 9.3 & 11.3 & 10.8 & 13.6 \\
\hline 764 & Telecommunication equipment, n.e.s & 18.2 & 8.8 & 7.5 & 27.3 & 4.9 & 2.0 & 5.1 & 5.7 & 7.5 & 7.6 & 7.6 & 12.8 & 10.4 & 7.1 & 7.4 \\
\hline 771 & Electrical power machinery & 1.2 & 4.0 & 0.5 & 0.2 & 0.2 & 0.0 & 0.3 & 0.8 & 0.4 & 0.6 & 0.5 & 0.2 & 0.7 & 0.4 & 0.5 \\
\hline 772 & Apparatus for switching/protecting electrical circuits & 11.3 & 6.9 & 3.1 & 9.7 & 6.6 & 7.3 & 5.7 & 10.7 & 10.0 & 6.8 & 8.0 & 4.4 & 17.4 & 9.0 & 8.4 \\
\hline 776 & Household electrical equipment, n.e.s & 17.9 & 44.0 & 54.4 & 15.3 & 48.4 & 74.9 & 59.7 & 33.4 & 31.8 & 49.1 & 42.6 & 10.2 & 10.4 & 15.2 & 26.1 \\
\hline 778 & Thermionic, cold cathodes or photo cathode valves and tubes & 6.1 & 2.9 & 1.0 & 7.8 & 1.0 & 0.1 & 1.2 & 1.4 & 4.7 & 1.9 & 2.9 & 0.9 & 7.1 & 2.5 & 2.7 \\
\hline 784 & Electrical machinery/apparatus, n.e.s & 3.7 & 0.0 & 3.9 & 4.8 & 0.4 & 2.6 & 0.8 & 2.9 & 13.0 & 2.2 & 6.2 & 32.2 & 19.6 & 17.8 & 13.6 \\
\hline 785 & Motorcycles and cycles & 2.2 & 0.0 & 0.1 & 2.6 & 0.3 & 0.1 & 0.5 & 1.3 & 1.1 & 0.7 & 0.8 & 0.1 & 0.1 & 0.5 & 0.5 \\
\hline 792 & Aircrafts and associated equipment & 1.2 & 0.0 & 0.4 & 0.4 & 0.5 & 0.1 & 0.9 & 0.3 & 1.1 & 0.6 & 0.8 & 4.8 & 0.3 & 5.4 & 3.9 \\
\hline & Other & 2.2 & 1.6 & 1.1 & 0.8 & 0.4 & 0.1 & 0.4 & 0.6 & 1.8 & 0.8 & 1.2 & 4.3 & 1.6 & 5.0 & 3.0 \\
\hline & Miscellaneous manufactured articles & 8.1 & 15.9 & 1.9 & 6.1 & 1.4 & 0.5 & 1.5 & 3.1 & 5.0 & 3.0 & 3.7 & 5.2 & 9.2 & 6.4 & 5.8 \\
\hline 821 & Furniture and parts thereof & 1.0 & 0.0 & 0.2 & 1.8 & 0.3 & 0.2 & 0.0 & 0.4 & 0.2 & 0.3 & 0.3 & 3.6 & 7.1 & 1.5 & 1.4 \\
\hline
\end{tabular}




\begin{tabular}{|c|c|c|c|c|c|c|c|c|c|c|c|c|c|c|c|c|}
\hline 846 & Clothing accessories of textile fabrics & 0.4 & 6.8 & 0.0 & 0.1 & 0.0 & 0.0 & 0.0 & 0.0 & 0.0 & 0.3 & 0.2 & 0.0 & 0.1 & 0.1 & 0.6 \\
\hline 851 & Footwear & 1.3 & 0.0 & 0.8 & 1.4 & 0.0 & 0.0 & 0.0 & 0.3 & 0.0 & 0.4 & 0.3 & 0.0 & 0.3 & 0.5 & 0.7 \\
\hline 874 & Measuring, analysing and checking equipment & 0.9 & 0.5 & 0.2 & 0.1 & 0.5 & 0.1 & 0.9 & 0.1 & 2.2 & 0.5 & 1.1 & 0.8 & 0.8 & 1.9 & 1.7 \\
\hline 881 & Photographic equipment, n.e.s & 0.4 & 4.1 & 0.1 & 0.5 & 0.2 & 0.1 & 0.2 & 0.9 & 0.6 & 0.4 & 0.5 & 0.0 & 0.1 & 0.2 & 0.6 \\
\hline \multirow[t]{2}{*}{891} & Arms and ammunition & 0.0 & 0.0 & 0.1 & 0.0 & 0.0 & 0.0 & 0.0 & 0.0 & 0.1 & 0.0 & 0.1 & 0.2 & 0.0 & 0.4 & 0.5 \\
\hline & Other & 4.0 & 4.5 & 0.5 & 2.2 & 0.5 & 0.1 & 0.3 & 1.4 & 1.8 & 1.1 & 1.4 & 0.5 & 0.9 & 1.8 & 0.3 \\
\hline & & & & & & & & & & & & & & & & \\
\hline \multirow{4}{*}{$7+8$} & Total & 100 & 100 & 100 & 100 & 100 & 100 & 100 & 100 & 100 & 100 & 100 & 100 & 100 & 100 & 100 \\
\hline & & & & & & & & & & & & & & & & \\
\hline & & & & & & & & & & & & & & & & \\
\hline & & $\mathrm{CHI}$ & $\mathrm{HOK}$ & Korea & INDI & MAL & Phil & SPR & Thai & Japan & EAS-J & EAS & NAF & USA & EU & World \\
\hline \multirow{2}{*}{$\frac{\text { SITC }}{7}$} & Description & & & & & & & & & & & & & & & \\
\hline & Machinery and equipment & 96.0 & 94.0 & 96.2 & 93.8 & 97.7 & 97.9 & 97.4 & 97.2 & 93.1 & 96.3 & 95.8 & 94.6 & 93.2 & 93.5 & 94.3 \\
\hline 713 & Internal combustion engines & 1.1 & 0.6 & 1.3 & 7.8 & 0.4 & 0.3 & 0.7 & 2.4 & 0.8 & 1.2 & 0.9 & 4.5 & 4.0 & 2.9 & 2.5 \\
\hline 714 & Engines and motors, non-electric & 0.2 & 1.4 & 0.5 & 0.2 & 0.3 & 0.8 & 0.6 & 1.0 & 3.8 & 0.8 & 1.1 & 2.9 & 2.0 & 3.6 & 2.4 \\
\hline 716 & Rotating electrical engine & 1.1 & 1.1 & 0.3 & 0.6 & 0.3 & 0.6 & 0.4 & 0.6 & 0.4 & 0.8 & 0.6 & 0.3 & 0.9 & 0.5 & 0.6 \\
\hline 723 & Civil engineering/constructors' equipment & 0.8 & 0.3 & 0.2 & 7.5 & 0.2 & 0.3 & 2.0 & 0.2 & 0.4 & 1.0 & 0.7 & 1.1 & 1.6 & 1.1 & 1.0 \\
\hline 728 & Specialised industrial machinery & 0.9 & 0.6 & 1.5 & 3.4 & 1.4 & 2.5 & 1.7 & 0.3 & 1.3 & 1.5 & 1.2 & 1.5 & 1.5 & 1.6 & 1.4 \\
\hline 735 & Parts and accessories for office machinery & 0.3 & 0.1 & 0.5 & 0.4 & 0.4 & 0.1 & 0.5 & 0.1 & 0.7 & 0.4 & 0.4 & 0.5 & 0.3 & 0.6 & 0.5 \\
\hline 741 & Heating and cooling equipment & 0.5 & 0.4 & 0.3 & 2.3 & 0.5 & 0.4 & 0.4 & 0.4 & 0.6 & 0.6 & 0.5 & 1.8 & 1.6 & 1.3 & 1.0 \\
\hline 742 & Pumps for liquid elevators & 0.2 & 0.1 & 0.2 & 2.3 & 0.1 & 0.1 & 0.3 & 0.1 & 0.2 & 0.2 & 0.2 & 0.7 & 0.6 & 0.7 & 0.5 \\
\hline 744 & Mechanical handling equipment & 0.3 & 0.3 & 0.2 & 0.8 & 0.1 & 0.1 & 0.3 & 0.2 & 0.3 & 0.3 & 0.3 & 0.7 & 0.9 & 1.0 & 0.7 \\
\hline 745 & Non-electrical machine tools and apparatus & 0.2 & 0.2 & 0.2 & 0.9 & 0.1 & 0.2 & 0.2 & 0.1 & 0.4 & 0.2 & 0.2 & 0.7 & 1.2 & 1.0 & 0.6 \\
\hline 747 & Taps cocks, valves and similar apparatus & 0.2 & 0.1 & 0.3 & 0.4 & 0.1 & 0.1 & 0.2 & 0.1 & 0.3 & 0.2 & 0.2 & 0.6 & 0.6 & 0.5 & 0.4 \\
\hline 759 & Parts and accessories for office machinery & 11.2 & 20.6 & 10.3 & 1.1 & 9.0 & 16.2 & 17.8 & 17.9 & 18.7 & 17.7 & 15.2 & 7.0 & 15.6 & 15.2 & 14.1 \\
\hline 764 & Telecommunication equipment, n.e.s & 14.9 & 13.6 & 6.5 & 2.5 & 6.1 & 9.5 & 4.8 & 3.6 & 9.7 & 11.1 & 9.2 & 4.8 & 10.7 & 7.2 & 7.7 \\
\hline 771 & Electrical power machinery & 1.0 & 2.0 & 0.5 & 0.2 & 0.9 & 0.6 & 0.4 & 0.4 & 0.3 & 1.1 & 0.8 & 0.1 & 0.1 & 0.3 & 0.5 \\
\hline 772 & Apparatus for switching/protecting electrical circuits & 10.2 & 8.1 & 6.5 & 5.6 & 9.5 & 5.2 & 6.6 & 10.5 & 5.9 & 10.0 & 7.8 & 7.5 & 6.8 & 8.1 & 8.6 \\
\hline 775 & Electro-diagnostic apparatus & 0.3 & 0.6 & 0.1 & 0.2 & 0.2 & 0.0 & 0.1 & 0.1 & 0.2 & 0.3 & 0.2 & 0.2 & 0.2 & 0.3 & 0.3 \\
\hline
\end{tabular}




\begin{tabular}{|c|c|c|c|c|c|c|c|c|c|c|c|c|c|c|c|c|}
\hline 776 & Household electrical equipment, n.e.s & 39.6 & 37.9 & 57.0 & 2.4 & 63.5 & 55.8 & 55.1 & 48.0 & 38.9 & 59.9 & 47.4 & 15.0 & 8.5 & 17.8 & 28.1 \\
\hline 778 & Thermionic, cold cathodes or photo cathode valves ect. & 3.0 & 3.2 & 2.5 & 3.2 & 1.6 & 1.7 & 1.0 & 1.3 & 1.3 & 2.7 & 2.0 & 1.6 & 2.8 & 2.7 & 2.5 \\
\hline 784 & Electrical machinery/apparatus, n.e.s & 4.0 & 0.8 & 3.4 & 30.9 & 0.8 & 1.8 & 1.2 & 7.7 & 4.0 & 3.3 & 2.9 & 33.4 & 14.5 & 16.9 & 13.3 \\
\hline 785 & Motorcycles and cycles & 0.4 & 0.5 & 0.1 & 7.4 & 0.2 & 0.2 & 0.3 & 0.4 & 0.5 & 0.5 & 0.4 & 0.3 & 0.5 & 0.8 & 0.6 \\
\hline 792 & Aircrafts and associated equipment & 1.0 & 0.3 & 1.5 & 3.9 & 0.6 & 0.1 & 1.4 & 0.0 & 2.1 & 1.1 & 1.1 & 4.6 & 11.5 & 4.2 & 2.9 \\
\hline & Other & 4.1 & 0.9 & 1.9 & 7.6 & 1.3 & 1.2 & 1.3 & 1.4 & 2.0 & -19.1 & 1.9 & 4.5 & 6.5 & 4.9 & 3.7 \\
\hline & & & & & & & & & & & & & & & & \\
\hline & Miscellaneous manufactured articles & 4.0 & 6.0 & 3.8 & 6.2 & 2.3 & 2.1 & 2.6 & 2.8 & 6.9 & 3.7 & 4.2 & 5.4 & 6.8 & 6.5 & 5.7 \\
\hline 821 & Furniture and parts thereof & 0.2 & 0.2 & 0.1 & 0.2 & 0.2 & 0.1 & 0.1 & 0.1 & 1.3 & 0.2 & 0.3 & 2.7 & 0.9 & 1.8 & 1.5 \\
\hline 846 & Clothing accessories of textile fabrics & 0.1 & 0.8 & 0.0 & 0.1 & 0.0 & 0.1 & 0.0 & 0.0 & 0.0 & 0.3 & 0.2 & 0.0 & 0.0 & 0.0 & 0.6 \\
\hline 848 & Clothing accessories other than textile fabrics & 0.0 & 0.0 & 0.0 & 0.0 & 0.0 & 0.0 & 0.0 & 0.0 & 0.0 & 0.0 & 0.0 & 0.0 & 0.0 & 0.0 & 0.5 \\
\hline 851 & Footwear & 0.5 & 0.6 & 0.3 & 2.3 & 0.0 & 0.1 & 0.0 & 0.2 & 0.4 & 0.4 & 0.3 & 0.1 & 0.4 & 0.6 & 0.5 \\
\hline 874 & Measuring, analysing and checking equipment & 0.8 & 0.7 & 1.5 & 0.9 & 1.4 & 0.2 & 1.7 & 0.8 & 2.8 & 1.3 & 1.4 & 1.5 & 2.4 & 1.8 & 1.5 \\
\hline 881 & Photographic equipment, n.e.s & 1.2 & 1.4 & 0.3 & 0.3 & 0.4 & 0.7 & 0.3 & 0.3 & 0.5 & 0.9 & 0.7 & 0.1 & 0.2 & 0.2 & 0.5 \\
\hline & Other & 1.1 & 2.2 & 1.6 & 2.4 & 0.3 & 0.9 & 0.4 & 1.4 & 1.8 & 0.6 & 1.3 & 1.0 & 2.8 & 2.1 & 0.5 \\
\hline & & & & & & & & & & & & & & & & \\
\hline $7+8$ & Total & 100 & 100 & 100 & 100 & 100 & 100 & 100 & 100 & 100 & 100 & 100 & 100 & 100 & 100 & 100 \\
\hline
\end{tabular}

Notes:

1 5-digit SITC parts and components items aggregated at the 3-digit level 2. Including Mexico (MEX).

2 Excluding Mexico.

Source: Compiled from UN Comtrade database. 
Table 4: Share of Parts and Components in Manufacturing Trade

\section{(A) Exports}

\begin{tabular}{|c|c|c|c|c|c|}
\hline \multirow[t]{2}{*}{ Country/region } & \multicolumn{3}{|c|}{$\begin{array}{l}\text { Share of parts and } \\
\text { Components }(\%)\end{array}$} & \multirow{2}{*}{\begin{tabular}{|l|}
$\begin{array}{l}\text { Export } \\
\text { growth (\%) }\end{array}$ \\
$1992-2000$ \\
\end{tabular}} & \multirow{2}{*}{\begin{tabular}{|l} 
Contribution of parts \\
and components to \\
export growth (\%)
\end{tabular}} \\
\hline & 1992 & 1996 & 2000 & & \\
\hline East Asia & 21.3 & 28.0 & 32.0 & 3.8 & $\begin{array}{ll}83.5 \\
8\end{array}$ \\
\hline Developing East Asia & 19.7 & 26.7 & 32.8 & 5.1 & 41.9 \\
\hline ASEAN & 26.4 & 35.0 & 44.4 & 5.6 & 55.0 \\
\hline Japan & 22.9 & 30.2 & 30.6 & 1.8 & 50.4 \\
\hline Indonesia & 4.0 & 7.4 & 14.2 & 4.4 & 22.7 \\
\hline Malaysia & 40.4 & 42.6 & 49.7 & 6.2 & 54.3 \\
\hline Philippines & 23.9 & 52.5 & 64.0 & 9.0 & 74.3 \\
\hline Singapore & 28.2 & 39.7 & 49.6 & 4.9 & 64.5 \\
\hline Thailand & 21.2 & 23.4 & 35.9 & 4.8 & 46.7 \\
\hline Vietnam & 2.0 & 5.2 & 8.7 & 21.0 & 8.9 \\
\hline China & 6.7 & 9.8 & 14.5 & 6.7 & 17.9 \\
\hline Hong Kong & 21.5 & 26.7 & 25.8 & -3.2 & 16.3 \\
\hline Korea, RP & 17.8 & 25.2 & 30.6 & 4.5 & 41.0 \\
\hline Taiwan & 20.1 & 28.8 & 37.8 & 5.8 & 47.4 \\
\hline South Asia & 4.7 & 4.9 & 0.7 & 14.4 & 0.3 \\
\hline \begin{tabular}{|l|} 
Oceania \\
\end{tabular} & 6.4 & 18.9 & 15.2 & 12.6 & 16.3 \\
\hline NAFTA & 26.2 & 27.2 & 28.1 & 4.1 & 30.0 \\
\hline USA & 26.9 & 30.5 & 31.6 & 3.3 & 37.5 \\
\hline Canada & 19.4 & 19.7 & 18.0 & 4.2 & 16.8 \\
\hline Mexico & 21.5 & 19.4 & 21.1 & 8.1 & 21.0 \\
\hline \begin{tabular}{|l|} 
Europe \\
\end{tabular} & 13.9 & 16.2 & 17.5 & 3.6 & 21.4 \\
\hline EU & 15.5 & 17.7 & 18.9 & 3.9 & 22.2 \\
\hline Latin America & 8.6 & 11.7 & 10.2 & 11.6 & 10.5 \\
\hline \begin{tabular}{|l|} 
Middle East \\
\end{tabular} & 3.0 & 13.6 & 18.9 & 13.9 & 20.5 \\
\hline Africa & 6.9 & 8.0 & 8.6 & 8.0 & 9.2 \\
\hline World & 20.7 & 21.7 & 25.4 & 4.2 & 29.5 \\
\hline
\end{tabular}


(B) Imports

\begin{tabular}{|c|c|c|c|c|c|}
\hline \multirow[t]{2}{*}{ Country/region } & \multicolumn{3}{|c|}{$\begin{array}{l}\text { Share of parts and } \\
\text { Components }(\%)\end{array}$} & \multirow{2}{*}{\begin{tabular}{|l} 
Import \\
growth (\%) \\
$1992-00$ \\
\end{tabular}} & \multirow{2}{*}{$\begin{array}{l}\text { Contribution of parts } \\
\text { and components to } \\
\text { export growth (\%) }\end{array}$} \\
\hline & 1992 & 1996 & 2000 & & \\
\hline East Asia & 22.8 & 27.9 & 35.4 & 4.4 & 45.9 \\
\hline East Asia-Japan & 24.8 & 30.2 & 38.4 & 4.5 & 49.3 \\
\hline ASEAN & 30.4 & 39.3 & 48.6 & 3.6 & 68.2 \\
\hline Japan & 15.4 & 19.3 & 24.2 & 4.1 & 32.1 \\
\hline Indonesia & 20.5 & 23.8 & 19.4 & -0.1 & 63.6 \\
\hline Malaysia & 37.9 & 47.5 & 58.8 & 4.2 & 77.5 \\
\hline Philippines & 32.6 & 43.6 & 55.1 & 6.9 & 64.4 \\
\hline Singapore & 32.0 & 42.8 & 51.7 & 3.9 & 70.7 \\
\hline Thailand & 26.6 & 32.9 & 39.8 & 2.6 & 62.0 \\
\hline Vietnam & 4.2 & 11.1 & 19.1 & 10.1 & 22.1 \\
\hline China & 19.5 & 21.1 & 33.5 & 5.4 & 42.0 \\
\hline Hong Kong & 15.1 & 20.4 & 28.2 & 5.2 & 36.7 \\
\hline Korea, RP & 26.7 & 27.4 & 38.9 & 3.6 & 52.1 \\
\hline Taiwan & 29.6 & 35.0 & 37.3 & 5.1 & 42.5 \\
\hline South Asia & 14.0 & 14.6 & 7.2 & 7.9 & 5.0 \\
\hline Oceania & 10.4 & 15.2 & 15.6 & 12.9 & 16.2 \\
\hline NAFTA & 20.4 & 23.6 & 22.8 & 4.8 & 24.6 \\
\hline USA & 18.2 & 21.7 & 19.4 & 4.7 & 20.3 \\
\hline Canada & 24.6 & 28.7 & 26.3 & 3.8 & 28.0 \\
\hline Mexico & 19.1 & 30.6 & 30.7 & 7.3 & 35.1 \\
\hline Europe & 15.2 & 16.6 & 18.7 & 3.4 & 22.8 \\
\hline $\mathrm{EU}$ & 16.0 & 18.9 & 20.3 & 2.4 & 28.0 \\
\hline Latin America & 14.4 & 14.6 & 15.1 & 12.6 & 15.2 \\
\hline Middle East & 23.6 & 18.5 & 16.7 & 2.3 & 3.5 \\
\hline Africa & 11.8 & 14.5 & 10.7 & 6.7 & 10.2 \\
\hline World & 21.7 & 21.4 & 24.5 & 5.9 & 26.0 \\
\hline
\end{tabular}

\section{--- Data not available.}

Source: Compiled from UN Comtrade database. 
Table 5: Direction of Manufacturing Trade (\%)

\section{(A ) Trade (export + imports)}

\begin{tabular}{|c|c|c|c|c|c|c|c|c|c|c|c|c|c|c|c|c|c|c|c|}
\hline \multirow[t]{2}{*}{ Region/Country } & & \multicolumn{9}{|c|}{ A1: Total manufacturing trade } & \multicolumn{9}{|c|}{ B1: Total manufacturing trade net of parts and components } \\
\hline & & EAS & $\mathrm{JPN}$ & DEA & ASEAN & NAFTA & USA & EU & Other & $\begin{array}{l}\text { World (\$ } \\
\text { bn) }\end{array}$ & EAS & JPN & DEA & ASEAN & NAFTA & USA & EU & Other & $\begin{array}{l}\text { World } \\
\text { (\$bn) }\end{array}$ \\
\hline \multirow[t]{3}{*}{ East Asia (EAS) } & 1992 & 47.1 & 12.3 & 34.9 & 11.2 & 26.3 & 24.6 & 16.6 & 9.9 & 1110.3 & 45.3 & 11.5 & 33.8 & 9.3 & $\begin{array}{r}25.6 \\
\end{array}$ & 23.9 & 17.8 & \begin{tabular}{l|l|} 
& 11.4 \\
\end{tabular} & 867.3 \\
\hline & 1996 & 51.5 & 12.9 & 38.5 & 14.4 & 23.0 & 20.8 & 16.5 & 9.0 & 1883.1 & 49.2 & 12.0 & 37.2 & 11.7 & 22.0 & 20.0 & 17.9 & 10.9 & 1356.5 \\
\hline & 2000 & 52.3 & 12.0 & 40.2 & 14.4 & 23.7 & 21.9 & 15.3 & 8.7 & 2312.1 & 48.3 & 11.4 & 36.9 & 10.7 & 24.1 & 22.3 & 16.7 & 10.9 & 1536.9 \\
\hline \multirow[t]{3}{*}{ Japan (JPN) } & 1992 & 32.0 & 0.0 & 32.0 & 10.9 & 32.2 & 29.4 & 22.4 & 13.4 & 412.0 & 30.5 & 0.0 & 30.5 & 9.6 & 30.6 & 27.9 & 23.8 & 15.2 & 325.1 \\
\hline & 1996 & 41.4 & 0.0 & 41.4 & 16.3 & 30.2 & 28.3 & 18.3 & 10.1 & 560.0 & 39.5 & 0.0 & 39.5 & 14.3 & 28.4 & 26.7 & 20.0 & 12.1 & 411.3 \\
\hline & 2000 & 42.5 & 0.0 & 42.5 & 14.4 & 31.2 & 28.9 & 17.5 & 8.8 & 644.3 & 39.3 & 0.0 & 39.3 & 11.7 & 31.0 & 28.9 & 19.1 & 10.6 & 460.6 \\
\hline \multirow[t]{3}{*}{ Developing East Asia (DEA) } & 1992 & 56.1 & 19.6 & 36.5 & 11.3 & 22.8 & 21.8 & 13.2 & 7.9 & 698.4 & 54.1 & 18.4 & 35.8 & 9.1 & 22.5 & 21.5 & 14.2 & 9.1 & 542.2 \\
\hline & 1996 & 55.7 & 18.4 & 37.3 & 13.6 & 20.0 & 17.7 & 15.7 & 8.6 & 1323.0 & 53.4 & 17.2 & 36.2 & 10.6 & 19.2 & 17.1 & 16.9 & 10.4 & 945.2 \\
\hline & 2000 & 56.0 & 16.7 & 39.4 & 14.4 & 20.8 & 19.2 & 14.5 & 8.7 & 1667.8 & 52.2 & 16.3 & 35.9 & 10.3 & 21.1 & 19.5 & 15.7 & 11.1 & 1076.3 \\
\hline \multirow[t]{3}{*}{ ASEAN } & 1992 & 51.1 & 20.2 & 30.9 & 18.2 & 23.3 & 22.2 & 17.8 & 7.8 & 256.9 & 47.5 & 19.4 & 28.0 & 15.4 & 23.0 & 21.7 & 20.0 & 9.6 & 183.5 \\
\hline & 1996 & 55.2 & 19.2 & 36.0 & 20.7 & 20.7 & 19.9 & 17.1 & 7.0 & 525.4 & 51.3 & 18.8 & 32.5 & 16.7 & 20.4 & 19.6 & 19.4 & 8.9 & 329.5 \\
\hline & 2000 & 56.8 & 16.5 & 40.3 & 23.6 & 20.8 & 19.8 & 14.8 & 7.5 & 591.6 & 52.1 & 17.3 & 34.8 & 19.0 & 20.7 & 19.5 & 16.9 & 10.3 & 317.6 \\
\hline \multirow[t]{3}{*}{ NAFTA } & 1992 & 31.0 & 13.6 & 17.4 & 5.4 & 39.2 & 19.6 & 18.8 & 11.0 & 980.5 & 31.5 & 13.4 & 18.0 & 5.0 & 37.6 & 19.2 & 19.4 & 11.5 & 755.3 \\
\hline & 1996 & 30.0 & 11.3 & 18.7 & 6.8 & 43.1 & 21.9 & 16.3 & 10.6 & 1470.5 & 28.6 & 10.6 & 18.0 & 5.9 & 43.4 & 22.1 & 16.7 & 11.4 & 1100.4 \\
\hline & 2000 & 27.8 & 9.3 & 18.5 & 5.7 & 46.3 & 24.2 & 16.3 & 9.5 & 2202.4 & 26.2 & 8.8 & 17.4 & 4.4 & 46.4 & 24.1 & 17.1 & 10.3 & 1652.7 \\
\hline \multirow[t]{3}{*}{ USA } & 1992 & 38.3 & 16.7 & 21.7 & 6.9 & 25.8 & 0.0 & 22.4 & 13.4 & 730.6 & 38.7 & 16.3 & 22.3 & 6.3 & 24.3 & 0.0 & 23.0 & 14.1 & 564.0 \\
\hline & 1996 & 38.4 & 14.4 & 24.0 & 8.8 & 28.5 & 0.0 & 20.0 & 13.1 & 1066.2 & 36.7 & 13.6 & 23.1 & 7.7 & 28.7 & 0.0 & 20.5 & 14.1 & 795.7 \\
\hline & 2000 & 36.5 & 12.1 & 24.4 & 7.7 & 30.9 & 0.0 & 20.6 & 12.0 & 1532.9 & 34.4 & 11.6 & 22.8 & 5.9 & 31.1 & 0.0 & 21.6 & 12.9 & 1152.1 \\
\hline \multirow[t]{3}{*}{ European Union (EU) } & 1992 & 8.5 & 3.8 & 4.6 & 1.4 & 7.8 & 7.0 & 70.8 & 13.0 & 1773.2 & 8.6 & 3.9 & 4.7 & 1.4 & 7.3 & 6.6 & 71.2 & 12.9 & 1524.1 \\
\hline & 1996 & 11.3 & 3.7 & 7.6 & 2.9 & 9.1 & 8.2 & 61.0 & 18.7 & 2910.0 & 10.8 & 3.5 & 7.3 & 2.6 & 8.4 & 7.6 & 62.0 & 18.9 & 2377.6 \\
\hline & 2000 & 11.6 & 3.6 & 8.0 & 2.6 & 11.4 & 10.2 & 58.2 & 18.8 & 3310.0 & 10.8 & 3.4 & 7.4 & 2.1 & 10.9 & 9.8 & 59.6 & 18.7 & 2661.4 \\
\hline \multirow[t]{3}{*}{ World (WLD) } & 1992 & 28.7 & 9.7 & 18.9 & 6.2 & 26.2 & 18.1 & 32.8 & 12.4 & 2710.0 & 26.9 & 8.9 & 17.9 & 5.1 & 24.6 & 17.5 & 34.9 & 13.6 & 2114.3 \\
\hline & 1996 & 25.3 & 7.5 & 17.8 & 6.5 & 20.4 & 15.1 & 39.1 & 15.2 & 6900.0 & 23.3 & 6.8 & 16.5 & 5.3 & 19.3 & 14.2 & 41.1 & 16.3 & 5415.1 \\
\hline & 2000 & 25.7 & 7.0 & 18.8 & 6.2 & 23.8 & 17.1 & 36.1 & 14.4 & 8570.0 & 22.3 & 6.2 & 16.2 & 4.3 & 23.2 & 16.8 & 38.7 & 15.7 & 6430.8 \\
\hline
\end{tabular}




\begin{tabular}{|c|c|c|c|c|c|c|c|c|c|c|c|c|c|c|c|c|c|c|c|}
\hline \multicolumn{20}{|l|}{ (B) Exports } \\
\hline & & \multicolumn{9}{|c|}{ A2: Manufacturing exports } & \multicolumn{9}{|c|}{ B2: Manufacturing exports net of parts and components } \\
\hline & & EAS & JPN & DEA & ASEAN & NAF & USA & $\mathrm{EU}$ & Other & $\begin{array}{l}\begin{array}{l}\text { World (\$ } \\
\text { bn) }\end{array} \\
\end{array}$ & EAS & JPN & DEA & ASEAN & NAFTA & A USA & EU & Other & \begin{tabular}{|l|l}
$\begin{array}{l}\text { Wolrd } \\
\text { (\$bn) }\end{array}$ \\
\end{tabular} \\
\hline \multirow[t]{3}{*}{ East Asia (EAS) } & 1992 & 39.5 & 4.7 & 34.9 & 12.2 & 30.4 & 28.2 & 17.4 & t 12.7 & 649.5 & 5 & 4.8 & 32.8 & 10.1 & 29.7 & 27.6 & 18.4 & 14.4 & 4511.5 \\
\hline & 1996 & 45.7 & 7.2 & 38.5 & 16.4 & 26.6 & 23.4 & 15.7 & 12.0 & 994.3 & 43.2 & 7.7 & 35.5 & 13.1 & 25.9 & 23.1 & 16.4 & 14.5 & 715.7 \\
\hline & 2000 & 43.7 & 7.5 & 36.1 & 13.8 & 28.5 & 26.1 & 16.6 & 11.3 & 1297.3 & 38.4 & 7.6 & 30.8 & 9.9 & 29.9 & 27.6 & 17.5 & 14.2 & 881.9 \\
\hline \multirow[t]{3}{*}{ Japan (JPN) } & 1992 & 31.1 & 0.0 & 31.1 & 11.3 & 32.7 & 29.3 & 20.8 & 15.4 & 312.8 & 29.0 & 0.0 & 29.0 & 9.8 & 31.5 & 28.2 & 21.8 & 17.7 & 241.1 \\
\hline & 1996 & 40.7 & 0.0 & 40.7 & 17.3 & 30.8 & 28.6 & 16.2 & 12.4 & 374.7 & 38.0 & 0.0 & 38.0 & 14.7 & 29.8 & 27.9 & 17.0 & 15.2 & 261.7 \\
\hline & 2000 & 38.2 & 0.0 & 38.2 & 13.6 & 33.9 & 31.2 & 17.1 & 10.9 & 435.1 & 33.9 & 0.0 & 33.9 & 10.4 & 35.0 & 32.5 & 17.8 & 13.3 & 302.1 \\
\hline \multirow[t]{3}{*}{ East Asia excl. Japan (EAS-J) } & 1992 & 47.2 & 9.1 & 38.3 & 12.9 & 28.3 & 27.1 & 14.2 & 10.2 & 336.8 & 45.1 & 9.1 & 36.3 & 10.3 & 28.1 & 26.9 & 15.3 & 11.4 & 270.3 \\
\hline & 1996 & 48.8 & 11.6 & 37.2 & 15.9 & 24.0 & 20.3 & 15.4 & 11.8 & 619.7 & 46.1 & 12.1 & 34.1 & 12.2 & 23.6 & 20.3 & 16.1 & 14.1 & 454.0 \\
\hline & 2000 & 46.4 & 11.3 & 35.1 & 14.0 & 25.7 & 23.6 & 16.3 & 11.6 & 862.2 & 40.8 & 11.5 & 29.3 & 9.7 & 27.2 & 25.0 & 17.3 & 14.7 & 579.7 \\
\hline \multirow[t]{3}{*}{ ASEAN } & 1992 & 41.3 & 9.0 & 32.3 & 20.3 & 28.7 & 27.3 & 18.9 & 11.1 & 117.3 & 37.0 & 8.7 & 28.3 & 16.6 & 28.7 & 27.0 & 21.1 & 13.2 & 86.3 \\
\hline & 1996 & 50.6 & 11.4 & 39.2 & 24.5 & 24.0 & 22.8 & 16.8 & 8.6 & 245.9 & 46.1 & 12.5 & 33.7 & 19.1 & 24.9 & 23.6 & 18.1 & 10.9 & 159.9 \\
\hline & 2000 & 50.3 & 11.0 & 39.3 & 23.1 & 23.7 & 22.2 & 16.8 & 9.2 & 322.4 & 43.2 & 12.3 & 30.9 & 17.2 & 25.2 & 23.7 & 18.5 & 13.1 & 179.3 \\
\hline \multirow[t]{3}{*}{ NAFTA } & 1992 & 18.6 & 6.4 & 12.2 & 4.5 & 45.3 & 22.0 & 19.9 & 16.2 & 429.4 & 18.2 & 6.6 & 11.6 & 3.7 & 44.8 & 23.1 & 20.2 & 16.9 & 316.8 \\
\hline & 1996 & 20.2 & 6.6 & 13.6 & 5.5 & 49.0 & 26.4 & 15.7 & 15.1 & 643.1 & 18.5 & 6.6 & 11.9 & 4.2 & 50.4 & 29.1 & 15.3 & 15.8 & 468.4 \\
\hline & 2000 & 16.7 & 5.0 & 11.7 & 4.4 & 55.7 & 31.4 & 15.9 & 11.7 & 892.4 & 13.7 & 4.7 & 9.0 & 2.6 & 58.3 & 34.9 & 15.7 & 12.2 & 641.2 \\
\hline \multirow[t]{3}{*}{ USA } & 1992 & 23.9 & 8.2 & 15.7 & 5.8 & 30.9 & 0.0 & 25.0 & 20.2 & 320.5 & 23.7 & 8.6 & 15.1 & 4.8 & 29.2 & 0.0 & 25.7 & 21.4 & 231.9 \\
\hline & 1996 & 27.6 & 9.2 & 18.4 & 7.5 & 31.9 & 0.0 & 21.0 & 19.5 & 447.7 & 26.3 & 9.5 & 16.8 & 5.9 & 31.5 & 0.0 & 21.2 & 21.0 & 311.3 \\
\hline & 2000 & 24.6 & 7.4 & 17.2 & 6.6 & 36.7 & 0.0 & 22.5 & 16.2 & 582.9 & 21.4 & 7.4 & 14.0 & 4.1 & 37.3 & 0.0 & 23.6 & 17.7 & 394.4 \\
\hline \multirow[t]{3}{*}{ European Union (EU) } & 1992 & 4.5 & 1.4 & 3.2 & 1.4 & 7.1 & 5.9 & 69.5 & 18.9 & 836.7 & 4.4 & 1.4 & 3.0 & 1.3 & 6.6 & 5.6 & 70.2 & 18.9 & 705.7 \\
\hline & 1996 & 8.9 & 2.4 & 6.5 & 2.7 & 8.5 & 7.6 & 59.2 & 23.4 & 1560.0 & 8.6 & 2.5 & 6.1 & 2.4 & 8.2 & 7.3 & 60.0 & 23.2 & 1283.3 \\
\hline & 2000 & 7.1 & 2.0 & 5.2 & 1.8 & 11.6 & 10.2 & 58.7 & 22.6 & 1720.0 & 6.4 & 2.1 & 4.3 & 1.3 & 11.6 & 10.2 & 60.1 & 21.9 & 1394.8 \\
\hline \multirow[t]{3}{*}{ World (WLD) } & 1992 & 23.3 & 4.3 & 19.0 & 6.8 & 28.8 & 19.9 & 31.0 & 17.0 & 1360.0 & 21.4 & 4.2 & 17.2 & 5.4 & 27.1 & 19.8 & 32.9 & 18.6 & 1057.5 \\
\hline & 1996 & 20.3 & 4.3 & 16.1 & 6.8 & 21.2 & 15.9 & 38.4 & 20.1 & 3360.0 & 18.4 & 4.3 & 14.1 & 5.2 & 20.2 & 15.5 & 40.1 & 21.3 & 2632.0 \\
\hline & 2000 & 19.2 & 4.0 & 15.2 & 5.8 & 26.1 & 19.5 & 36.7 & 18.0 & 4140.0 & 15.1 & 3.7 & 11.4 & 3.6 & 26.0 & 20.3 & 39.4 & 19.5 & \begin{tabular}{|l|l}
5087.2 \\
5
\end{tabular} \\
\hline \multicolumn{20}{|l|}{ (C) Imports } \\
\hline & & & & $(\mathrm{A} 3$ & 3) $\mathrm{Ma}$ & anufact & turing it & mports & & & (B3) & Manu & ufacturi & ing impc & orts ne & et of par & rts and $\mathrm{c}$ & compor & nents \\
\hline
\end{tabular}




\begin{tabular}{|c|c|c|c|c|c|c|c|c|c|c|c|c|c|c|c|c|c|c|c|}
\hline & & EAS & JPN & DEA & ASEAN & NAF & USA & $\mathrm{EU}$ & Other & WLD & EAS & JPN & DEA & ASEAN & NAF & USA & $\mathrm{EU}$ & Other & WLD \\
\hline \multirow[t]{3}{*}{ East Asia (EAS) } & 1992 & 58.0 & 23.1 & 34.9 & 9.7 & 20.5 & 19.6 & 15.5 & 6.0 & 460.8 & 56.4 & 21.2 & 35.2 & 8.2 & 19.6 & 18.6 & 17.0 & 7.1 & 355.8 \\
\hline & 1996 & 57.9 & 19.3 & 38.6 & 12.1 & 19.0 & 17.9 & 17.4 & 5.7 & 888.7 & 56.0 & 16.9 & 39.1 & 10.2 & 17.7 & 16.7 & 19.5 & 6.9 & 640.8 \\
\hline & 2000 & 63.3 & 17.8 & 45.4 & 15.1 & 17.6 & 16.6 & 13.8 & 5.4 & 1014.8 & 61.6 & 16.5 & 45.1 & 11.8 & 16.2 & 15.2 & 15.6 & 6.5 & 655.1 \\
\hline \multirow[t]{3}{*}{ Japan (JPN) } & 1992 & 34.7 & 0.0 & 34.7 & 9.6 & 30.9 & 29.7 & 27.3 & 7.1 & 99.2 & 34.7 & 0.0 & 34.7 & 8.9 & 28.0 & 26.7 & 29.5 & 7.8 & 83.9 \\
\hline & 1996 & 42.9 & 0.0 & 42.9 & 14.2 & 29.0 & 27.7 & 22.5 & 5.6 & 185.4 & 42.2 & 0.0 & 42.2 & 13.5 & 26.1 & 24.7 & 25.2 & 6.5 & 149.6 \\
\hline & 2000 & 51.4 & 0.0 & 51.4 & 16.1 & 25.7 & 24.1 & 18.5 & 4.5 & 209.2 & 49.6 & 0.0 & 49.6 & 14.3 & 23.5 & 21.9 & 21.5 & 5.4 & 158.5 \\
\hline \multirow[t]{3}{*}{ East Asia excl. Japan (EAS-J) } & 1992 & 64.3 & 29.4 & 34.9 & 9.8 & 17.7 & 16.9 & 12.3 & 5.7 & 361.6 & 63.1 & 27.8 & 35.3 & 8.0 & 17.0 & 16.1 & 13.1 & 6.8 & 271.9 \\
\hline & 1996 & 61.8 & 24.4 & 37.5 & 11.6 & 16.4 & 15.3 & 16.1 & 5.7 & 703.4 & 60.2 & 22.0 & 38.1 & 9.2 & 15.2 & 14.2 & 17.7 & 7.0 & 491.2 \\
\hline & 2000 & 66.3 & 22.5 & 43.9 & 14.8 & 15.5 & 14.6 & 12.6 & 5.6 & 805.6 & 65.4 & 21.8 & 43.7 & 11.0 & 13.9 & 13.1 & 13.7 & 6.9 & 496.5 \\
\hline \multirow[t]{3}{*}{ ASEAN } & 1992 & 59.4 & 29.7 & 29.7 & 16.5 & 18.7 & 18.0 & 16.8 & 5.1 & 139.7 & 56.7 & 28.9 & 27.8 & 14.2 & 17.9 & 17.0 & 19.1 & 6.3 & 97.2 \\
\hline & 1996 & 59.2 & 26.0 & 33.2 & 17.3 & 17.9 & 17.4 & 17.4 & 5.5 & 279.5 & 56.2 & 24.9 & 31.3 & 14.4 & 16.3 & 15.8 & 20.5 & 7.0 & 169.6 \\
\hline & 2000 & 64.7 & 23.2 & 41.5 & 24.1 & 17.5 & 16.8 & 12.4 & 5.5 & 269.2 & 63.7 & 23.8 & 39.9 & 21.2 & 14.8 & 14.1 & 14.7 & 6.8 & 138.3 \\
\hline \multirow[t]{3}{*}{ NAFTA } & 1992 & 40.6 & 19.2 & 21.3 & 6.1 & 34.4 & 17.8 & 18.0 & 7.0 & 551.1 & 41.1 & 18.4 & 22.7 & 6.0 & 32.5 & 16.3 & 18.8 & 7.6 & 438.5 \\
\hline & 1996 & 37.7 & 14.9 & 22.8 & 7.8 & 38.5 & 18.5 & 16.7 & 7.1 & 827.4 & 36.0 & 13.6 & 22.4 & 7.2 & 38.2 & 16.9 & 17.7 & 8.1 & 632.0 \\
\hline & 2000 & 35.4 & 12.3 & 23.1 & 6.6 & 39.9 & 19.3 & 16.7 & 8.0 & 1310.0 & 34.2 & 11.4 & 22.7 & 5.5 & 38.8 & 17.2 & 17.9 & 9.1 & 1011.4 \\
\hline \multirow[t]{3}{*}{ USA } & 1992 & 49.6 & 23.3 & 26.4 & 7.8 & 21.8 & 0.0 & 20.4 & 8.1 & 410.2 & 49.1 & 21.7 & 27.4 & 7.4 & 20.8 & 0.0 & 21.1 & 8.9 & 332.1 \\
\hline & 1996 & 46.3 & 18.3 & 28.0 & 9.7 & 26.0 & 0.0 & 19.3 & 8.4 & 618.5 & 43.4 & 16.3 & 27.1 & 8.8 & 26.9 & 0.0 & 20.1 & 9.7 & 484.4 \\
\hline & 2000 & 43.8 & 15.1 & 28.7 & 8.3 & 27.3 & 0.0 & 19.5 & 9.4 & 949.9 & 41.2 & 13.8 & 27.4 & 6.8 & 27.8 & 0.0 & 20.6 & 10.4 & 757.7 \\
\hline \multirow[t]{3}{*}{ European Union (EU) } & 1992 & 12.0 & 6.1 & 5.9 & 1.5 & 8.4 & 8.0 & 71.9 & 7.6 & 936.5 & 12.2 & 6.0 & 6.2 & 1.6 & 7.8 & 7.4 & 72.1 & 7.8 & 818.4 \\
\hline & 1996 & 14.0 & 5.2 & 8.9 & 3.1 & 9.7 & 9.0 & 63.1 & 13.2 & 1350.0 & 13.4 & 4.7 & 8.7 & 2.8 & 8.5 & 7.9 & 64.3 & 13.8 & 1094.4 \\
\hline & 2000 & 16.5 & 5.3 & 11.2 & 3.4 & 11.2 & 10.3 & 57.6 & 14.7 & 1590.0 & 15.6 & 4.8 & 10.8 & 3.0 & 10.2 & 9.4 & 59.0 & 15.2 & 1266.6 \\
\hline \multirow[t]{3}{*}{ World (WLD) } & 1992 & 34.2 & 15.3 & 18.9 & 5.5 & 23.5 & 16.2 & 34.6 & 7.7 & 1350.0 & 32.4 & 13.6 & 18.7 & 4.8 & 22.0 & 15.3 & 37.0 & 8.6 & 1056.8 \\
\hline & 1996 & 29.9 & 10.6 & 19.4 & 6.2 & 19.7 & 14.3 & 39.8 & 10.5 & 3540.0 & 28.0 & 9.3 & 18.7 & 5.3 & 18.5 & 12.9 & 41.9 & 11.6 & 2783.1 \\
\hline & 2000 & 31.8 & 9.7 & 22.1 & 6.6 & 21.7 & 14.9 & 35.4 & 11.0 & 4430.0 & 29.0 & 8.4 & 20.5 & 5.0 & 20.6 & 13.6 & 38.1 & 12.3 & 3343.6 \\
\hline
\end{tabular}

Source: Complied from UN Comtrade Database using the commodity/country classification described in the text (Section 3). 
Table 6: Share of Parts and Component contents in Regional Manufacturing Trade Flows (\%)

\begin{tabular}{|c|c|c|c|c|c|c|c|c|c|c|c|c|c|c|c|c|c|}
\hline \multirow[t]{2}{*}{ Exporter/Importer } & & \multicolumn{8}{|l|}{ Exports } & \multicolumn{8}{|l|}{ Imports } \\
\hline & & EAS & JPN & DEA & ASEAN & NAF & USA & EUU & WLD & EAS & JPN & DEA & ASEAN & NAF & USA & EUU & WLD \\
\hline \multirow[t]{3}{*}{ East Asia (EA) } & 1992 & 25.2 & 20.2 & 25.8 & 34.9 & 23.1 & 22.9 & 16.8 & 21.3 & 24.9 & 29.0 & 22.1 & 35.1 & 26.5 & 26.9 & 15.6 & 22.8 \\
\hline & 1996 & 32.1 & 24.0 & 33.6 & 42.4 & 29.9 & 29.1 & 24.5 & 28.0 & 30.3 & 36.9 & 27.0 & 39.5 & 32.9 & 32.9 & 19.2 & 27.9 \\
\hline & 2000 & 40.2 & 31.3 & 42.0 & 51.3 & 28.6 & 28.3 & 28.2 & 32.0 & 37.1 & 40.3 & 35.9 & 49.6 & 40.4 & 40.7 & 26.7 & 35.4 \\
\hline \multirow[t]{3}{*}{ Japan (JPN) } & 1992 & 28.3 & --- & 28.3 & 33.4 & 25.6 & 25.7 & 19.3 & 22.9 & 15.4 & --- & 15.4 & 22.1 & 23.5 & 23.7 & 8.5 & 15.4 \\
\hline & 1996 & 34.9 & --- & 34.9 & 40.6 & 32.4 & 31.9 & 26.5 & 30.2 & 20.6 & --- & 20.6 & 23.2 & 27.6 & 28.1 & 9.4 & 19.3 \\
\hline & 2000 & 38.4 & --- & 38.4 & 46.8 & 28.2 & 27.5 & 27.6 & 30.6 & 26.8 & --- & 26.8 & 32.8 & 30.7 & 31.1 & 11.6 & 24.2 \\
\hline \multirow[t]{3}{*}{ East Asia excl. Japan (DEA) } & 1992 & 23.3 & 20.2 & 23.9 & 36.1 & 20.3 & 20.1 & 13.4 & 19.7 & 26.3 & 29.0 & 23.9 & 38.7 & 27.9 & 28.5 & 19.9 & 24.8 \\
\hline & 1996 & 30.7 & 24.0 & 32.8 & 43.7 & 27.9 & 26.8 & 23.3 & 26.7 & 32.0 & 36.9 & 28.9 & 44.7 & 35.4 & 35.2 & 22.8 & 30.2 \\
\hline & 2000 & 40.9 & 31.3 & 44.0 & 53.5 & 28.9 & 28.8 & 28.6 & 32.8 & 39.2 & 40.3 & 38.7 & 54.3 & 44.6 & 44.8 & 32.5 & 38.4 \\
\hline \multirow[t]{3}{*}{ ASEAN } & 1992 & 33.9 & 28.5 & 35.4 & 39.7 & 26.5 & 27.1 & 17.8 & 26.4 & 33.5 & 32.2 & 34.9 & 40.0 & 33.5 & 34.1 & 21.2 & 30.4 \\
\hline & 1996 & 40.7 & 29.1 & 44.1 & 49.3 & 32.6 & 32.7 & 29.7 & 35.0 & 42.4 & 41.9 & 42.8 & 49.5 & 44.7 & 45.0 & 28.6 & 39.3 \\
\hline & 2000 & 52.2 & 38.0 & 56.2 & 58.7 & 40.8 & 40.7 & 38.7 & 44.4 & 49.4 & 47.1 & 50.7 & 54.6 & 56.4 & 56.8 & 38.9 & 48.6 \\
\hline \multirow[t]{3}{*}{ NAFTA } & 1992 & 28.0 & 23.6 & 30.3 & 39.3 & 27.1 & 22.2 & 25.2 & 26.2 & 19.5 & 23.9 & 15.5 & 22.2 & 25.0 & 26.9 & 16.8 & 20.4 \\
\hline & 1996 & 33.1 & 27.4 & 35.9 & 45.0 & 25.0 & 19.7 & 29.3 & 27.2 & 26.9 & 30.4 & 24.6 & 29.5 & 24.3 & 30.1 & 19.3 & 23.6 \\
\hline & 2000 & 40.9 & 31.5 & 44.9 & 57.2 & 24.8 & 20.1 & 28.8 & 28.1 & 25.5 & 27.9 & 24.2 & 35.2 & 24.8 & 31.0 & 17.1 & 22.8 \\
\hline \multirow[t]{3}{*}{ USA } & 1992 & 28.3 & 24.0 & 30.6 & 40.1 & 31.6 & --- & 25.5 & 27.6 & 19.8 & 24.4 & 15.7 & 22.6 & 22.8 & --- & 16.4 & 19.0 \\
\hline & 1996 & 33.7 & 28.1 & 36.4 & 45.6 & 31.5 & --- & 29.7 & 30.5 & 26.6 & 30.1 & 24.4 & 29.3 & 18.9 & --- & 18.5 & 21.7 \\
\hline & 2000 & 41.1 & 31.9 & 45.1 & 57.6 & 31.1 & --- & 29.1 & 32.3 & 25.0 & 27.1 & 23.8 & 34.9 & 18.9 & --- & 15.9 & 20.2 \\
\hline \multirow[t]{3}{*}{ European Union (EU) } & 1992 & 18.4 & 11.9 & 21.1 & 21.8 & 21.1 & 20.3 & 14.8 & 15.6 & 10.7 & 13.3 & 8.0 & 9.1 & 18.9 & 19.3 & 12.4 & 12.6 \\
\hline & 1996 & 20.3 & 13.0 & 23.0 & 27.9 & 21.2 & 20.7 & 16.6 & 17.7 & 22.5 & 26.5 & 20.1 & 27.1 & 28.5 & 28.6 & 17.5 & 18.9 \\
\hline & 2000 & 27.1 & 14.6 & 31.9 & 38.7 & 19.0 & 18.4 & 17.0 & 18.9 & 24.4 & 27.8 & 22.9 & 29.8 & 27.8 & 27.5 & 18.4 & 20.3 \\
\hline \multirow[t]{3}{*}{ World (WLD) } & 1992 & 28.5 & 23.0 & 29.8 & 39.1 & 26.6 & 22.5 & 17.3 & 22.2 & 25.8 & 30.0 & 22.4 & 32.6 & 26.8 & 26.4 & 16.5 & 21.7 \\
\hline & 1996 & 29.3 & 21.2 & 31.5 & 39.7 & 25.2 & 23.4 & 18.1 & 21.7 & 26.5 & 31.1 & 24.0 & 32.6 & 26.3 & 29.1 & 17.2 & 21.4 \\
\hline & 2000 & 41.2 & 30.4 & 44.1 & 53.8 & 25.6 & 22.3 & 20.1 & 25.4 & 31.3 & 34.6 & 29.9 & 43.3 & 28.4 & 31.1 & 18.8 & 24.5 \\
\hline
\end{tabular}

Source: Complied from UN Comtrade Database using the commodity/country classification described in the text (Section 3). 
Table 6: The Growth of Manufacturing Trade and the Contribution of Trade in Parts and Components to Trade Growth (\%)

\begin{tabular}{|c|c|c|c|c|c|c|c|c|c|c|c|c|c|c|c|c|}
\hline \multirow[t]{2}{*}{ Exporter/Importer } & \multicolumn{8}{|c|}{ Export Growth } & \multicolumn{8}{|c|}{ Contribution of Parts and Components to Export Growth } \\
\hline & EA & $\mathrm{JPN}$ & DEA & ASEAN & $\mathrm{NAF}$ & USA & EU & World & EA & JPN & DEA & ASEAN & NAF & USA & EU & World \\
\hline East Asia (EA) & 4.4 & 6.5 & 4.0 & 4.6 & 3.5 & 3.4 & 3.6 & 3.8 & 52.6 & 36.4 & 57.1 & 64.2 & 35.0 & 34.5 & 40.9 & 42.8 \\
\hline Japan (JPN) & 2.9 & --- & 2.9 & 2.8 & 2.0 & 2.1 & 0.7 & 1.8 & 52.7 & --- & 52.7 & 66.9 & 34.1 & 31.2 & 86.2 & 50.1 \\
\hline East Asia excl. Japan (DEA) & 5.1 & 6.5 & 4.7 & 5.7 & 4.7 & 4.4 & 6.0 & 5.2 & 52.5 & 36.4 & 58.9 & 63.4 & 35.3 & 35.8 & 36.4 & 41.1 \\
\hline ASEAN & 6.8 & 6.8 & 6.8 & 6.4 & 4.5 & 4.5 & 5.0 & 5.6 & 60.0 & 42.1 & 65.1 & 67.6 & 52.1 & 51.6 & 53.1 & 54.7 \\
\hline NAFTA & 3.4 & 2.7 & 3.8 & 4.0 & 5.2 & 6.1 & 2.8 & 4.1 & 55.8 & 44.1 & 59.6 & 74.4 & 23.3 & 19.0 & 34.2 & 29.9 \\
\hline USA & 3.5 & 2.7 & 3.8 & 4.0 & 4.3 & --- & 2.7 & 3.3 & 55.9 & 44.5 & 59.6 & 73.9 & 30.7 & --- & 34.8 & 38.1 \\
\hline EU & 6.6 & 6.0 & 6.8 & 5.5 & 6.8 & 7.1 & 3.0 & 4.0 & 31.1 & 16.0 & 36.5 & 48.8 & 18.1 & 17.7 & 20.0 & 22.0 \\
\hline \multirow[t]{3}{*}{ World } & 5.1 & 5.8 & 5.0 & 5.3 & 5.7 & 6.1 & 7.2 & 6.2 & 49.6 & 34.4 & 54.0 & 63.0 & 25.1 & 22.2 & 21.1 & 27.0 \\
\hline & \multicolumn{8}{|c|}{ Import Growth } & \multicolumn{8}{|c|}{ Contribution of Parts and Components to Import Growth } \\
\hline & EA & JAP & DEA & ASEAN & NAF & USA & EU & World & EA & JAP & DEA & ASEAN & NAF & USA & EU & World \\
\hline East Asia (EA) & 4.9 & 2.9 & 5.9 & 6.9 & 3.5 & 3.4 & 3.7 & 4.4 & 45.9 & 56.3 & 43.3 & 55.6 & 56.2 & 56.6 & 38.5 & 46.0 \\
\hline Japan (JPN) & 6.4 & --- & 6.4 & 7.1 & 3.1 & 3.0 & 1.9 & 4.1 & 32.2 & --- & 32.2 & 37.0 & 40.3 & 41.4 & 18.9 & 32.2 \\
\hline East Asia excl. Japan (DEA) & 4.6 & 2.9 & 5.7 & 6.8 & 3.7 & 3.6 & 4.5 & 4.4 & 49.2 & 56.3 & 46.8 & 60.9 & 62.3 & 62.3 & 42.5 & 49.4 \\
\hline ASEAN & 4.1 & 2.2 & 5.5 & 5.8 & 3.2 & 3.3 & 1.9 & 3.6 & 63.8 & 76.9 & 60.0 & 62.7 & 85.0 & 85.0 & 81.2 & 68.2 \\
\hline NAFTA & 4.0 & 2.3 & 5.3 & 5.2 & 5.7 & 5.3 & 4.4 & 4.8 & 31.1 & 35.8 & 29.7 & 43.6 & 24.7 & 33.6 & 17.3 & 24.5 \\
\hline USA & 4.0 & 2.2 & 5.2 & 5.0 & 5.9 & --- & 4.4 & 4.7 & 29.9 & 32.5 & 29.1 & 43.2 & 16.8 & --- & 15.4 & 21.1 \\
\hline $\mathrm{EU}$ & 4.7 & 2.2 & 6.5 & 7.6 & 4.5 & 4.3 & 1.7 & 2.9 & 34.8 & 57.7 & 29.6 & 37.1 & 34.9 & 34.4 & 35.1 & 31.4 \\
\hline World & 6.3 & 4.1 & 7.6 & 7.7 & 6.2 & 6.1 & 6.8 & 6.7 & 34.0 & 38.8 & 32.5 & 46.9 & 29.1 & 33.5 & 19.8 & 25.8 \\
\hline
\end{tabular}

Source: Complied from UN Comtrade Database using the commodity/country classification described in the text (Section 3). 
Table 8: Bilateral Trade Balances (as a percentage of exports)

\section{(A) Trade in total manufacturing}

\begin{tabular}{|c|c|c|c|c|c|c|c|c|c|}
\hline \multirow[t]{2}{*}{ Region/country } & \multicolumn{9}{|c|}{ Trading partner region/country } \\
\hline & & EA & JPN & DEA & ASEAN & NAFTA & USA & EU & WLD \\
\hline \multirow[t]{3}{*}{ ASEAN } & 1992 & -41.7 & -74.6 & -8.8 & 3.5 & 29.1 & 27.6 & -5.8 & -16.1 \\
\hline & 1996 & -24.8 & -61.3 & 3.8 & 24.8 & 18.0 & 15.1 & -15.5 & -12.0 \\
\hline & 2000 & -6.8 & -43.0 & 13.4 & 15.2 & 62.6 & 58.5 & 62.5 & 19.8 \\
\hline \multirow[t]{3}{*}{ East Asia (EA) } & 1992 & -4.0 & -71.1 & 40.9 & 76.1 & 108.7 & 102.1 & 57.8 & 41.0 \\
\hline & 1996 & -11.6 & -57.9 & 11.6 & 51.5 & 56.1 & 46.4 & 0.9 & 11.9 \\
\hline & 2000 & -11.8 & -46.2 & 1.7 & 17.6 & 107.1 & 101.2 & 53.7 & 27.8 \\
\hline \multirow[t]{3}{*}{ Japan (JPN) } & 1992 & 182.6 & --- & 182.6 & 272.3 & 233.0 & 211.7 & 140.7 & 215.3 \\
\hline & 1996 & 91.7 & --- & 91.7 & 145.3 & 114.2 & 108.6 & 45.5 & 102.1 \\
\hline & 2000 & 54.6 & --- & 54.6 & 75.6 & 174.5 & 168.6 & 92.7 & 108.0 \\
\hline \multirow[t]{3}{*}{ Developing East Asia (DEA) } & 1992 & -31.6 & -71.1 & 2.2 & 23.2 & 49.1 & 49.3 & 7.4 & -6.9 \\
\hline & 1996 & -30.5 & -57.9 & -12.6 & 21.2 & 29.1 & 16.8 & -15.6 & -11.9 \\
\hline & 2000 & -25.1 & -46.2 & -14.3 & 1.2 & 78.0 & 72.3 & 38.9 & 7.0 \\
\hline \multirow[t]{3}{*}{ NAFTA } & 1992 & -64.3 & -74.2 & -55.3 & -42.8 & 2.4 & -3.7 & -13.8 & -22.1 \\
\hline & 1996 & -58.4 & -65.5 & -53.7 & -45.0 & -1.2 & 10.7 & -26.9 & -22.3 \\
\hline & 2000 & -67.9 & -72.4 & -65.4 & -54.2 & -4.8 & 11.0 & -35.2 & -31.9 \\
\hline \multirow[t]{3}{*}{ USA } & 1992 & -62.3 & -72.4 & -53.4 & -41.7 & 10.5 & --- & -4.6 & -21.9 \\
\hline & 1996 & -56.9 & -63.7 & -52.5 & -43.9 & -11.0 & --- & -21.4 & -27.6 \\
\hline & 2000 & -65.5 & -70.1 & -63.2 & -51.3 & -17.7 & --- & -29.2 & -38.6 \\
\hline \multirow[t]{3}{*}{ European Union (EU) } & 1992 & -66.3 & -79.9 & -52.3 & -20.2 & -25.3 & -34.2 & -13.7 & -10.7 \\
\hline & 1996 & -26.9 & -46.4 & -15.5 & -0.8 & 1.8 & -2.9 & 8.3 & 15.6 \\
\hline & 2000 & -53.3 & -60.2 & -50.0 & -44.6 & 12.2 & 6.5 & 10.2 & 8.2 \\
\hline
\end{tabular}

(B) Trade in Parts and components

\begin{tabular}{|c|c|c|c|c|c|c|c|c|c|}
\hline & & EA & JPN & DEA & ASEAN & NAFTA & USA & EU & WLD \\
\hline \multirow[t]{3}{*}{ ASEAN } & 1992 & -69.7 & -345.2 & -8.1 & 2.8 & 1.9 & 1.5 & -26.0 & -37.4 \\
\hline & 1996 & -38.3 & -271.7 & 6.6 & 19.7 & -16.2 & -19.4 & -13.8 & -27.8 \\
\hline & 2000 & -1.5 & -117.5 & 20.5 & 19.2 & 15.0 & 12.0 & 38.1 & 8.5 \\
\hline \multirow[t]{3}{*}{ East Asia (EA) } & 1992 & -2.7 & -394.9 & 39.1 & 42.8 & 45.0 & 41.9 & 41.3 & 24.0 \\
\hline & 1996 & -6.7 & -265.2 & 28.1 & 38.6 & 29.4 & 22.9 & 22.3 & 11.0 \\
\hline & 2000 & -4.8 & -139.0 & 16.0 & 17.9 & 31.8 & 28.5 & 38.4 & 13.4 \\
\hline \multirow[t]{3}{*}{ Japan (JPN) } & 1992 & 80.7 & --- & 80.7 & 82.3 & 72.4 & 70.4 & 81.7 & 78.7 \\
\hline & 1996 & 69.1 & --- & 69.1 & 76.7 & 60.2 & 57.8 & 75.6 & 68.4 \\
\hline & 2000 & 54.8 & --- & 54.8 & 60.1 & 60.4 & 57.9 & 78.2 & 61.9 \\
\hline \multirow[t]{3}{*}{ Developing East Asia (DEA) } & 1992 & -64.6 & -394.9 & 2.1 & 13.1 & 8.1 & 5.2 & -37.8 & -35.1 \\
\hline & 1996 & -50.1 & -265.2 & -0.8 & 15.4 & 1.8 & -12.2 & -16.1 & -28.1 \\
\hline & 2000 & -28.0 & -139.0 & -2.6 & -0.3 & 13.2 & 9.7 & 18.0 & -9.4 \\
\hline \multirow[t]{3}{*}{ NAFTA } & 1992 & -94.6 & -292.1 & -14.4 & 1.0 & 9.7 & -25.4 & 23.0 & -0.1 \\
\hline & 1996 & -95.1 & -221.9 & -47.9 & -19.3 & 1.6 & -38.1 & 10.0 & -11.8 \\
\hline & 2000 & -93.9 & -222.1 & -55.8 & -34.5 & -5.2 & -39.1 & 8.6 & -18.9 \\
\hline \multirow[t]{2}{*}{ USA } & 1992 & -85.5 & -268.5 & -10.5 & 3.2 & 34.7 & --- & 32.6 & 11.8 \\
\hline & 1996 & -83.6 & -194.6 & -41.0 & -14.5 & 32.7 & --- & 20.6 & 1.7 \\
\hline
\end{tabular}




\begin{tabular}{|l|l|r|r|r|r|r|r|r|r|}
\hline & 2000 & -76.1 & -184.1 & -43.4 & -24.4 & 26.4 & --- & 23.1 & -1.9 \\
\hline European Union (EU) & 1992 & -72.6 & -452.8 & 20.2 & 47.7 & -20.0 & -44.2 & 3.0 & 9.8 \\
\hline & 1996 & -51.2 & -280.9 & -3.3 & 2.0 & -32.3 & -42.4 & 2.6 & 7.6 \\
\hline & 2000 & -92.6 & -377.7 & -43.2 & -39.0 & -30.7 & -40.1 & 1.7 & 0.5 \\
\hline
\end{tabular}

(C) Trade in manufacturing net of parts and components

\begin{tabular}{|c|c|c|c|c|c|c|c|c|c|}
\hline & & EA & JPN & DEA & ASEAN & NAFTA & USA & EU & WLD \\
\hline \multirow[t]{3}{*}{ ASEAN } & 1992 & -72.5 & -273.0 & -10.6 & 3.8 & 30.0 & 29.1 & -1.8 & -12.6 \\
\hline & 1996 & -29.2 & -111.5 & 1.3 & 20.1 & 30.5 & 29.0 & -20.2 & -6.1 \\
\hline & 2000 & -13.7 & -49.8 & 0.6 & 4.7 & 54.7 & 54.0 & 38.7 & 22.9 \\
\hline \multirow[t]{3}{*}{ East Asia (EA) } & 1992 & -4.6 & -207.6 & 25.5 & 43.4 & 54.2 & 53.1 & 35.7 & 30.4 \\
\hline & 1996 & -16.1 & -97.5 & 1.4 & 30.6 & 38.8 & 35.3 & -6.1 & 10.5 \\
\hline & 2000 & -19.1 & -61.6 & -8.7 & 11.9 & 59.7 & 58.9 & 33.6 & 25.7 \\
\hline \multirow[t]{3}{*}{ Japan (JPN) } & 1992 & 58.3 & --- & 58.3 & 68.6 & 69.1 & 67.1 & 52.9 & 65.2 \\
\hline & 1996 & 36.4 & --- & 36.4 & 47.3 & 50.0 & 49.4 & 15.3 & 42.8 \\
\hline & 2000 & 23.2 & --- & 23.2 & 28.0 & 64.8 & 64.6 & 36.6 & 47.5 \\
\hline \multirow[t]{3}{*}{ Developing East Asia (DEA) } & 1992 & -40.6 & -207.6 & -2.2 & 22.0 & 39.3 & 40.0 & 13.8 & -0.6 \\
\hline & 1996 & -41.0 & -97.5 & -21.0 & 19.1 & 30.6 & 24.2 & -19.2 & -8.2 \\
\hline & 2000 & -37.4 & -61.6 & -27.8 & 2.8 & 56.3 & 55.0 & 32.0 & 14.4 \\
\hline \multirow[t]{3}{*}{ NAFTA } & 1992 & -212.9 & -286.5 & -171.1 & -124.0 & -0.4 & 2.3 & -29.1 & -38.4 \\
\hline & 1996 & -162.5 & -177.7 & -154.1 & -133.1 & -2.1 & 21.4 & -56.3 & -34.9 \\
\hline & 2000 & -292.1 & -281.5 & -297.7 & -230.6 & -5.0 & 22.3 & -79.6 & -57.7 \\
\hline \multirow[t]{3}{*}{ USA } & 1992 & -196.9 & -260.3 & -160.6 & -121.8 & -2.1 & --- & -17.6 & -43.2 \\
\hline & 1996 & -156.7 & -168.2 & -150.2 & -131.8 & -33.0 & --- & -47.3 & -55.6 \\
\hline & 2000 & -269.9 & -257.7 & -276.4 & -215.2 & -43.2 & --- & -67.7 & -92.1 \\
\hline \multirow[t]{3}{*}{ European Union (EU) } & 1992 & -224.2 & -388.9 & -144.6 & -45.6 & -37.6 & -54.0 & -19.2 & -16.0 \\
\hline & 1996 & -33.1 & -57.7 & -22.7 & -1.9 & 11.0 & 7.3 & 8.7 & 14.7 \\
\hline & 2000 & -121.9 & -112.3 & -126.5 & -106.8 & 20.6 & 16.5 & 10.8 & 9.2 \\
\hline
\end{tabular}

Source: Complied from UN Comtrade Database using the commodity/country classification described in the text (Section 3). 


\section{Statistical Appendix}

\section{Contents}

Table A-1: $\quad$ Share of Parts and Components in Bilateral Manufacturing Trade Flows, 1992, 1996 and 2000

Table A-2: $\quad$ Growth of Bilateral Manufacturing Trade Flow and the Contribution Parts and Components to Trade growth, 19922000

Table A-3: $\quad$ Direction of Manufacturing Trade, 1992, 1996 and 2000 (percentage shares)

Table A-4: $\quad$ Bilateral Trade Balances, 1992, 1996, 2000 (as a percentage of exports)

Table A-5: $\quad$ List of Parts and Components Identified at the Five-digit Level of the Standard International Classification (SITC) System (Revision 3) 
Table A-1: East Asian: Direction of Manufacturing Trade, 1992, 1996 and 2000 (percentage shares)

\section{(A) Manufacturing Exports}

\begin{tabular}{|c|c|c|c|c|c|c|c|c|c|c|c|c|c|c|c|c|c|c|c|c|}
\hline & & $\mathrm{CHN}$ & HKG & IDN & KOR & MAL & PHL & SGP & THA & TWN & VNM & JPN & ASEAN & EAS & EAS-J & $\mathrm{NAF}$ & USA & $\mathrm{EU}$ & Other & $\begin{array}{l}\text { WLD } \\
\text { (\$bn) }\end{array}$ \\
\hline \multirow[t]{3}{*}{ China, PRC (CHN) } & 1992 & 0.0 & 52.3 & 0.4 & 1.4 & 0.5 & 0.2 & 1.4 & 0.6 & 0.6 & 0.1 & 9.1 & 3.2 & 67.0 & 57.9 & 12.2 & 11.1 & 9.5 & 11.3 & 65.5 \\
\hline & 1996 & 0.0 & 23.2 & 0.7 & 3.4 & 0.8 & 0.5 & 2.4 & 0.7 & 1.6 & 0.5 & 17.8 & 5.6 & 52.1 & 34.2 & 21.5 & 20.2 & 14.0 & 12.4 & 123.6 \\
\hline & 2000 & 0.0 & 18.6 & 1.0 & 3.4 & 0.9 & 0.5 & 2.2 & 0.8 & 1.7 & 0.5 & 15.1 & 6.0 & 45.0 & 29.9 & 24.8 & 22.9 & 16.2 & 14.1 & 215.5 \\
\hline \multirow[t]{3}{*}{ Hong Kong (HKG) } & 1992 & 45.5 & 0.0 & 0.5 & 1.9 & 2.0 & 0.0 & 5.1 & 1.1 & 0.0 & 0.0 & 4.2 & 8.7 & 60.5 & 56.2 & 27.8 & 24.2 & 6.0 & 5.8 & 40.3 \\
\hline & 1996 & 28.0 & 0.0 & 0.7 & 1.1 & 1.2 & 1.1 & 4.6 & 1.2 & 3.1 & 0.2 & 4.8 & 9.0 & 46.3 & 41.4 & 28.9 & 26.9 & 18.5 & 6.4 & 25.6 \\
\hline & 2000 & 29.4 & 0.0 & 0.4 & 0.8 & 1.5 & 1.2 & 2.7 & 0.7 & 3.4 & 0.1 & 2.7 & 6.6 & 43.2 & 40.5 & 33.5 & 31.2 & 18.9 & 4.4 & 22.1 \\
\hline \multirow[t]{3}{*}{ Indonesia (IND) } & 1992 & 4.0 & 3.8 & 0.0 & 3.3 & 1.4 & 0.6 & 13.5 & 0.9 & 2.9 & 1.2 & 12.7 & 17.7 & 44.4 & 31.8 & 19.2 & 17.9 & 21.9 & 14.4 & 15.8 \\
\hline & 1996 & 2.1 & 5.1 & 0.0 & 3.0 & 2.7 & 1.1 & 11.1 & 1.5 & 2.6 & 1.3 & 15.9 & 17.6 & 46.6 & 30.7 & 19.6 & 18.4 & 19.3 & 14.5 & 25.2 \\
\hline & 2000 & 3.0 & 3.5 & 0.0 & 2.2 & 3.8 & 1.4 & 13.2 & 1.8 & 2.5 & 0.8 & 13.2 & 20.9 & 45.6 & 32.4 & 20.6 & 19.2 & 18.5 & 15.3 & 34.7 \\
\hline \multirow[t]{3}{*}{ Korea, Rp of (KOR) } & 1992 & 2.5 & 7.6 & 2.5 & 0.0 & 1.5 & 0.9 & 4.2 & 1.6 & 2.5 & 0.5 & 11.7 & 11.2 & 35.6 & 23.9 & 29.5 & 25.9 & 14.3 & 20.5 & 66.3 \\
\hline & 1996 & 8.2 & 7.7 & 2.5 & 0.0 & 2.9 & 1.5 & 3.8 & 1.8 & 2.7 & 1.3 & 9.2 & 13.9 & 41.8 & 32.6 & 21.1 & 19.0 & 13.6 & 23.5 & 109.7 \\
\hline & 2000 & 9.7 & 5.6 & 1.9 & 0.0 & 2.1 & 1.9 & 3.3 & 1.2 & 4.8 & 1.0 & 9.0 & 11.4 & 40.6 & 31.5 & 26.8 & 23.9 & 15.3 & 17.3 & 148.3 \\
\hline \multirow[t]{3}{*}{ Malaysia (MAL) } & 1992 & 1.0 & 5.1 & 0.6 & 1.0 & 0.0 & 0.7 & 26.0 & 2.1 & 2.6 & 0.1 & 7.7 & 29.5 & 47.2 & 39.5 & 28.8 & 27.1 & 18.0 & 6.0 & 25.9 \\
\hline & 1996 & 1.7 & 6.7 & 1.4 & 1.5 & 0.0 & 1.1 & 22.4 & 2.9 & 4.3 & 0.4 & 11.1 & 28.0 & 53.8 & 42.7 & 24.2 & 23.0 & 15.1 & 6.9 & 58.7 \\
\hline & 2000 & 2.5 & 5.1 & 1.3 & 2.3 & 0.0 & 1.6 & 19.7 & 3.0 & 3.6 & 0.4 & 11.3 & 26.1 & 51.1 & 39.8 & 26.2 & 24.5 & 15.1 & 7.5 & 78.3 \\
\hline \multirow[t]{3}{*}{ Philippines (PHL) } & 1992 & 1.0 & 0.0 & 0.5 & 1.3 & 3.3 & 0.0 & 4.3 & 1.7 & 0.0 & 0.0 & 13.1 & 9.8 & 25.2 & 12.1 & 68.7 & 65.5 & 4.8 & 1.3 & 5.8 \\
\hline & 1996 & 0.4 & 4.2 & 0.4 & 1.1 & 3.8 & 0.0 & 6.5 & 4.3 & 3.3 & 0.6 & 16.1 & 15.6 & 40.7 & 24.7 & 38.2 & 37.1 & 17.4 & 3.6 & 16.9 \\
\hline & 2000 & 1.2 & 5.0 & 0.4 & 2.7 & 3.7 & 0.0 & 8.3 & 3.3 & 7.9 & 0.2 & 13.7 & 15.8 & 46.4 & 32.7 & 32.8 & 31.1 & 18.3 & 2.5 & 34.8 \\
\hline \multirow[t]{3}{*}{ Singapore (SGP) } & 1992 & 1.3 & 6.8 & 1.6 & 1.9 & 11.6 & 1.2 & 0.0 & 5.4 & 4.7 & 0.4 & 5.5 & 20.2 & 40.5 & 35.0 & 27.5 & 26.4 & 19.0 & 13.0 & 48.1 \\
\hline & 1996 & 1.9 & 7.3 & 0.0 & 2.9 & 18.3 & 1.9 & 0.0 & 5.7 & 4.0 & 0.9 & 7.7 & 26.8 & 52.7 & 45.0 & 22.5 & 21.7 & 14.8 & 10.0 & 103.4 \\
\hline & 2000 & 3.7 & 6.8 & 1.5 & 3.8 & 18.2 & 2.3 & 0.0 & 4.6 & 6.4 & 0.8 & 7.5 & 27.4 & 54.8 & 47.3 & 20.8 & 19.7 & 14.6 & 9.7 & 117.1 \\
\hline \multirow[t]{3}{*}{ Thailand (THA) } & 1992 & 0.3 & 5.1 & 0.4 & 0.8 & 2.5 & 0.6 & 10.7 & 0.0 & 1.9 & 0.2 & 14.0 & 14.4 & 37.5 & 23.4 & 28.0 & 26.4 & 21.3 & 13.3 & 21.5 \\
\hline & 1996 & 2.2 & 5.8 & 1.1 & 1.3 & 4.3 & 1.1 & 16.6 & 0.0 & 0.0 & 0.0 & 15.7 & 23.1 & 48.2 & 32.5 & 26.0 & 19.6 & 19.6 & 6.2 & 38.4 \\
\hline & 2000 & 3.1 & 5.1 & 1.6 & 1.7 & 4.2 & 1.8 & 8.6 & 0.0 & 4.0 & 1.1 & \begin{tabular}{|l|}
13.9 \\
\end{tabular} & 17.4 & 46.6 & 32.7 & 24.5 & 22.9 & 17.7 & 11.2 & 50.9 \\
\hline
\end{tabular}




\begin{tabular}{|c|c|c|c|c|c|c|c|c|c|c|c|c|c|c|c|c|c|c|c|c|}
\hline Taiwan (TWN) & 1992 & 10.2 & 0.0 & 2.0 & 2.1 & 3.9 & 0.0 & 4.9 & 3.4 & 0.0 & 0.0 & 10.5 & 14.2 & 37.0 & 26.5 & 50.9 & 47.1 & 7.3 & 4.9 & 52.9 \\
\hline & 1996 & 12.6 & 12.6 & 1.3 & 2.1 & 2.9 & 1.3 & 4.3 & 2.4 & 0.0 & 0.0 & 9.2 & 12.1 & 48.6 & 39.4 & 28.5 & 14.9 & 14.9 & 8.0 & 114.8 \\
\hline & 2000 & 14.4 & 9.7 & 0.7 & 2.9 & 2.7 & 1.3 & 3.7 & 1.7 & 0.0 & 0.0 & 10.2 & 10.9 & 47.3 & 37.1 & 29.8 & 26.4 & 16.3 & 6.5 & 150.7 \\
\hline \multirow[t]{3}{*}{ Vietnam (VNM) } & 1992 & 1.5 & 0.0 & 0.0 & 8.6 & 2.0 & 0.0 & 2.5 & 1.5 & 0.0 & 0.0 & 58.9 & 6.1 & 75.6 & 16.8 & 1.5 & 0.0 & 14.7 & 8.1 & 0.2 \\
\hline & 1996 & 0.3 & 2.0 & 0.4 & 4.5 & 1.1 & 1.1 & 3.7 & 0.8 & 0.0 & 0.0 & 25.4 & 7.0 & 39.3 & 13.9 & 4.2 & 50.6 & 50.6 & 6.0 & 3.2 \\
\hline & 2000 & 1.0 & 1.9 & 0.2 & 2.7 & 1.9 & 0.4 & 2.0 & 3.5 & 0.0 & 0.0 & 20.9 & 8.5 & 34.6 & 13.7 & 5.4 & 3.6 & 52.1 & 7.8 & 6.6 \\
\hline \multirow[t]{3}{*}{ Japan (JPN) } & 1992 & 3.1 & 5.9 & 1.5 & 4.9 & 2.3 & 1.0 & 3.7 & 2.8 & 5.8 & 0.1 & 0.0 & 11.3 & 31.1 & 31.1 & 32.7 & 29.3 & 20.8 & 15.4 & 312.8 \\
\hline & 1996 & 4.9 & 5.8 & 2.1 & 6.7 & 3.6 & 2.1 & 4.9 & 4.2 & 5.9 & 0.3 & 0.0 & 17.3 & 40.7 & 40.7 & 30.8 & 28.6 & 16.2 & 12.4 & 374.7 \\
\hline & 2000 & 5.9 & 5.5 & 1.5 & 5.8 & 2.8 & 2.1 & 4.2 & 2.6 & 7.3 & 0.3 & 0.0 & 13.6 & 38.2 & 38.2 & 33.9 & 31.2 & 17.1 & 10.9 & 435.1 \\
\hline
\end{tabular}

\section{(B ) Manufacturing Exports net of pasts and components}

\begin{tabular}{|c|c|c|c|c|c|c|c|c|c|c|c|c|c|c|c|c|c|c|c|c|}
\hline & & $\mathrm{CHN}$ & $\mathrm{HKG}$ & IDN & KOR & MAL & PHL & SGP & THA & TWN & VNM & JPN & ASEAN & EAS & EAS-J & NAF & USA & $\mathrm{EU}$ & Other & WLD \\
\hline \multirow[t]{3}{*}{ China, PRC (CHN) } & 1992 & 0.0 & 51.6 & 0.4 & 1.4 & 0.5 & 0.2 & 1.3 & 0.7 & 0.5 & 0.1 & 9.2 & 3.1 & 66.1 & 57.0 & 12.5 & 11.4 & 9.9 & 11.4 & 61.1 \\
\hline & 1996 & 0.0 & 22.9 & 0.7 & 3.2 & 0.7 & 0.4 & 2.1 & 0.7 & 1.3 & 0.5 & 17.4 & 5.1 & 50.3 & 32.8 & 22.2 & 20.8 & 14.6 & 12.9 & 111.5 \\
\hline & 2000 & 0.0 & 17.1 & 1.0 & 3.1 & 0.8 & 0.4 & 1.6 & 0.6 & 1.5 & 0.6 & 14.9 & 5.0 & 41.8 & 26.9 & 26.3 & 24.3 & 16.9 & 15.1 & 184.2 \\
\hline \multirow[t]{3}{*}{ Hong Kong (HKG) } & 1992 & 43.7 & 0.0 & 0.5 & 0.5 & 1.7 & 0.0 & 3.5 & 1.1 & 0.0 & 0.0 & 4.2 & 6.8 & 55.2 & 51.1 & 31.2 & 27.0 & 7.0 & 6.6 & 31.6 \\
\hline & 1996 & 24.4 & 0.0 & 0.7 & 0.9 & 1.1 & 0.9 & 1.7 & 1.1 & 2.8 & 0.3 & 4.9 & 5.8 & 39.0 & 34.1 & 33.0 & 30.7 & 21.1 & 7.0 & 18.8 \\
\hline & 2000 & 25.9 & 0.0 & 0.5 & 0.6 & 0.9 & 1.0 & 1.1 & 0.7 & 2.6 & 0.1 & 2.0 & 4.2 & 35.8 & 33.8 & 39.0 & 36.3 & 20.5 & 4.7 & 16.4 \\
\hline \multirow[t]{3}{*}{ Indonesia (IND) } & 1992 & 4.2 & 3.8 & 0.0 & 3.4 & 1.4 & 0.7 & 12.9 & 0.8 & 2.9 & 1.2 & 12.5 & 17.0 & 43.8 & 31.3 & 19.4 & 18.0 & 22.2 & 14.6 & 15.2 \\
\hline & 1996 & 2.3 & 5.1 & 0.0 & 3.1 & 2.3 & 1.1 & 9.2 & 1.4 & 2.6 & 1.4 & 16.0 & 15.3 & 44.7 & 28.7 & 20.2 & 19.0 & 20.1 & 15.0 & 23.3 \\
\hline & 2000 & 3.3 & 3.6 & 0.0 & 2.3 & 2.9 & 1.3 & 10.0 & 1.7 & 2.6 & 0.9 & 12.2 & 16.7 & 40.9 & 28.7 & 21.9 & 20.3 & 20.1 & 17.1 & 29.8 \\
\hline \multirow[t]{3}{*}{ Korea, Rp of (KOR) } & 1992 & 2.7 & 7.4 & 2.8 & 0.0 & 0.8 & 0.9 & 1.7 & 1.6 & 2.2 & 0.6 & 11.7 & 8.4 & 32.4 & 20.8 & 28.9 & 25.1 & 15.0 & 23.6 & 54.5 \\
\hline & 1996 & 9.5 & 7.7 & 2.8 & 0.0 & 1.2 & 1.5 & 2.1 & 1.9 & 2.4 & 1.5 & 8.3 & 11.1 & 39.2 & 30.9 & 17.9 & 15.9 & 13.9 & 29.1 & 82.1 \\
\hline & 2000 & 10.2 & 5.2 & 2.2 & 0.0 & 1.0 & 1.2 & 1.6 & 1.0 & 2.9 & 1.2 & 7.9 & 8.2 & 34.5 & 26.7 & 27.0 & 23.9 & 16.2 & 22.2 & 102.9 \\
\hline \multirow[t]{3}{*}{ Malaysia (MAL) } & 1992 & 1.5 & 4.3 & 0.8 & 0.5 & 0.0 & 0.8 & 23.9 & 1.5 & 2.0 & 0.2 & 7.0 & 27.1 & 42.9 & 35.9 & 27.8 & 25.6 & 20.4 & 8.8 & 15.4 \\
\hline & 1996 & 2.5 & 6.7 & 1.7 & 1.7 & 0.0 & 1.1 & 15.7 & 1.9 & 2.6 & 0.5 & 12.6 & 20.9 & 47.8 & 35.2 & 25.3 & 23.7 & 16.9 & 10.0 & 33.8 \\
\hline & 2000 & 2.4 & 4.8 & 1.8 & 1.8 & 0.0 & 1.1 & 12.5 & 2.0 & 2.8 & 0.6 & 13.4 & 18.1 & 43.7 & 30.3 & 27.6 & 25.6 & 16.9 & 11.8 & 39.4 \\
\hline \multirow[t]{3}{*}{ Philippines (PHL) } & 1992 & 1.3 & 0.0 & 0.6 & 0.9 & 0.4 & 0.0 & 2.0 & 1.8 & 0.0 & 0.0 & 13.6 & 4.9 & 20.7 & 7.1 & 72.0 & 68.6 & 5.8 & 1.5 & 4.1 \\
\hline & 1996 & 0.7 & 3.9 & 0.7 & 0.7 & 0.6 & 0.0 & 2.4 & 0.7 & 2.4 & 1.3 & 17.3 & 5.7 & 30.7 & 13.5 & 46.1 & 44.3 & 16.2 & 7.0 & 8.0 \\
\hline & 2000 & 0.6 & 2.9 & 0.5 & 1.7 & 0.6 & 0.0 & 3.2 & 0.7 & 6.1 & 0.4 & 18.0 & 5.5 & 34.9 & 16.9 & 43.3 & 41.5 & 17.0 & 4.8 & 12.5 \\
\hline
\end{tabular}




\begin{tabular}{|c|c|c|c|c|c|c|c|c|c|c|c|c|c|c|c|c|c|c|c|c|}
\hline \multirow[t]{3}{*}{ Singapore (SGP) } & 1992 & 1.3 & 6.5 & 1.8 & 1.8 & 9.8 & 1.3 & 0.0 & 4.2 & 4.0 & 0.5 & 4.8 & 17.6 & 36.4 & 31.6 & 27.8 & 26.5 & 21.3 & 14.5 & 34.5 \\
\hline & 1996 & 1.8 & 7.5 & 0.0 & 3.0 & 14.3 & 1.8 & 0.0 & 5.5 & 3.4 & 1.3 & 8.0 & 22.8 & 49.6 & 41.6 & 23.0 & 22.2 & 15.1 & 12.4 & 62.3 \\
\hline & 2000 & 3.7 & 6.0 & 2.5 & 3.8 & 13.3 & 2.4 & 0.0 & 4.4 & 5.3 & 1.1 & 8.8 & 23.7 & 49.9 & 41.1 & 21.9 & 21.0 & 15.2 & 13.0 & 59.0 \\
\hline \multirow[t]{3}{*}{ Thailand (THA) } & 1992 & 0.3 & 5.5 & 0.5 & 0.7 & 1.4 & 0.5 & 5.2 & 0.0 & 1.9 & 0.2 & 13.2 & 7.8 & 30.5 & 17.3 & 29.6 & 27.7 & 23.9 & 16.0 & 16.9 \\
\hline & 1996 & 2.1 & 6.3 & 1.2 & 1.2 & 2.3 & 1.0 & 13.0 & 0.0 & 0.0 & 0.0 & \begin{tabular}{l|l|}
0 & 16.4 \\
\end{tabular} & 17.4 & 43.3 & 26.9 & 28.4 & 21.4 & 21.4 & 7.0 & 29.4 \\
\hline & 2000 & 2.2 & 5.3 & 1.9 & 1.0 & 2.8 & 1.4 & 4.2 & 0.0 & 2.4 & 1.4 & 13.5 & 11.7 & 38.2 & 24.7 & 28.0 & 26.3 & 18.7 & 15.1 & 32.6 \\
\hline \multirow[t]{3}{*}{ Taiwan (TWN) } & 1992 & 10.4 & 0.0 & 2.1 & 1.7 & 3.6 & 0.0 & 4.1 & 3.2 & 0.0 & 0.0 & 10.6 & 13.0 & 35.8 & 25.2 & 51.9 & 48.0 & 7.2 & 5.1 & 42.3 \\
\hline & 1996 & 14.7 & 12.3 & 1.5 & 1.6 & 2.6 & 1.4 & 3.3 & 2.4 & 0.0 & 0.0 & 9.3 & 11.2 & 49.1 & 39.8 & 26.9 & 15.2 & 15.2 & 8.8 & 81.7 \\
\hline & 2000 & 16.3 & 8.1 & 1.0 & 1.6 & 1.8 & 1.0 & 2.0 & 1.9 & 0.0 & 0.0 & 9.6 & 9.0 & 43.4 & 33.8 & 32.1 & 28.6 & 17.0 & 7.5 & 93.7 \\
\hline \multirow[t]{3}{*}{ Vietnam (VNM) } & 1992 & 1.0 & 0.0 & 0.0 & 8.3 & 2.1 & 0.0 & 2.6 & 1.6 & 0.0 & 0.0 & 59.6 & 6.2 & 75.1 & 15.5 & 1.6 & 0.0 & 15.0 & 8.3 & 0.2 \\
\hline & 1996 & 0.3 & 1.8 & 0.1 & 4.3 & 0.5 & 0.2 & 2.8 & 0.5 & 0.0 & 0.0 & \begin{tabular}{|l|l|}
0 & 26.0 \\
\end{tabular} & 4.1 & 36.4 & 10.5 & 4.4 & 53.1 & 53.1 & 6.1 & 3.0 \\
\hline & 2000 & 1.0 & 1.8 & 0.1 & 2.6 & 0.8 & 0.4 & 1.7 & 0.4 & 0.0 & 0.0 & \begin{tabular}{l|l|}
0 & 20.4 \\
\end{tabular} & 4.0 & 29.4 & 8.9 & 5.9 & 3.9 & 56.5 & 8.2 & 6.1 \\
\hline \multirow[t]{3}{*}{ Japan (JPN) } & 1992 & 3.5 & 6.2 & 1.5 & 4.3 & 1.7 & 0.9 & 3.0 & 2.5 & 5.0 & 0.2 & 0.0 & 9.8 & 29.0 & 29.0 & 31.5 & 28.2 & 21.8 & 17.7 & 241.1 \\
\hline & 1996 & 5.4 & 5.9 & 2.1 & 6.6 & 2.6 & 1.6 & 4.0 & 4.0 & 5.2 & 0.4 & 0.0 & 14.7 & 38.0 & 38.0 & 29.8 & 27.9 & 17.0 & 15.2 & 261.7 \\
\hline & 2000 & 5.7 & 4.9 & 1.2 & 5.5 & 1.9 & 1.4 & 3.3 & 2.2 & 7.3 & 0.4 & 0.0 & 10.4 & 33.9 & 33.9 & 35.0 & 32.5 & 17.8 & 13.3 & 302.1 \\
\hline \multicolumn{21}{|c|}{ (C) Manufacturing Imports } \\
\hline & & $\mathrm{CHN}$ & HKG & IDN & KOR & MAL & PHL & SGP & THA & TWN & VNM & JPN & ASEAN & EAS & EAS-J & NAF & USA & $\mathrm{EU}$ & Other & WLD \\
\hline \multirow[t]{3}{*}{ China, PRC (CHN) } & 1992 & 0.0 & 30.4 & 1.1 & 2.8 & 0.4 & 0.1 & 0.6 & 0.2 & 8.9 & 0.0 & \begin{tabular}{l|l|}
0 & 18.5 \\
\end{tabular} & 2.3 & 63.1 & 44.6 & 12.6 & 11.3 & 15.7 & 8.7 & 60.3 \\
\hline & 1996 & 0.0 & 7.1 & 0.7 & 10.1 & 1.3 & 0.1 & 1.9 & 0.8 & 14.2 & 0.0 & 24.5 & 4.8 & 60.8 & 36.2 & 13.3 & 12.2 & 17.5 & 8.4 & 102.4 \\
\hline & 2000 & 0.0 & 5.4 & 1.2 & 11.2 & 2.5 & 0.8 & 2.5 & 1.8 & 13.5 & 0.0 & \begin{tabular}{l|l|}
0 & 22.7 \\
\end{tabular} & 8.9 & 61.8 & 39.1 & 12.5 & 11.0 & 17.0 & 8.7 & 160.2 \\
\hline \multirow[t]{3}{*}{ Hong Kong (HKG) } & 1992 & 46.1 & 0.0 & 0.8 & 6.8 & 1.8 & 0.0 & 4.4 & 1.5 & 0.0 & 0.0 & \begin{tabular}{l|l|}
0 & 24.9 \\
\end{tabular} & 8.4 & 86.2 & 61.3 & 8.1 & 7.7 & 1.5 & 4.2 & 74.4 \\
\hline & 1996 & 40.2 & 0.0 & 0.7 & 4.9 & 2.2 & 0.4 & 4.2 & 1.3 & 8.4 & 0.0 & \begin{tabular}{l|l|}
0 & 14.1 \\
\end{tabular} & 8.9 & 76.5 & 62.4 & 7.8 & 7.3 & 10.9 & 4.8 & 170.8 \\
\hline & 2000 & 46.0 & 0.0 & 0.6 & 4.4 & 2.3 & 1.0 & 4.0 & 1.4 & 7.7 & 0.1 & \begin{tabular}{l|l|} 
& 12.5 \\
\end{tabular} & 9.5 & 80.0 & 67.5 & 7.1 & 6.5 & 8.5 & 4.5 & 189.5 \\
\hline \multirow[t]{3}{*}{ Indonesia (IND) } & 1992 & 2.2 & 1.0 & 0.0 & 8.4 & 1.0 & 0.2 & 4.1 & 0.6 & 5.5 & 0.0 & 26.6 & 5.9 & 49.7 & 23.1 & 16.6 & 15.4 & 27.3 & 6.4 & 19.3 \\
\hline & 1996 & 3.0 & 0.7 & 0.0 & 7.0 & 1.8 & 0.2 & 4.7 & 1.5 & 5.1 & 0.0 & \begin{tabular}{l|l|}
0 & 26.3 \\
\end{tabular} & 8.3 & 50.5 & 24.2 & 13.9 & 12.5 & 29.1 & 6.5 & 28.3 \\
\hline & 2000 & 5.2 & 1.5 & 0.0 & 7.7 & 3.0 & 0.4 & 9.0 & 3.2 & 5.8 & 0.1 & 24.3 & 15.7 & 60.3 & 36.0 & 13.2 & 12.1 & 17.7 & 8.8 & 18.9 \\
\hline \multirow[t]{3}{*}{ Korea, $\mathrm{Rp}$ of (KOR) } & 1992 & 3.5 & 1.6 & 1.3 & 0.0 & 0.7 & 0.2 & 2.9 & 0.4 & 2.2 & 0.0 & \begin{tabular}{l|l|}
0 & 34.9 \\
\end{tabular} & 5.5 & 47.7 & 12.8 & 27.4 & 26.3 & 18.8 & 6.0 & 48.4 \\
\hline & 1996 & 5.3 & 1.1 & 0.9 & 0.0 & 1.4 & 0.3 & 2.2 & 0.6 & 2.7 & 0.2 & \begin{tabular}{l|l|}
2 & 30.2 \\
\end{tabular} & 5.6 & 44.9 & 14.7 & 28.5 & 27.1 & 19.9 & 6.7 & 90.4 \\
\hline & 2000 & 8.9 & 1.2 & 0.9 & 0.0 & 3.4 & 1.6 & 3.3 & 1.2 & 4.6 & 0.2 & \begin{tabular}{l|l}
2 & 29.1
\end{tabular} & 10.7 & 54.5 & 25.5 & 26.7 & 25.5 & 14.2 & 4.6 & 93.1 \\
\hline
\end{tabular}




\begin{tabular}{|c|c|c|c|c|c|c|c|c|c|c|c|c|c|c|c|c|c|c|c|c|}
\hline \multirow[t]{3}{*}{ Malaysia (MAL) } & 1992 & 1.5 & 2.5 & 0.9 & 3.2 & 0.0 & 0.6 & 14.7 & 1.9 & 6.6 & 0.0 & 29.5 & 18.2 & 61.6 & 32.2 & 19.4 & 18.8 & 15.0 & 4.0 & 31.2 \\
\hline & 1996 & 2.2 & 2.7 & 1.4 & 5.2 & 0.0 & 1.2 & 13.0 & 2.7 & 5.4 & 0.1 & 27.6 & 18.3 & 61.5 & 33.9 & 18.3 & 17.6 & 16.1 & 4.1 & 61.1 \\
\hline & 2000 & 3.7 & 3.2 & 2.3 & 4.8 & 0.0 & 2.9 & 13.7 & 3.5 & 6.2 & 0.2 & 23.1 & 22.7 & 63.7 & 40.6 & 20.0 & 19.5 & 12.0 & 4.3 & 66.0 \\
\hline \multirow[t]{3}{*}{ Philippines (PHL) } & 1992 & 1.8 & 0.0 & 1.4 & 8.2 & 2.4 & 0.0 & 8.2 & 1.9 & 0.0 & 0.0 & 42.9 & 13.9 & 66.8 & 23.9 & 27.2 & 26.4 & 3.8 & 2.2 & 7.2 \\
\hline & 1996 & 1.9 & 5.5 & 1.2 & 6.3 & 2.3 & 0.0 & 5.8 & 1.6 & 5.8 & 0.1 & 28.2 & 11.0 & 58.7 & 30.5 & 23.2 & 22.5 & 13.6 & 4.4 & 25.3 \\
\hline & 2000 & 2.4 & 5.0 & 1.9 & 8.7 & 3.6 & 0.0 & 7.5 & 3.3 & 7.8 & 0.1 & 24.3 & 16.4 & 64.6 & 40.3 & 19.7 & 19.2 & 11.2 & 4.6 & 24.7 \\
\hline \multirow[t]{3}{*}{ Singapore (SGP) } & 1992 & 2.1 & 3.9 & 4.0 & 3.8 & 14.2 & 0.5 & 0.0 & 4.2 & 4.9 & 0.0 & 25.8 & 22.9 & 59.3 & 33.5 & 20.5 & 20.0 & 15.1 & 5.1 & 53.2 \\
\hline & 1996 & 3.4 & 3.7 & 0.0 & 3.8 & 15.7 & 1.2 & 0.0 & 6.0 & 4.6 & 0.1 & 20.8 & 23.1 & 59.5 & 38.7 & 19.3 & 18.6 & 15.6 & 5.5 & 105.9 \\
\hline & 2000 & 5.5 & 3.1 & 4.2 & 3.9 & 18.8 & 3.0 & 0.0 & 4.4 & 5.2 & 0.1 & 20.0 & 30.5 & 64.2 & 44.2 & 18.1 & 17.4 & 12.1 & 5.5 & 108.1 \\
\hline \multirow[t]{3}{*}{ Thailand (THA) } & 1992 & 2.2 & 1.7 & 0.4 & 4.2 & 2.2 & 0.4 & 6.4 & 0.0 & 6.6 & 0.0 & 36.2 & 9.4 & 60.2 & 24.0 & 14.5 & 13.5 & 19.0 & 6.3 & 27.5 \\
\hline & 1996 & 2.8 & 1.6 & 1.0 & 3.8 & 3.9 & 0.9 & 5.7 & 0.0 & 5.3 & 0.1 & 34.9 & 11.5 & 59.9 & 25.0 & 15.3 & 14.6 & 17.8 & 6.9 & 51.9 \\
\hline & 2000 & 6.4 & 1.8 & 1.6 & 4.3 & 5.9 & 2.4 & 7.1 & 0.0 & 5.9 & 0.5 & 30.4 & 17.5 & 66.3 & 35.9 & 14.9 & 14.3 & 12.2 & 6.6 & 43.8 \\
\hline \multirow[t]{3}{*}{ Taiwan (TWN) } & 1992 & 1.1 & 0.0 & 1.2 & 4.5 & 1.8 & 0.0 & 6.1 & 1.1 & 0.0 & 0.0 & 47.9 & 10.2 & 63.6 & 15.7 & 28.5 & 27.7 & 2.9 & 4.9 & 37.6 \\
\hline & 1996 & 3.2 & 1.3 & 1.1 & 4.9 & 4.2 & 0.9 & 6.9 & 0.0 & 0.0 & 0.0 & 36.9 & 13.0 & 59.4 & 22.5 & 21.0 & 15.9 & 15.9 & 3.7 & 60.4 \\
\hline & 2000 & 4.0 & 0.8 & 0.9 & 7.5 & 3.0 & 2.9 & 8.0 & 2.1 & 0.0 & 0.0 & 33.8 & 17.9 & 63.2 & 29.4 & 20.8 & 20.3 & 11.9 & 4.1 & 93.6 \\
\hline \multirow[t]{3}{*}{ Vietnam (VNM) } & 1992 & 4.2 & 0.0 & 14.1 & 26.8 & 2.4 & 0.0 & 14.8 & 2.8 & 0.0 & 0.0 & 30.1 & 34.1 & 95.2 & 65.1 & 0.6 & 0.1 & 1.5 & 2.6 & 1.3 \\
\hline & 1996 & 8.5 & 0.9 & 4.6 & 20.0 & 3.0 & 1.5 & 13.5 & 0.0 & 0.0 & 0.0 & 14.1 & 22.6 & 66.0 & 51.9 & 8.1 & 20.4 & 20.4 & 5.5 & 7.1 \\
\hline & 2000 & 14.1 & 0.4 & 3.5 & 19.1 & 4.3 & 0.7 & 11.8 & 7.5 & 0.0 & 0.0 & 19.1 & 28.3 & 80.5 & 61.4 & 3.6 & 3.3 & 12.0 & 3.9 & 7.8 \\
\hline \multirow[t]{3}{*}{ Japan (JPN) } & 1992 & 10.2 & 1.7 & 2.1 & 7.6 & 1.8 & 0.8 & 1.8 & 3.0 & 5.6 & 0.1 & 0.0 & 9.6 & 34.7 & 34.7 & 30.9 & 29.7 & 27.3 & 7.1 & 99.2 \\
\hline & 1996 & 16.3 & 1.1 & 2.3 & 5.6 & 3.5 & 1.7 & 3.0 & 3.3 & 5.7 & 0.4 & 0.0 & 14.2 & 42.9 & 42.9 & 29.0 & 27.7 & 22.5 & 5.6 & 185.4 \\
\hline & 2000 & 20.9 & 0.7 & 2.3 & 6.3 & 4.6 & 2.7 & 2.5 & 3.4 & 7.4 & 0.7 & 0.0 & 16.1 & 51.4 & 51.4 & 25.7 & 24.1 & 18.5 & 4.5 & 209.2 \\
\hline
\end{tabular}

(D) Manufacturing imports net of parts and components

\begin{tabular}{|c|c|c|c|c|c|c|c|c|c|c|c|c|c|c|c|c|c|c|c|c|}
\hline & & CHN & HKG & IDN & KOR & MAL & PHL & SGP & THA & TWN & VNM & JPN & ASEAN & EAS & EAS-J & $\mathrm{NAF}$ & USA & $\mathrm{EU}$ & Other & WLD \\
\hline \multirow[t]{3}{*}{ China, PRC (CHN) } & 1992 & 0.0 & 28.5 & 1.4 & 3.1 & 0.4 & 0.1 & 0.6 & 0.2 & 9.0 & 0.0 & 18.3 & 2.7 & 61.6 & 43.4 & 13.2 & 11.8 & 15.4 & 9.8 & 48.5 \\
\hline & 1996 & 0.0 & 6.5 & 0.9 & 10.8 & 1.2 & 0.1 & 1.4 & 0.8 & 14.9 & 0.0 & 21.9 & 4.3 & 58.5 & 36.6 & 14.0 & 12.8 & 18.3 & 9.2 & 80.8 \\
\hline & 2000 & 0.0 & 5.2 & 1.5 & 12.2 & 1.5 & 0.3 & 2.2 & 1.5 & 14.3 & 0.1 & 21.4 & 7.1 & 60.3 & 38.9 & 13.5 & 12.3 & 16.8 & 9.5 & 106.6 \\
\hline \multirow[t]{3}{*}{ Hong Kong (HKG) } & 1992 & 49.9 & 0.0 & 0.9 & 6.4 & 1.0 & 0.0 & 3.6 & 1.5 & 0.0 & 0.0 & 23.5 & 7.0 & 86.8 & 63.3 & 7.1 & 6.7 & 1.4 & 4.7 & 63.2 \\
\hline & 1996 & 45.4 & 0.0 & 0.8 & 4.6 & 1.8 & 0.2 & 3.2 & 1.4 & 7.4 & 0.0 & 11.7 & 7.4 & 76.4 & 64.8 & 6.6 & 6.1 & 11.4 & 5.6 & 136.0 \\
\hline & 2000 & 54.0 & 0.0 & 0.8 & 4.0 & 1.5 & 0.4 & 2.3 & 1.3 & 5.6 & 0.1 & 9.7 & 6.3 & 79.6 & 69.9 & 5.6 & 5.2 & 9.0 & 5.8 & 136.1 \\
\hline
\end{tabular}




\begin{tabular}{|c|c|c|c|c|c|c|c|c|c|c|c|c|c|c|c|c|c|c|c|c|}
\hline Indonesia (IND) & 1992 & 2.6 & 1.0 & 0.0 & 9.4 & 1.0 & 0.2 & 4.0 & 0.6 & 5.7 & 0.0 & 24.5 & 5.8 & 49.0 & 24.5 & 16.2 & 15.0 & 27.7 & 7.0 & 15.3 \\
\hline & 1996 & 3.4 & 0.8 & 0.0 & 8.1 & 1.8 & 0.3 & 5.0 & 1.6 & 5.6 & 0.0 & 22.0 & 8.7 & 48.7 & 26.7 & 14.2 & 12.7 & 29.7 & 7.4 & 21.6 \\
\hline & 2000 & 5.8 & 1.7 & 0.0 & 8.8 & 3.2 & 0.4 & 9.7 & 3.5 & 6.3 & 0.0 & 19.5 & 16.8 & 58.8 & 39.3 & 13.0 & 12.1 & 18.6 & 9.6 & 15.2 \\
\hline \multirow[t]{3}{*}{ Korea, $\mathrm{Rp}$ of (KOR) } & 1992 & 4.5 & 0.5 & 1.7 & 0.0 & 0.5 & 0.1 & 1.2 & 0.4 & 2.1 & 0.0 & 32.3 & 3.9 & 43.3 & 11.0 & 27.2 & 25.9 & 21.8 & 7.7 & 35.5 \\
\hline & 1996 & 5.9 & 0.6 & 1.1 & 0.0 & 1.1 & 0.1 & 1.8 & 0.5 & 2.0 & 0.2 & 28.6 & 4.9 & 42.0 & 13.4 & 27.6 & 25.9 & 22.2 & 8.2 & 65.6 \\
\hline & 2000 & 10.6 & 0.6 & 1.4 & 0.0 & 1.6 & 0.6 & 3.2 & 1.0 & 2.7 & 0.3 & 29.8 & 8.0 & 51.8 & 22.0 & 24.7 & 23.2 & 17.2 & 6.3 & 56.9 \\
\hline \multirow[t]{3}{*}{ Malaysia (MAL) } & 1992 & 2.1 & 2.8 & 1.4 & 3.1 & 0.0 & 0.1 & 11.4 & 1.3 & 7.9 & 0.0 & 29.6 & 14.2 & 59.7 & 30.1 & 17.7 & 16.9 & 17.4 & 5.1 & 19.4 \\
\hline & 1996 & 3.2 & 2.8 & 1.9 & 3.1 & 0.0 & 0.2 & 9.3 & 2.1 & 6.6 & 0.0 & 28.6 & 13.5 & 57.9 & 29.2 & 16.3 & 15.1 & 20.3 & 5.6 & 32.1 \\
\hline & 2000 & 5.2 & 3.3 & 3.2 & 4.3 & 0.0 & 0.5 & 12.3 & 3.3 & 6.3 & 0.2 & 26.0 & 19.4 & 64.5 & 38.5 & 16.5 & 15.5 & 13.8 & 5.2 & 27.2 \\
\hline \multirow[t]{3}{*}{ Philippines (PHL) } & 1992 & 2.5 & 0.0 & 2.0 & 10.0 & 2.4 & 0.0 & 9.1 & 1.7 & 0.0 & 0.0 & 43.2 & 15.2 & 70.9 & 27.6 & 21.6 & 20.6 & 4.8 & 2.8 & 4.9 \\
\hline & 1996 & 2.8 & 6.1 & 2.0 & 7.8 & 2.0 & 0.0 & 5.5 & 2.0 & 7.9 & 0.0 & 23.9 & 11.6 & 60.2 & 36.3 & 18.8 & 17.7 & 15.0 & 6.1 & 14.3 \\
\hline & 2000 & 4.4 & 7.3 & 3.9 & 10.0 & 3.5 & 0.0 & 7.9 & 4.0 & 8.6 & 0.2 & 21.8 & 19.5 & 71.6 & 49.7 & 11.8 & 11.3 & 11.1 & 5.5 & 11.1 \\
\hline \multirow[t]{3}{*}{ Singapore (SGP) } & 1992 & 2.7 & 3.0 & 5.4 & 3.2 & 13.2 & 0.2 & 0.0 & 2.5 & 4.8 & 0.0 & 25.0 & 21.4 & 54.9 & 29.9 & 21.0 & 20.4 & 17.5 & 6.5 & 36.2 \\
\hline & 1996 & 4.3 & 3.4 & 0.0 & 2.9 & 13.3 & 0.4 & 0.0 & 6.3 & 4.5 & 0.1 & 20.4 & 20.1 & 55.7 & 35.3 & 18.1 & 17.4 & 18.5 & 7.7 & 60.6 \\
\hline & 2000 & 6.5 & 3.5 & 5.7 & 3.3 & 16.3 & 1.1 & 0.0 & 3.7 & 3.6 & 0.2 & 21.8 & 27.0 & 60.4 & 38.6 & 18.2 & 17.6 & 14.9 & 6.5 & 52.2 \\
\hline \multirow[t]{3}{*}{ Thailand (THA) } & 1992 & 2.8 & 1.7 & 0.6 & 4.6 & 1.2 & 0.4 & 4.1 & 0.0 & 6.8 & 0.0 & 35.2 & 6.2 & 57.2 & 22.1 & 13.8 & 12.7 & 21.3 & 7.7 & 20.2 \\
\hline & 1996 & 3.3 & 1.6 & 1.3 & 4.3 & 2.3 & 0.2 & 4.7 & 0.0 & 5.7 & 0.0 & 33.1 & 8.6 & 56.7 & 23.6 & 14.9 & 14.0 & 20.9 & 7.4 & 34.8 \\
\hline & 2000 & 7.1 & 2.0 & 2.0 & 4.7 & 4.1 & 0.7 & 6.8 & 0.0 & 6.8 & 0.1 & 30.3 & 13.8 & 64.6 & 34.3 & 11.6 & 11.0 & 15.4 & 8.4 & 26.4 \\
\hline \multirow[t]{3}{*}{ Taiwan (TWN) } & 1992 & 1.2 & 0.0 & 1.7 & 4.5 & 1.2 & 0.0 & 5.2 & 1.2 & 0.0 & 0.0 & 45.1 & 9.2 & 60.0 & 14.9 & 30.3 & 29.3 & 3.2 & 6.5 & 26.5 \\
\hline & 1996 & 3.7 & 1.4 & 1.6 & 5.0 & 2.3 & 0.5 & 5.4 & 0.0 & 0.0 & 0.0 & 34.8 & 9.7 & 54.5 & 19.7 & 21.6 & 18.9 & 18.9 & 5.0 & 39.2 \\
\hline & 2000 & 4.6 & 0.7 & 1.3 & 5.0 & 1.9 & 1.3 & 5.4 & 1.3 & 0.0 & 0.0 & 37.8 & 12.5 & 59.3 & 21.5 & 21.3 & 20.7 & 13.5 & 5.9 & 58.7 \\
\hline \multirow[t]{3}{*}{ Vietnam (VNM) } & 1992 & 4.2 & 0.0 & 14.7 & 27.0 & 2.3 & 0.0 & 14.4 & 2.8 & 0.0 & 0.0 & 30.6 & 34.2 & 96.0 & 65.4 & 0.6 & 0.1 & 1.0 & 2.4 & 1.3 \\
\hline & 1996 & 8.8 & 0.9 & 5.1 & 20.0 & 2.7 & 1.6 & 12.6 & 0.0 & 0.0 & 0.0 & 14.7 & 22.0 & 66.4 & 51.7 & 8.2 & 20.0 & 20.0 & 5.4 & 6.3 \\
\hline & 2000 & 16.2 & 0.4 & 4.1 & 20.4 & 3.9 & 0.7 & 10.5 & 7.1 & 0.0 & 0.0 & 17.8 & 26.9 & 81.2 & 63.4 & 2.8 & 2.4 & 12.0 & 4.0 & 6.3 \\
\hline \multirow[t]{3}{*}{ Japan (JPN) } & 1992 & 11.5 & 1.6 & 2.4 & 7.4 & 1.6 & 0.7 & 1.4 & 2.7 & 5.3 & 0.1 & 0.0 & 8.9 & 34.7 & 34.7 & 28.0 & 26.7 & 29.5 & 7.8 & 83.9 \\
\hline & 1996 & 18.2 & 1.0 & 2.6 & 4.4 & 3.4 & 1.3 & 2.5 & 3.2 & 5.1 & 0.5 & 0.0 & 13.5 & 42.2 & 42.2 & 26.1 & 24.7 & 25.2 & 6.5 & 149.6 \\
\hline & 2000 & 23.8 & 0.5 & 2.5 & 5.4 & 4.0 & 1.7 & 1.9 & 3.3 & 5.7 & 0.8 & 0.0 & 14.3 & 49.6 & 49.6 & 23.5 & 21.9 & 21.5 & 5.4 & 158.5 \\
\hline
\end{tabular}




\begin{tabular}{|c|c|c|c|c|c|c|c|c|c|c|c|c|c|c|c|c|c|c|c|c|}
\hline \multicolumn{21}{|c|}{ (E) Total trade (exports + imports) } \\
\hline & & CHN & $\mathrm{HKG}$ & IDN & KOR & MAL & PHL & SGP & THA & TWN & VNM & JPN & ASEAN & EAS & EAS-J & NAF & USA & $\mathrm{EU}$ & Other & WLD \\
\hline \multirow[t]{3}{*}{ China, PRC (CHN) } & 1992 & 0.0 & 41.8 & 0.7 & 2.1 & 0.4 & 0.2 & 1.0 & 0.4 & 4.6 & 0.0 & 13.6 & 2.8 & 65.1 & 51.5 & 12.4 & 11.2 & 12.5 & 10.0 & 125.7 \\
\hline & 1996 & 0.0 & 15.9 & 0.7 & 6.5 & 1.0 & 0.3 & 2.2 & 0.8 & 7.3 & 0.3 & 20.9 & 5.2 & 56.0 & 35.2 & 17.8 & 16.5 & 15.6 & 10.6 & 226.0 \\
\hline & 2000 & 0.0 & 13.0 & 1.1 & 6.7 & 1.6 & 0.7 & 2.3 & 1.2 & 6.8 & 0.3 & 18.3 & 7.2 & 52.1 & 33.8 & 19.5 & 17.8 & 16.5 & 11.8 & 375.7 \\
\hline \multirow[t]{3}{*}{ Hong Kong (HKG) } & 1992 & 45.9 & 0.0 & 0.7 & 5.1 & 1.8 & 0.0 & 4.7 & 1.4 & 0.0 & 0.0 & 17.7 & 8.5 & 77.2 & 59.5 & 15.0 & 13.5 & 3.1 & 4.8 & 114.7 \\
\hline & 1996 & 38.6 & 0.0 & 0.7 & 4.4 & 2.1 & 0.5 & 4.3 & 1.3 & 7.8 & 0.1 & \begin{tabular}{|r|}
12.9 \\
\end{tabular} & 8.9 & 72.6 & 59.7 & 10.5 & 9.9 & 11.9 & 5.0 & 196.3 \\
\hline & 2000 & 44.2 & 0.0 & 0.6 & 4.0 & 2.2 & 1.0 & 3.9 & 1.3 & 7.2 & 0.1 & 11.5 & 9.2 & 76.2 & 64.7 & 9.8 & 9.1 & 9.6 & 4.5 & 211.6 \\
\hline \multirow[t]{3}{*}{ Indonesia (IND) } & 1992 & 3.0 & 2.3 & 0.0 & 6.1 & 1.2 & 0.4 & 8.3 & 0.7 & 4.4 & 0.5 & 20.3 & 11.2 & 47.3 & 27.0 & 17.8 & 16.5 & 24.9 & 10.0 & 35.1 \\
\hline & 1996 & 2.6 & 2.8 & 0.0 & 5.1 & 2.2 & 0.6 & 7.7 & 1.5 & 3.9 & 0.6 & 21.4 & 12.7 & 48.7 & 27.3 & 16.6 & 15.3 & 24.5 & 10.3 & 53.5 \\
\hline & 2000 & 3.8 & 2.8 & 0.0 & 4.1 & 3.5 & 1.0 & 11.7 & 2.3 & 3.7 & 0.5 & 17.1 & 19.1 & 50.7 & 33.7 & 18.0 & 16.7 & 18.2 & 13.0 & 53.6 \\
\hline \multirow{3}{*}{ Korea, Rp of (KOR) } & 1992 & 2.9 & 5.1 & 2.0 & 0.0 & 1.2 & 0.6 & 3.6 & 1.1 & 2.4 & 0.3 & 21.5 & 8.8 & 40.7 & 19.2 & 28.6 & 26.1 & 16.2 & 14.4 & 114.8 \\
\hline & 1996 & 6.9 & 4.7 & 1.8 & 0.0 & 2.2 & 1.0 & 3.1 & 1.2 & 2.7 & 0.8 & 18.7 & 10.1 & 43.2 & 24.5 & 24.5 & 22.6 & 16.4 & 15.9 & 200.1 \\
\hline & 2000 & 9.4 & 3.9 & 1.5 & 0.0 & 2.6 & 1.8 & 3.3 & 1.2 & 4.7 & 0.7 & 16.8 & 11.1 & 46.0 & 29.2 & 26.8 & 24.5 & 14.9 & 12.4 & 241.4 \\
\hline \multirow[t]{3}{*}{ Malaysia (MAL) } & 1992 & 1.3 & 3.7 & 0.8 & 2.2 & 0.0 & 0.6 & 19.8 & 2.0 & 4.8 & 0.1 & 19.6 & 23.4 & 55.1 & 35.5 & 23.7 & 22.6 & 16.3 & 4.9 & 57.1 \\
\hline & 1996 & 2.0 & 4.7 & 1.4 & 3.4 & 0.0 & 1.1 & 17.6 & 2.8 & 4.9 & 0.2 & 19.5 & 23.1 & 57.7 & 38.2 & 21.2 & 20.2 & 15.6 & 5.5 & 119.8 \\
\hline & 2000 & 3.0 & 4.2 & 1.8 & 3.4 & 0.0 & 2.2 & 17.0 & 3.2 & 4.8 & 0.3 & 16.7 & 24.5 & 56.9 & 40.1 & 23.4 & 22.2 & 13.7 & 6.0 & 144.3 \\
\hline \multirow[t]{3}{*}{ Philippines (PHL) } & 1992 & 1.5 & 0.0 & 1.0 & 5.1 & 2.8 & 0.0 & 6.5 & 1.8 & 0.0 & 0.0 & 29.6 & 12.1 & 48.2 & 18.6 & 45.7 & 43.8 & 4.3 & 1.8 & 13.0 \\
\hline & 1996 & 1.3 & 5.0 & 0.9 & 4.2 & 2.9 & 0.0 & 6.1 & 2.7 & 4.8 & 0.3 & 23.3 & 12.9 & 51.5 & 28.2 & 29.2 & 28.4 & 15.2 & 4.1 & 42.2 \\
\hline & 2000 & 1.7 & 5.0 & 1.0 & 5.2 & 3.7 & 0.0 & 7.9 & 3.3 & 7.8 & 0.1 & 18.1 & 16.1 & 53.9 & 35.8 & 27.4 & 26.2 & 15.3 & 3.4 & 59.4 \\
\hline \multirow[t]{3}{*}{ Singapore (SGP) } & 1992 & 1.7 & 5.3 & 2.9 & 2.9 & 13.0 & 0.8 & 0.0 & 4.8 & 4.8 & 0.2 & 16.2 & 21.6 & 50.4 & 34.2 & 23.8 & 23.0 & 16.9 & 8.9 & 101.2 \\
\hline & 1996 & 2.7 & 5.5 & 0.0 & 3.4 & 17.0 & 1.5 & 0.0 & 5.8 & 4.3 & 0.5 & 14.4 & 24.9 & 56.2 & 41.8 & 20.9 & 20.2 & 15.2 & 7.7 & 209.3 \\
\hline & 2000 & 4.6 & 5.0 & 2.8 & 3.8 & 18.5 & 2.6 & 0.0 & 4.5 & 5.8 & 0.5 & 13.5 & 28.9 & 59.3 & 45.8 & 19.5 & 18.6 & 13.4 & 7.7 & 225.2 \\
\hline \multirow[t]{3}{*}{ Thailand (THA) } & 1992 & 1.4 & 3.2 & 0.4 & 2.7 & 2.3 & 0.5 & 8.3 & 0.0 & 4.5 & 0.1 & 26.5 & 11.6 & 50.2 & 23.8 & 20.4 & 19.2 & 20.0 & 9.3 & 49.0 \\
\hline & 1996 & 2.6 & 3.4 & 1.0 & 2.8 & 4.1 & 1.0 & 10.3 & 0.0 & 3.0 & 0.0 & 26.7 & 16.4 & 54.9 & 28.2 & 19.9 & 16.7 & 18.6 & 6.6 & 90.3 \\
\hline & 2000 & 4.7 & 3.6 & 1.6 & 2.9 & 5.0 & 2.1 & 7.9 & 0.0 & 4.8 & 0.9 & 21.5 & 17.4 & 55.7 & 34.2 & 20.1 & 18.9 & 15.1 & 9.1 & 94.7 \\
\hline \multirow[t]{3}{*}{ Taiwan (TWN) } & 1992 & 6.4 & 0.0 & 1.7 & 3.1 & 3.0 & 0.0 & 5.4 & 2.5 & 0.0 & 0.0 & 26.0 & 12.5 & 48.0 & 22.0 & 41.6 & 39.1 & 5.5 & 4.9 & 90.5 \\
\hline & 1996 & 9.4 & 8.7 & 1.2 & 3.1 & 3.3 & 1.2 & 5.2 & 1.6 & 0.0 & 0.0 & 18.7 & 12.4 & 52.3 & 33.6 & 25.9 & 15.3 & 15.3 & 6.5 & 175.1 \\
\hline & 2000 & 10.4 & 6.3 & 0.8 & 4.7 & 2.8 & 1.9 & 5.4 & 1.9 & 0.0 & 0.0 & 19.2 & 13.6 & 53.4 & 34.2 & 26.4 & 24.1 & 14.6 & 5.6 & 244.3 \\
\hline
\end{tabular}




\begin{tabular}{|c|c|c|c|c|c|c|c|c|c|c|c|c|c|c|c|c|c|c|c|c|}
\hline \multirow[t]{3}{*}{ Vietnam (VNM) } & 1992 & 3.9 & 0.0 & 12.3 & 24.4 & 2.3 & 0.0 & 13.2 & 2.7 & 0.0 & 0.0 & 33.9 & 30.4 & 92.7 & 58.8 & 0.7 & 0.1 & 3.3 & 3.3 & 1.5 \\
\hline & 1996 & 6.0 & 1.2 & 3.3 & 15.2 & 2.4 & 1.4 & 10.4 & 0.3 & 0.0 & 0.0 & 17.6 & 17.7 & 57.7 & 40.1 & 6.9 & 29.7 & 29.7 & 5.7 & 10.2 \\
\hline & 2000 & 8.1 & 1.1 & 2.0 & 11.5 & 3.2 & 0.6 & 7.3 & 5.6 & 0.0 & 0.0 & 19.9 & 19.1 & 59.3 & 39.4 & 4.5 & 3.4 & 30.5 & 5.7 & 1 \\
\hline \multirow[t]{3}{*}{ Japan (JPN) } & 1992 & 4.8 & 4.9 & 1.7 & 5.5 & 2.2 & 0.9 & 3.2 & 2.8 & 5.7 & 0.1 & 0.0 & 10.9 & 32.0 & 32.0 & 32.2 & 29.4 & 22.4 & 13.4 & 412.0 \\
\hline & 1996 & 8.7 & 4.3 & 2.2 & 6.3 & 3.6 & 1.9 & 4.3 & 3.9 & 5.9 & 0.3 & 0.0 & 16.3 & 41.4 & 41.4 & 30.2 & 28.3 & 18.3 & 10.1 & \\
\hline & 2000 & 10.8 & 4.0 & 1.8 & 6.0 & 3.4 & 2.3 & 3.6 & 2.9 & 7.3 & 0.4 & 0.0 & 14.4 & 42.5 & 42.5 & 31.2 & 28.9 & 17.5 & 8.8 & 644.3 \\
\hline
\end{tabular}

\section{(F) Total trade net of parts and components}

\begin{tabular}{|c|c|c|c|c|c|c|c|c|c|c|c|c|c|c|c|c|c|c|c|c|}
\hline & & $\mathrm{CHN}$ & HKG & IDN & KOR & MAL & PHL & SGP & THA & TWN & VNM & JPN & ASEAN & EAS & EAS-J & NAF & USA & $\mathrm{EU}$ & Other & WLD \\
\hline \multirow[t]{3}{*}{ China, PRC $(\mathrm{CHN})$} & 1992 & 0.0 & 41.4 & 0.8 & 2.2 & 0.4 & 0.2 & 1.0 & 0.4 & 4.3 & 0.0 & 13.2 & 2.9 & 64.1 & 51.0 & 12.8 & 11.6 & 12.3 & 10.7 & 109.6 \\
\hline & 1996 & 0.0 & 16.0 & 0.8 & 6.4 & 0.9 & 0.3 & 1.8 & 0.7 & 7.0 & 0.3 & 19.3 & 4.7 & 53.7 & 34.4 & 18.7 & 17.4 & 16.2 & 11.4 & 192.3 \\
\hline & 2000 & 0.0 & 12.8 & 1.2 & 6.4 & 1.0 & 0.4 & 1.8 & 0.9 & 6.2 & 0.4 & 17.3 & 5.7 & 48.6 & 31.3 & 21.6 & 19.9 & 16.8 & 13.0 & 290.8 \\
\hline \multirow[t]{3}{*}{ Hong Kong (HKG) } & 1992 & 47.8 & 0.0 & 0.8 & 4.5 & 1.3 & 0.0 & 3.5 & 1.3 & 0.0 & 0.0 & 17.1 & 6.9 & 76.3 & 59.2 & 15.1 & 13.5 & 3.3 & 5.3 & 94.8 \\
\hline & 1996 & 42.8 & 0.0 & 0.8 & 4.1 & 1.7 & 0.3 & 3.0 & 1.3 & 6.8 & 0.1 & 10.8 & 7.2 & 71.9 & 61.0 & 9.8 & 9.1 & 12.6 & 5.8 & 154.7 \\
\hline & 2000 & 50.9 & 0.0 & 0.7 & 3.7 & 1.4 & 0.5 & 2.1 & 1.2 & 5.2 & 0.1 & 8.9 & 6.1 & 74.9 & 66.0 & 9.2 & 8.6 & 10.2 & 5.7 & 152.5 \\
\hline \multirow[t]{3}{*}{ Indonesia (IND) } & 1992 & 3.4 & 2.4 & 0.0 & 6.4 & 1.2 & 0.4 & 8.4 & 0.7 & 4.3 & 0.6 & 18.5 & 11.3 & 46.4 & 27.9 & 17.8 & 16.5 & 25.0 & 10.8 & 30.5 \\
\hline & 1996 & 2.8 & 3.1 & 0.0 & 5.5 & 2.1 & 0.7 & 7.2 & 1.5 & 4.1 & 0.7 & 18.9 & 12.2 & 46.6 & 27.7 & 17.3 & 16.0 & 24.7 & 11.4 & 44.9 \\
\hline & 2000 & 4.1 & 3.0 & 0.0 & 4.5 & 3.0 & 1.0 & 9.9 & 2.3 & 3.8 & 0.6 & 14.6 & 16.8 & 47.0 & 32.3 & 18.9 & 17.5 & 19.6 & 14.6 & 45.0 \\
\hline \multirow[t]{3}{*}{ Korea, Rp of (KOR) } & 1992 & 3.4 & 4.7 & 2.3 & 0.0 & 0.7 & 0.6 & 1.5 & 1.1 & 2.1 & 0.4 & 19.8 & 6.6 & 36.7 & 16.9 & 28.3 & 25.4 & 17.7 & 17.3 & 90.1 \\
\hline & 1996 & 7.9 & 4.6 & 2.1 & 0.0 & 1.2 & 0.9 & 2.0 & 1.3 & 2.2 & 0.9 & 17.3 & 8.3 & 40.5 & 23.1 & 22.2 & 20.3 & 17.6 & 19.8 & 147.7 \\
\hline & 2000 & 10.4 & 3.6 & 1.9 & 0.0 & 1.2 & 1.0 & 2.1 & 1.0 & 2.8 & 0.9 & 15.7 & 8.1 & 40.7 & 25.0 & 26.2 & 23.7 & 16.6 & 16.5 & 159.8 \\
\hline \multirow[t]{3}{*}{ Malaysia (MAL) } & 1992 & 1.9 & 3.5 & 1.1 & 1.9 & 0.0 & 0.4 & 16.9 & 1.4 & 5.3 & 0.1 & 19.6 & 19.9 & 52.3 & 32.7 & 22.2 & 20.8 & 18.7 & 6.8 & 34.8 \\
\hline & 1996 & 2.8 & 4.8 & 1.8 & 2.4 & 0.0 & 0.6 & 12.6 & 2.0 & 4.6 & 0.3 & 20.4 & 17.3 & 52.7 & 32.3 & 20.9 & 19.5 & 18.6 & 7.9 & 65.8 \\
\hline & 2000 & 3.6 & 4.2 & 2.4 & 2.8 & 0.0 & 0.8 & 12.5 & 2.5 & 4.2 & 0.4 & 18.5 & 18.6 & 52.2 & 33.7 & 23.0 & 21.5 & 15.6 & 9.1 & 66.6 \\
\hline \multirow[t]{3}{*}{ Philippines (PHL) } & 1992 & 2.0 & 0.0 & 1.4 & 5.8 & 1.5 & 0.0 & 5.9 & 1.8 & 0.0 & 0.0 & 29.7 & 10.5 & 47.9 & 18.3 & 44.7 & 42.6 & 5.2 & 2.2 & 9.0 \\
\hline & 1996 & 2.1 & 5.3 & 1.5 & 5.3 & 1.5 & 0.0 & 4.4 & 1.6 & 5.9 & 0.5 & 21.5 & 9.5 & 49.6 & 28.1 & 28.6 & 27.3 & 15.4 & 6.4 & 22.3 \\
\hline & 2000 & 2.4 & 4.9 & 2.1 & 5.6 & 2.0 & 0.0 & 5.4 & 2.3 & 7.3 & 0.3 & 19.8 & 12.1 & 52.1 & 32.3 & 28.5 & 27.3 & 14.2 & 5.1 & 23.6 \\
\hline \multirow[t]{3}{*}{ Singapore (SGP) } & 1992 & 2.0 & 4.7 & 3.6 & 2.5 & 11.5 & 0.7 & 0.0 & 3.4 & 4.4 & 0.3 & 15.1 & 19.6 & 45.9 & 30.7 & 24.3 & 23.4 & 19.4 & 10.4 & 70.7 \\
\hline & 1996 & 3.0 & 5.5 & 0.0 & 3.0 & 13.8 & 1.1 & 0.0 & 5.9 & 3.9 & 0.7 & 14.1 & 21.5 & 52.6 & 38.5 & 20.5 & 19.8 & 16.8 & 10.1 & 123.0 \\
\hline & 2000 & 5.0 & 4.8 & 4.0 & 3.6 & 14.7 & 1.8 & 0.0 & 4.1 & 4.5 & 0.7 & 14.9 & 25.3 & 54.8 & 39.9 & 20.2 & 19.4 & 15.0 & 9.9 & 111.2 \\
\hline
\end{tabular}




\begin{tabular}{|l|r|r|r|r|r|r|r|r|r|r|r|r|r|r|r|r|r|r|r|r|}
\hline Thailand (THA) & 1992 & 1.6 & 3.4 & 0.5 & 2.8 & 1.3 & 0.4 & 4.6 & 0.0 & 4.5 & 0.1 & 25.1 & 6.9 & 45.0 & 19.9 & 21.0 & 19.6 & 22.5 & 11.5 & 37.1 \\
\hline & 1996 & 2.7 & 3.7 & 1.2 & 2.9 & 2.3 & 0.6 & 8.5 & 0.0 & 3.1 & 0.0 & 25.5 & 12.6 & 50.6 & 25.1 & 21.1 & 17.4 & 21.2 & 7.2 & 64.3 \\
\hline & 2000 & 4.4 & 3.8 & 2.0 & 2.6 & 3.4 & 1.1 & 5.3 & 0.0 & 4.4 & 0.8 & 21.0 & 12.6 & 50.0 & 29.0 & 20.7 & 19.5 & 17.2 & 12.1 & 59.0 \\
\hline & 1992 & 6.8 & 0.0 & 1.9 & 2.8 & 2.7 & 0.0 & 4.5 & 2.4 & 0.0 & 0.0 & 23.9 & 11.6 & 45.1 & 21.2 & 43.5 & 40.8 & 5.6 & 5.7 & 68.8 \\
\hline & 1996 & 11.1 & 8.8 & 1.5 & 2.7 & 2.5 & 1.1 & 4.0 & 1.7 & 0.0 & 0.0 & 17.6 & 10.7 & 50.9 & 33.3 & 25.2 & 16.4 & 16.4 & 7.5 & 121.0 \\
\hline & 2000 & 11.8 & 5.2 & 1.1 & 2.9 & 1.8 & 1.1 & 3.3 & 1.7 & 0.0 & 0.0 & 20.4 & 10.3 & 49.5 & 29.1 & 27.9 & 25.6 & 15.7 & 6.9 & 152.3 \\
\hline & 1992 & 3.7 & 0.0 & 12.8 & 24.5 & 2.3 & 0.0 & 12.8 & 2.6 & 0.0 & 0.0 & 34.5 & 30.5 & 93.2 & 58.7 & 0.7 & 0.1 & 2.9 & 3.2 & 1.4 \\
\hline & 1996 & 6.0 & 1.2 & 3.5 & 14.9 & 2.0 & 1.2 & 9.4 & 0.2 & 0.0 & 0.0 & 18.4 & 16.2 & 56.6 & 38.3 & 7.0 & 30.7 & 30.7 & 5.6 & 9.3 \\
\hline & 2000 & 8.7 & 1.1 & 2.1 & 11.6 & 2.4 & 0.6 & 6.2 & 3.8 & 0.0 & 0.0 & 19.1 & 15.7 & 55.7 & 36.6 & 4.3 & 3.1 & 33.9 & 6.1 & 12.3 \\
\hline & 1992 & 5.6 & 5.0 & 1.7 & 5.1 & 1.7 & 0.8 & 2.6 & 2.6 & 5.1 & 0.2 & 0.0 & 9.6 & 30.5 & 30.5 & 30.6 & 27.9 & 23.8 & 15.2 & 325.1 \\
\hline & 1996 & 10.0 & 4.1 & 2.3 & 5.8 & 2.9 & 1.5 & 3.5 & 3.7 & 5.2 & 0.4 & 0.0 & 14.3 & 39.5 & 39.5 & 28.4 & 26.7 & 20.0 & 12.1 & 411.3 \\
\hline & 2000 & 11.9 & 3.4 & 1.7 & 5.4 & 2.6 & 1.5 & 2.8 & 2.6 & 6.8 & 0.5 & 0.0 & 11.7 & 39.3 & 39.3 & 31.0 & 28.9 & 19.1 & 10.6 & 460.6 \\
\hline
\end{tabular}


Table A-2: East Asia: Share of Parts and Components in Bilateral Manufacturing Trade Flows, 1992, 1996 and 2000

\section{(A) Manufacturing Exports}

\begin{tabular}{|c|c|c|c|c|c|c|c|c|c|c|c|c|c|c|c|c|c|c|c|}
\hline \multirow[t]{2}{*}{ Exporter } & & \multicolumn{18}{|c|}{ Partner } \\
\hline & & $\mathrm{CHN}$ & $\mathrm{HKG}$ & IDN & KOR & MAL & PHL & SGP & THA & TWN & VNM & JPN & ASEAN & EAS & EAS-J & NAF & USA & EUU & WLD \\
\hline \multirow[t]{3}{*}{ China, PRC (CHN) } & 1992 & --- & 8.1 & 9.8 & 6.9 & 10.3 & 8.3 & 8.9 & 4.6 & 19.8 & 5.5 & 6.2 & 8.2 & 7.9 & 8.2 & 4.1 & 4.3 & 2.9 & \\
\hline & 1996 & --- & 11.2 & 16.7 & 17.1 & 24.7 & 14.5 & 19.8 & 11.6 & 25.5 & 8.0 & 11.8 & 17.7 & 12.9 & 13.5 & 7.1 & 7.2 & 6.0 & \\
\hline & 2000 & --- & 21.4 & 15.8 & 21.6 & 29.9 & 33.1 & 37.0 & 33.9 & 28.4 & 6.7 & 15.4 & 29.0 & 20.6 & 23.2 & 9.4 & 9.1 & 10.9 & 14. \\
\hline \multirow[t]{3}{*}{ Hong Kong (HKG) } & 1992 & 24.8 & --- & 14.9 & 78.1 & 31.6 & --- & 47.1 & 24.7 & --- & --- & 21.7 & 38.9 & 28.3 & 28.7 & 12.0 & 12.2 & 7.9 & 21 \\
\hline & 1996 & 36.2 & -- & 19.5 & 39.4 & 34.4 & 37.9 & 72.1 & 35.2 & 34.1 & 8.3 & 26.3 & 52.4 & 38.2 & 39.6 & 16.3 & 16.3 & 16.3 & 26 \\
\hline & 2000 & 34.6 & --- & 11.8 & 40.8 & 56.7 & 39.2 & 70.4 & 27.0 & 42.0 & 25.0 & 44.9 & 52.7 & 38.5 & 38.1 & 13.6 & 13.7 & 19.4 & 25 \\
\hline \multirow[t]{3}{*}{ Indonesia (IND) } & 1992 & 0.0 & 4.6 & --- & 1.3 & 5.4 & 2.0 & 8.0 & 17.3 & 4.5 & 0.0 & 5.3 & 7.6 & 5.3 & 5.3 & 3.2 & 3.4 & 2.6 & 4. \\
\hline & 1996 & 1.1 & 7.2 & --- & 5.2 & 21.5 & 4.6 & 23.2 & 14.3 & 4.5 & 1.8 & 7.2 & 19.5 & 11.2 & 13.4 & 4.4 & 4.3 & 4.0 & 7. \\
\hline & 2000 & 5.7 & 12.7 & --- & 10.4 & 35.3 & 18.2 & 34.8 & 20.0 & 12.2 & 5.8 & 20.8 & 31.4 & 23.0 & 23.9 & 9.1 & 9.4 & 6.7 & 14. \\
\hline \multirow[t]{3}{*}{ Korea, Rp of (KOR) } & 1992 & 8.1 & 19.6 & 10.0 & --- & 57.2 & 17.5 & 66.1 & 19.7 & 29.4 & 3.7 & 18.4 & 38.8 & 25.1 & 28.4 & 19.5 & 20.4 & 13.6 & 17. \\
\hline & 1996 & 13.1 & 24.7 & 16.8 & --- & 69.1 & 25.5 & 58.7 & 21.2 & 34.2 & 10.9 & 32.5 & 40.3 & 29.9 & 29.1 & 36.8 & 37.7 & 23.8 & 25 \\
\hline & 2000 & 26.7 & 35.4 & 21.1 & --- & 66.8 & 57.0 & 66.6 & 40.8 & 58.1 & 13.4 & 39.7 & 50.0 & 40.9 & 41.3 & 30.2 & 30.5 & 26.2 & 30. \\
\hline \multirow[t]{3}{*}{ Malaysia (MAL) } & 1992 & 6.4 & 49.7 & 28.4 & 72.0 & --- & 34.1 & 45.2 & 56.5 & 53.9 & 6.5 & 45.5 & 45.2 & 45.7 & 45.8 & 42.3 & 43.6 & 32.6 & 40. \\
\hline & 1996 & 16.1 & 42.5 & 27.9 & 35.0 & --- & 42.5 & 59.6 & 61.6 & 64.6 & 20.6 & 35.2 & 57.1 & 49.0 & 52.6 & 39.9 & 40.9 & 35.8 & 42. \\
\hline & 2000 & 50.6 & 52.5 & 27.4 & 60.5 & --- & 65.4 & 68.1 & 67.4 & 61.6 & 27.8 & 40.5 & 65.2 & 57.0 & 61.7 & 47.2 & 47.6 & 44.0 & 49. \\
\hline \multirow[t]{3}{*}{ Philippines (PHL) } & 1992 & 1.8 & --- & 10.3 & 52.1 & 90.6 & --- & 66.3 & 25.7 & --- & --- & 27.0 & 64.4 & 41.9 & 58.1 & 25.9 & 26.0 & 14.8 & 29. \\
\hline & 1996 & 17.6 & 56.3 & 26.7 & 67.0 & 92.1 & --- & 82.2 & 91.9 & 66.4 & 2.9 & 48.9 & 82.5 & 64.2 & 74.1 & 42.7 & 43.4 & 56.0 & 52. \\
\hline & 2000 & 81.8 & 79.3 & 52.7 & 77.2 & 93.8 & --- & 85.9 & 92.0 & 72.1 & 14.5 & 52.8 & 87.5 & 72.9 & 81.4 & 52.5 & 52.0 & 66.5 & 64. \\
\hline \multirow[t]{3}{*}{ Singapore (SGP) } & 1992 & 23.1 & 31.3 & 22.3 & 31.5 & 39.5 & 25.5 & --- & 43.4 & 39.5 & 6.7 & 37.6 & 37.6 & 35.6 & 35.3 & 27.5 & 28.1 & 19.3 & 28.2 \\
\hline & 1996 & 41.9 & 38.2 & --- & 37.2 & 53.1 & 42.6 & --- & 41.3 & 49.3 & 16.8 & 37.9 & 48.6 & 43.3 & 44.2 & 38.4 & 38.5 & 38.8 & 39. \\
\hline & 2000 & 50.3 & 55.2 & 13.5 & 49.7 & 63.3 & 47.3 & --- & 51.3 & 58.0 & 27.8 & 41.1 & 56.3 & 54.1 & 56.2 & 47.0 & 46.4 & 47.7 & 49. \\
\hline
\end{tabular}




\begin{tabular}{|c|c|c|c|c|c|c|c|c|c|c|c|c|c|c|c|c|c|c|c|}
\hline Thailand (THA) & 1992 & 6.8 & 15.6 & 12.9 & 29.4 & 55.5 & 38.1 & 61.4 & --- & 24.7 & 5.4 & 25.9 & 57.2 & 35.9 & 41.9 & 16.6 & 17.3 & 11.7 & 21.2 \\
\hline & 1996 & 29.2 & 17.7 & 20.3 & 33.3 & 60.0 & 29.5 & 40.0 & --- & --- & $\ldots$ & 20.0 & 42.3 & 31.2 & 36.6 & 16.5 & 16.4 & 16.4 & 23. \\
\hline & 2000 & 54.0 & 33.3 & 22.4 & 61.1 & 57.4 & 49.5 & 69.2 & --- & 60.9 & 22.6 & 37.6 & 56.9 & 47.4 & 51.6 & 26.8 & 26.3 & 32.2 & 35. \\
\hline \multirow[t]{3}{*}{ Taiwan (TWN) } & 1992 & 18.4 & 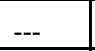 & 18.3 & 32.4 & 26.0 & $-{ }_{-1}$ & 32.7 & 24.3 & $-\ldots$ & $-\ldots$ & 19.1 & 26.8 & 22.6 & 24.0 & 18.6 & 18.6 & 21.3 & 20.1 \\
\hline & 1996 & 17.1 & 30.2 & 16.4 & 45.9 & 36.5 & 22.7 & 45.0 & 27.2 & $-\ldots$ & $-\ldots$ & 27.6 & 34.1 & 27.9 & 28.0 & 32.9 & 27.2 & 27.2 & 28. \\
\hline & 2000 & 29.8 & 48.2 & 12.9 & 64.5 & 58.1 & 50.0 & 66.6 & 30.5 & $-\ldots$ & - & 41.6 & 48.9 & 43.0 & 43.4 & 33.2 & 32.8 & 35.0 & 37.8 \\
\hline \multirow[t]{3}{*}{ Vietnam (VNM) } & 1992 & 33.3 & $\ldots$ & \begin{tabular}{l|}
-- \\
--
\end{tabular} & 5.9 & 0.0 & --- & 0.0 & 0.0 & --- & $-\ldots$ & 0.9 & 0.0 & 2.7 & 9.1 & 0.0 & --- & 0.0 & \\
\hline & 1996 & 27.3 & 14.1 & 66.7 & 10.4 & 52.9 & 79.4 & 29.1 & 46.2 & $-\ldots$ & $-\ldots$ & 2.9 & 44.4 & 12.0 & 28.7 & 0.0 & 0.5 & 0.5 & 5.2 \\
\hline & 2000 & 13.0 & 11.9 & 50.0 & 13.7 & 60.5 & 14.3 & 18.5 & 88.8 & -- & $-\ldots$ & 10.8 & 56.5 & 22.5 & 40.5 & 0.8 & 0.8 & 1.0 & 8.7 \\
\hline \multirow[t]{3}{*}{ Japan (JPN) } & 1992 & 11.7 & 19.8 & 24.1 & 31.4 & 41.7 & 32.0 & 35.9 & 30.6 & 33.7 & 2.5 & --- & 33.4 & 28.3 & 28.3 & 25.6 & 25.7 & 19.3 & 22.5 \\
\hline & 1996 & 23.5 & 28.6 & 30.4 & 30.8 & 49.8 & 46.8 & 42.8 & 34.4 & 38.7 & 7.4 & $-\ldots$ & 40.6 & 34.9 & 34.9 & 32.4 & 31.9 & 26.5 & 30.2 \\
\hline & 2000 & 32.8 & 38.5 & 44.6 & 34.9 & 51.5 & 54.8 & 45.2 & 42.3 & 30.0 & 24.6 & --- & 46.8 & 38.4 & 38.4 & 28.2 & 27.5 & 27.6 & 3 \\
\hline
\end{tabular}

\section{(B ) Manufacturing Imports}

\begin{tabular}{|c|c|c|c|c|c|c|c|c|c|c|c|c|c|c|c|c|c|c|c|}
\hline & & $\mathrm{CHN}$ & HKG & IDN & KOR & MAL & PHL & SGP & THA & TWN & VNM & JPN & ASEAN & EAS & EAS-J & NAF & USA & EUU & WLD \\
\hline \multirow[t]{3}{*}{ China, PRC (CHN) } & 1992 & --- & 24.8 & 0.2 & 11.9 & 6.9 & 1.8 & 20.7 & 8.9 & 18.4 & 33.3 & 20.5 & 7.2 & 21.4 & 21.7 & 15.3 & 16.0 & 21.2 & 19.5 \\
\hline & 1996 & --- & 27.9 & 2.3 & 15.3 & 25.3 & 50.5 & 42.7 & 29.2 & 17.1 & 27.3 & 29.4 & 30.0 & 24.0 & 20.4 & $\begin{array}{l}17.3 \\
\end{array}$ & $\begin{array}{r}17.3 \\
\end{array}$ & 17.6 & 21.1 \\
\hline & 2000 & --- & 35.3 & 15.6 & 27.4 & 60.2 & 77.5 & 41.5 & 44.9 & 29.8 & 13.0 & 37.3 & 47.2 & 35.1 & 33.8 & 28.2 & 25.7 & 34.4 & 33.5 \\
\hline \multirow[t]{3}{*}{ Hong Kong (HKG) } & 1992 & 8.1 & --- & 4.6 & 19.6 & 49.7 & --- & 31.3 & 15.6 & --- & --- & 19.8 & 29.9 & 14.5 & 12.3 & 25.8 & 26.0 & 16.9 & 15.1 \\
\hline & 1996 & 10.1 & -- & 6.3 & 25.3 & 35.7 & 59.4 & 40.0 & 17.7 & 30.2 & 14.1 & 34.2 & 33.9 & 20.5 & 17.4 & 32.7 & 33.5 & 16.8 & 20.4 \\
\hline & 2000 & 15.7 & --- & 8.4 & 34.8 & 54.3 & 68.9 & 59.9 & 33.7 & 48.2 & 11.9 & 43.9 & 51.9 & 28.5 & 25.7 & 42.8 & 42.1 & 23.8 & 28.2 \\
\hline \multirow[t]{3}{*}{ Indonesia (IND) } & 1992 & 8.3 & 14.9 & --- & 11.4 & 24.4 & 10.3 & 22.3 & 16.7 & 18.3 & --- & 26.7 & 21.8 & 21.5 & 15.5 & 22.2 & 22.5 & 19.4 & 20.5 \\
\hline & 1996 & 13.4 & 15.6 & --- & 12.7 & 21.7 & 14.1 & 18.3 & 20.3 & 16.4 & 66.7 & 36.3 & 19.5 & 26.6 & 16.0 & 22.1 & 22.6 & 22.2 & 23.8 \\
\hline & 2000 & 11.5 & 10.4 & --- & 7.1 & 13.8 & 32.1 & 13.5 & 12.3 & 12.9 & 50.0 & 35.4 & 14.0 & 21.3 & 11.9 & 20.4 & 19.0 & 15.2 & 19.4 \\
\hline \multirow[t]{3}{*}{ Korea, Rp of (KOR) } & 1992 & 5.3 & 78.1 & 1.6 & --- & 48.5 & 52.1 & 70.3 & 30.0 & 32.4 & 5.9 & 32.1 & 47.8 & 33.5 & 37.3 & 27.2 & 27.8 & 15.2 & 26.7 \\
\hline & 1996 & 18.9 & 60.8 & 5.0 & --- & 43.1 & 76.5 & 40.1 & 33.3 & 45.9 & 10.4 & 31.2 & 36.0 & 32.0 & 33.6 & 29.7 & 30.5 & 19.0 & 27.4 \\
\hline & 2000 & 26.9 & 66.6 & 10.4 & --- & 71.7 & 78.1 & 42.3 & 48.8 & 64.5 & 13.7 & 37.3 & 54.4 & 41.9 & 47.2 & 43.4 & 44.4 & 26.1 & 38.9 \\
\hline \multirow[t]{3}{*}{ Malaysia (MAL) } & 1992 & 11.1 & 31.6 & 3.8 & 41.5 & --- & 90.6 & 52.1 & 58.4 & 26.0 & 0.0 & 37.7 & 51.5 & 39.8 & 41.8 & 43.3 & 44.2 & 27.7 & 37.9 \\
\hline & 1996 & 24.4 & 44.9 & 28.9 & 68.6 & --- & 91.1 & 62.5 & 60.0 & 36.5 & 52.9 & 45.5 & 61.3 & 50.6 & 54.7 & 53.4 & 55.0 & 33.6 & 47.5 \\
\hline & 2000 & 41.8 & 58.0 & 44.5 & 63.1 & --- & 93.5 & 63.0 & 61.2 & 58.1 & 60.5 & 53.8 & 64.7 & 58.3 & 60.9 & 66.1 & 67.1 & 52.4 & 58.8 \\
\hline
\end{tabular}




\begin{tabular}{|c|c|c|c|c|c|c|c|c|c|c|c|c|c|c|c|c|c|c|c|}
\hline Philippines (PHL) & 1992 & 8.3 & --- & 2.0 & 17.5 & 34.1 & --- & 25.5 & 38.1 & --- & --- & 32.0 & 26.3 & 28.4 & 22.0 & 46.4 & 47.4 & 15.9 & 32.6 \\
\hline & 1996 & 17.0 & 36.6 & 8.2 & 29.8 & 49.1 & --- & 46.6 & 29.5 & 22.7 & 79.4 & 52.2 & 40.8 & 42.1 & 32.9 & 54.5 & 55.5 & 38.1 & 43. \\
\hline & 2000 & 19.4 & 34.8 & 7.8 & 48.3 & 56.5 & --- & 52.5 & 45.6 & 50.0 & 14.3 & 59.6 & 46.6 & 50.2 & 44.5 & 73.0 & 73.7 & 55.4 & 55. \\
\hline \multirow[t]{3}{*}{ Singapore (SGP) } & 1992 & 11.1 & 47.1 & 8.0 & 41.8 & 36.5 & 66.3 & --- & 59.1 & 32.7 & 0.0 & 34.0 & 36.3 & 37.0 & 39.4 & 30.2 & 30.5 & 21.1 & 32. \\
\hline & 1996 & 28.3 & 46.5 & --- & 56.6 & 51.6 & 82.8 & --- & 40.0 & 45.0 & 29.1 & 43.9 & 50.1 & 46.4 & 47.8 & 46.4 & 46.6 & 32.3 & 42.8 \\
\hline & 2000 & 42.3 & 46.3 & 34.8 & 59.2 & 58.1 & 83.0 & --- & 59.0 & 66.6 & 18.5 & 47.4 & 57.3 & 54.6 & 57.8 & 51.6 & 51.3 & 40.8 & 51.7 \\
\hline \multirow[t]{3}{*}{ Thailand (THA) } & 1992 & 7.6 & 24.7 & 8.2 & 19.7 & 60.0 & 25.7 & 53.2 & --- & 24.3 & 0.0 & 28.7 & 51.5 & 30.3 & 32.6 & 30.4 & 31.1 & 17.5 & 26. \\
\hline & 1996 & 20.8 & 31.5 & 11.6 & 24.6 & 60.3 & 82.7 & 44.2 & --- & 27.2 & 46.2 & 36.2 & 49.9 & 36.4 & 36.7 & 34.6 & 35.4 & 21.1 & 32. \\
\hline & 2000 & 33.8 & 35.6 & 26.3 & 34.2 & 57.5 & 82.6 & 41.9 & --- & 30.5 & 88.8 & 40.0 & 52.7 & 41.4 & 42.6 & 53.2 & 53.7 & 24.1 & 39.8 \\
\hline \multirow[t]{3}{*}{ Taiwan (TWN) } & 1992 & 19.8 & --- & 4.5 & 29.4 & 53.9 & --- & 39.5 & 24.7 & --- & --- & 33.7 & 36.2 & 33.5 & 33.1 & 25.2 & 25.4 & 23.3 & 29. \\
\hline & 1996 & 25.5 & 34.1 & 4.5 & 34.2 & 64.6 & 66.4 & 49.3 & --- & --- & --- & 38.7 & 51.7 & 40.4 & 43.1 & 32.9 & 22.8 & 22.8 & 35 \\
\hline & 2000 & 28.4 & 42.0 & 12.2 & 58.1 & 61.6 & 72.1 & 58.0 & 60.9 & --- & --- & 30.0 & 56.3 & 41.2 & 54.1 & 35.9 & 36.1 & 29.0 & \\
\hline \multirow[t]{3}{*}{ Vietnam (VNM) } & 1992 & 5.5 & --- & 0.0 & 3.7 & 6.5 & --- & 6.7 & 5.4 & --- & --- & 2.5 & 3.8 & 3.5 & 3.9 & 12.5 & 0.0 & 35.0 & 4.2 \\
\hline & 1996 & 8.0 & 8.3 & 1.8 & 10.9 & 20.6 & 2.9 & 16.8 & -- & --- & --- & 7.4 & 13.3 & 10.6 & 11.5 & 9.9 & 12.7 & 12.7 & 11 \\
\hline & 2000 & 6.7 & 25.0 & 5.8 & 13.4 & 27.8 & 14.5 & 27.8 & 22.6 & --- & --- & 24.6 & 22.9 & 18.4 & 16.4 & 36.7 & 40.4 & 19.0 & \\
\hline \multirow[t]{3}{*}{ Japan (JPN) } & 1992 & 4.5 & 21.7 & 4.3 & 17.7 & 28.3 & 27.0 & 32.1 & 24.4 & 19.1 & 0.9 & --- & 22.1 & 15.4 & 15.4 & 23.5 & 23.7 & 8.5 & 15 \\
\hline & 1996 & 9.9 & 28.5 & 9.1 & 36.6 & 22.3 & 39.3 & 32.9 & 20.0 & 27.6 & 2.9 & --- & 23.2 & 20.6 & 20.6 & 27.6 & 28.1 & 9.4 & 19 \\
\hline & 2000 & 13.7 & 46.2 & 17.1 & 35.9 & 34.1 & 51.2 & 41.8 & 24.5 & 41.6 & 10.8 & --- & 32.8 & 26.8 & 26.8 & 30.7 & 31.1 & 11.6 & \\
\hline
\end{tabular}


Table A-3: East Asia: Growth of Bilateral Manufacturing Trade Flow and the Contribution Parts and Components to Trade growth, 1992-2000 (\%)

(A) Growth of Manufacturing Exports

\begin{tabular}{|c|c|c|c|c|c|c|c|c|c|c|c|c|c|c|c|c|c|c|}
\hline \multirow[t]{2}{*}{ Exporter } & \multicolumn{18}{|c|}{ Partner } \\
\hline & $\mathrm{CHN}$ & HKG & IDN & KOR & MAL & PHL & SGP & THA & TWN & VNM & JPN & ASEAN & EAS & EAS-J & NAF & USA & EUU & WLD \\
\hline $\mathrm{CHN}$ & --- & 0.9 & \begin{tabular}{ll|}
12.4 \\
\end{tabular} & 11.8 & 10.4 & \begin{tabular}{|l|}
12.6 \\
\end{tabular} & \begin{tabular}{|l|}
9.4 \\
\end{tabular} & 8.0 & \begin{tabular}{|r|}
12.9 \\
\end{tabular} & $\begin{array}{l}17.6 \\
\end{array}$ & $\begin{array}{r}9.6 \\
\end{array}$ & $\begin{array}{r}10.4 \\
\end{array}$ & \begin{tabular}{|r|}
4.4 \\
\end{tabular} & 2.9 & \begin{tabular}{|l|}
10.9 \\
\end{tabular} & $\begin{array}{l}10.9 \\
\end{array}$ & 9.8 & 6.7 \\
\hline HKG & -5.5 & --- & -4.2 & -7.6 & -4.7 & --- & -6.6 & -5.9 & --- & --- & -5.4 & -4.7 & -4.9 & -4.9 & -2.2 & -1.8 & 3.0 & -3.2 \\
\hline IDN & 2.8 & 3.9 & --- & 2.0 & 10.2 & 8.7 & 4.2 & 8.0 & 3.5 & 2.2 & 4.6 & 5.3 & 4.5 & 4.5 & 4.8 & 4.8 & 3.4 & 4.4 \\
\hline KOR & 12.5 & 2.7 & 2.9 & --- & 6.5 & 9.0 & 3.1 & 2.6 & 8.1 & 8.1 & 3.0 & 4.6 & 5.2 & 6.1 & 3.9 & 4.0 & 4.8 & 4.5 \\
\hline MAL & 11.7 & 6.2 & 10.3 & 11.3 & --- & 11.3 & 4.6 & 8.4 & 8.2 & 13.8 & 8.4 & 5.5 & 6.7 & 6.2 & 5.7 & 5.6 & 5.2 & 6.2 \\
\hline PHL & 11.5 & --- & 8.5 & 14.9 & 11.0 & --- & 14.2 & 14.1 & --- & --- & 10.5 & 13.1 & 13.9 & 16.3 & 5.9 & 5.8 & 18.5 & 10.2 \\
\hline SGP & 11.4 & 4.9 & 4.3 & 8.9 & 7.6 & 8.6 & --- & 4.0 & 6.7 & 8.8 & 6.7 & 6.7 & 6.7 & 6.7 & 3.4 & 3.3 & 3.5 & 5.0 \\
\hline THA & 19.6 & 4.7 & 12.5 & 8.9 & 7.8 & 11.1 & 3.6 & --- & 8.9 & 16.1 & 4.7 & 5.9 & 6.0 & 6.7 & 4.0 & 4.0 & 3.7 & 4.8 \\
\hline TWN & 7.9 & --- & 0.1 & 7.8 & 3.8 & --- & 4.3 & 1.9 & --- & --- & 5.7 & 4.3 & 7.3 & 7.8 & 2.8 & 2.6 & 10.6 & 5.8 \\
\hline VNM & 18.6 & --- & --- & 13.7 & 20.8 & --- & 19.3 & 26.6 & --- & --- & 14.4 & 23.2 & 16.0 & 19.7 & 29.7 & --- & 29.6 & 21.0 \\
\hline JPN & 5.4 & 1.4 & 1.8 & 2.8 & 2.9 & 6.1 & 2.6 & 1.5 & 3.1 & 7.5 & --- & 2.8 & 2.9 & 2.9 & 2.0 & 2.1 & 0.7 & 1.8 \\
\hline \multicolumn{19}{|c|}{ (B) Contribution of parts and components to export growth } \\
\hline & $\mathrm{CHN}$ & \begin{tabular}{l|l} 
HKG & IL \\
\end{tabular} & $\mathrm{DN}$ & KOR & MAL & PHL & SGP & THA & TWN & VNM & JPN & ASEAN & EAS & EAS-J & NAF & USA & EUU & WLD \\
\hline $\mathrm{CHN}$ & --- & 100.0 & 16.6 & 23.8 & 33.7 & 36.3 & \begin{tabular}{|l|}
43.6 \\
\end{tabular} & 43.3 & \begin{tabular}{|r|}
29.4 \\
\end{tabular} & 6.8 & \begin{tabular}{|r|}
17.4 \\
\end{tabular} & \begin{tabular}{|r|}
32.9 \\
\end{tabular} & \begin{tabular}{|l|}
31.1 \\
\end{tabular} & $\begin{array}{r}44.7 \\
\end{array}$ & \begin{tabular}{|l|}
10.3 \\
\end{tabular} & $\begin{array}{r}9.9 \\
\end{array}$ & 12.6 & 17.9 \\
\hline HKG & 19.3 & \begin{tabular}{l|l|}
--- \\
\end{tabular} & 17.5 & 89.3 & 13.8 & 39.2 & 37.8 & 23.5 & 42.0 & 25.0 & 8.8 & 29.2 & 21.6 & 22.6 & 8.8 & 8.4 & 34.9 & 16.3 \\
\hline IDN & 14.4 & 20.4 & --- & 30.7 & 41.4 & 22.6 & 58.0 & 20.9 & 20.8 & 17.8 & 32.9 & 46.3 & 37.1 & 38.8 & 13.3 & 13.8 & 11.4 & 22.8 \\
\hline$\overline{\mathrm{KOR}}$ & 29.1 & 60.1 & 37.1 & --- & 71.2 & 67.1 & 67.3 & 75.2 & 67.1 & 16.4 & 69.4 & 58.9 & 51.2 & 47.9 & 40.6 & 40.0 & 35.4 & 41.0 \\
\hline MAL & 57.2 & 53.9 & 27.2 & 58.6 & --- & 70.5 & 85.7 & 70.6 & 64.0 & 29.9 & 39.1 & 77.1 & 61.9 & 69.5 & 49.9 & 49.8 & 51.4 & 54.4 \\
\hline PHL & 94.2 & 79.3 & 64.7 & 79.3 & 94.3 & --- & 87.8 & 98.4 & 72.1 & 14.5 & 57.7 & 90.2 & 76.0 & 82.9 & 66.8 & 66.1 & 68.9 & 71.0 \\
\hline SGP & 54.7 & 72.0 & 5.9 & 54.4 & 71.7 & 53.5 & --- & 58.6 & 66.1 & 33.4 & 42.6 & 64.4 & 62.2 & 65.3 & 69.9 & 68.8 & 80.1 & 64.5 \\
\hline THA & 55.8 & 46.6 & 23.6 & 69.5 & 58.1 & 51.4 & 77.8 & --- & 70.4 & 23.8 & 46.3 & 56.8 & 53.4 & 55.8 & 36.3 & 34.9 & 53.4 & 46.6 \\
\hline TWN & 33.5 & 48.2 & -207.7 & 75.2 & 90.7 & 50.0 & 95.5 & 45.0 & --- & --- & 54.3 & 67.6 & 50.8 & 49.9 & 54.9 & 56.4 & 37.5 & 47.4 \\
\hline VNM & 12.1 & 11.9 & \begin{tabular}{l|l|}
50.0 \\
\end{tabular} & 14.5 & 62.4 & 14.3 & 19.2 & 90.0 & --- & --- & 11.7 & 57.7 & 23.9 & 41.7 & 0.8 & 0.8 & 1.0 & 8.9 \\
\hline JPN & 45.5 & 101.3 & 96.6 & 40.2 & 65.6 & 66.2 & \begin{tabular}{|c|}
60.9 \\
\end{tabular} & 78.7 & 25.1 & 32.5 & --- & 66.9 & 52.7 & 52.7 & 34.1 & 31.2 & 86.2 & 50.1 \\
\hline
\end{tabular}




\begin{tabular}{|c|c|c|c|c|c|c|c|c|c|c|c|c|c|c|c|c|c|c|}
\hline \multicolumn{19}{|c|}{ (c) Growth of manufacturing Imports } \\
\hline & $\mathrm{CHN}$ & HKG & IDN & KOR & MAL & PHL & SGP & THA & TWN & VNM & JPN & ASEAN & EAS & EAS-J & NAF & USA & EUU & WLD \\
\hline $\mathrm{CHN}$ & --- & -4.0 & 6.0 & 13.7 & 17.1 & 18.9 & 14.1 & 20.1 & 7.9 & 18.6 & 6.6 & 13.5 & 5.3 & 4.7 & 5.4 & 5.3 & 5.9 & 5.5 \\
\hline HKG & 5.2 & \begin{tabular}{|l|}
--- \\
\end{tabular} & 3.6 & 2.8 & 6.8 & $\begin{array}{l}--- \\
\end{array}$ & 4.7 & 5.0 & --- & --- & 1.3 & 5.8 & 4.8 & 5.8 & 4.4 & 4.2 & 15.7 & 5.2 \\
\hline IDN & 4.6 & 2.4 & $\begin{array}{l}-- \\
\end{array}$ & \begin{tabular}{l|l}
-0.6 \\
\end{tabular} & 5.7 & 5.9 & 4.3 & 9.8 & 0.1 & --- & \begin{tabular}{c|}
-0.6 \\
\end{tabular} & 5.4 & 0.9 & 2.3 & -1.3 & -1.4 & -2.4 & -0.1 \\
\hline KOR & 9.0 & 2.0 & 2.0 & --- & 12.8 & 17.9 & 4.4 & 9.5 & 7.8 & 13.7 & 2.6 & 7.4 & 4.4 & 7.5 & 3.5 & 3.4 & 2.0 & 3.6 \\
\hline MAL & 9.4 & 5.5 & 9.5 & 6.4 & --- & 13.3 & 3.8 & 7.5 & 3.8 & 20.8 & 2.8 & 5.4 & 4.3 & 5.5 & 4.3 & 4.3 & 2.9 & 4.2 \\
\hline PHL & 8.5 & \begin{tabular}{l|l}
--- \\
\end{tabular} & 8.6 & 7.3 & 9.3 & --- & 6.3 & 10.3 & --- & --- & 3.6 & 7.9 & 6.7 & 10.0 & 5.0 & 5.1 & 13.3 & 6.9 \\
\hline SGP & 9.5 & 2.7 & 4.2 & 4.1 & 5.5 & 15.0 & --- & 4.1 & 4.3 & 19.3 & 2.5 & 5.6 & 4.4 & 5.5 & 3.2 & 3.2 & 2.7 & 3.9 \\
\hline THA & 8.7 & 3.1 & 10.1 & 2.6 & 8.2 & 13.5 & 3.1 & --- & 1.9 & 26.6 & 1.6 & 6.1 & 3.1 & 4.8 & 2.7 & 2.9 & 0.1 & 2.6 \\
\hline TWN & 12.9 & --- & 3.5 & 8.1 & 8.2 & --- & 6.7 & 8.9 & --- & --- & 3.1 & 8.3 & 5.0 & 8.7 & 3.3 & 3.3 & 13.4 & 5.1 \\
\hline VNM & 17.6 & --- & 2.2 & 8.1 & 13.8 & --- & 8.8 & 16.1 & --- & --- & 7.5 & 9.0 & 9.2 & 9.8 & 21.3 & 35.1 & 23.2 & 10.2 \\
\hline JPN & 8.3 & -1.2 & 4.6 & 3.1 & 9.5 & 11.5 & 6.1 & 4.7 & 5.7 & 14.4 & --- & 7.1 & 6.4 & 6.4 & 3.1 & 3.0 & 1.9 & 4.1 \\
\hline
\end{tabular}

\section{(D) Contribution of parts and components to growth of imports}

\begin{tabular}{|c|c|c|c|c|c|c|c|c|c|c|c|c|c|c|c|c|c|c|}
\hline & $\mathrm{CHN}$ & HKG & IDN & KOR & MAL & PHL & SGP & THA & TWN & VNM & JPN & ASEAN & EAS & EAS-J & NAF & USA & EUU & WLD \\
\hline CHN & --- & 15.4 & 23.7 & 29.1 & 63.2 & 80.8 & 43.5 & 46.2 & 33.5 & 12.1 & 44.7 & 51.5 & 43.6 & 42.9 & 36.1 & 31.9 & 41.3 & 41.9 \\
\hline HKG & 20.6 & --- & 12.6 & 57.6 & 56.3 & 68.9 & 81.1 & 46.3 & 48.2 & 11.9 & 132.4 & 63.8 & 38.8 & 33.1 & 56.8 & 56.1 & 24.4 & 36.7 \\
\hline IDN & 14.0 & 2.0 & --- & 45.6 & 7.8 & 43.6 & 5.9 & 11.4 & -207.7 & 50.0 & -46.2 & 9.2 & 20.3 & 4.9 & 28.6 & 33.9 & 26.7 & 74.5 \\
\hline KOR & 32.4 & 40.5 & 29.9 & --- & 74.5 & 79.4 & 18.9 & 53.1 & 75.2 & 14.5 & 45.9 & 56.8 & 48.9 & 50.7 & 62.1 & 63.7 & 50.3 & 52.1 \\
\hline MAL & 49.1 & 73.8 & 53.8 & 73.3 & --- & 93.9 & 74.3 & 62.1 & 90.7 & 62.4 & 78.1 & 72.7 & 73.8 & 72.3 & 85.4 & 86.4 & 87.9 & 77.5 \\
\hline PHL & 22.5 & 34.8 & 9.4 & 60.1 & 61.9 & --- & 65.4 & 47.1 & 50.0 & 14.3 & 89.1 & 53.3 & 59.6 & 49.2 & 91.0 & 91.4 & 59.9 & 64.4 \\
\hline SGP & 49.5 & 45.0 & 58.0 & 75.4 & 71.0 & 84.4 & \begin{tabular}{l|}
-- \\
-
\end{tabular} & 58.9 & 95.5 & 19.2 & 70.6 & 69.6 & 69.2 & 68.8 & 78.3 & 78.2 & 72.2 & 70.9 \\
\hline THA & 40.9 & 49.9 & 30.0 & 57.9 & 56.8 & 88.7 & 27.0 & $-{ }_{-1}$ & 45.0 & 90.0 & 73.5 & 53.2 & 56.1 & 49.7 & 89.0 & 86.8 & 343.9 & 62.1 \\
\hline TWN & 29.4 & 42.0 & 20.8 & 67.1 & 64.0 & 72.1 & 66.1 & 70.4 & --- & --- & 25.1 & 62.3 & 46.4 & 59.8 & 49.1 & 49.0 & 29.6 & 42.6 \\
\hline VNM & 6.8 & 25.0 & 17.8 & 16.4 & 29.9 & 14.5 & 33.4 & 23.8 & --- & --- & 32.5 & 27.8 & 22.1 & 19.2 & 37.4 & 40.6 & 18.6 & 22.1 \\
\hline JPN & 16.5 & -78.6 & 27.1 & 60.0 & 35.4 & 55.0 & 46.7 & 24.6 & 54.3 & 11.7 & --- & 37.0 & 32.2 & 32.2 & 40.3 & 41.4 & 18.9 & 32.2 \\
\hline
\end{tabular}


Table A-4: East Asia: Bilateral Trade Balances (\%)

(A) Total manufacturing trade

\begin{tabular}{|c|c|c|c|c|c|c|c|c|c|c|c|c|c|c|c|c|c|c|c|}
\hline \multirow[t]{2}{*}{ Trader } & \multicolumn{19}{|c|}{ Partner } \\
\hline & & $\mathrm{CHN}$ & $\mathrm{HKG}$ & IDN & KOR & MAL & PHL & SGP & THA & TWN & VNM & JPN & ASEAN & EAS & EAS-J & NAF & USA & $\mathrm{EU}$ & WLD \\
\hline \multirow[t]{3}{*}{$\mathrm{CHN}$} & 1992 & --- & 86.9 & -61.5 & -45.1 & 47.5 & 137.5 & 152.1 & 312.9 & -92.6 & 1733.3 & -46.5 & 48.8 & 15.5 & 41.2 & 5.4 & 6.8 & -34.1 & 8.7 \\
\hline & 1996 & --- & 295.3 & 20.2 & -58.8 & -18.4 & 454.4 & 47.9 & -1.4 & -86.5 & 5345.5 & -12.1 & 38.3 & 3.5 & 14.1 & 95.3 & 100.4 & -3.4 & 20.8 \\
\hline & 2000 & --- & 366.1 & 13.7 & -59.8 & -50.4 & -12.9 & 16.8 & -41.7 & -82.7 & 1481.2 & -10.6 & -10.1 & -2.0 & 3.0 & 166.7 & 180.3 & 27.8 & 34.5 \\
\hline \multirow[t]{3}{*}{$\mathrm{HKG}$} & 1992 & -46.5 & --- & -68.8 & -84.7 & -39.5 & --- & -36.6 & -58.4 & --- & --- & -90.9 & -44.1 & -62.1 & -50.3 & 85.0 & 69.6 & 118.8 & -45.9 \\
\hline & 1996 & -89.5 & --- & -85.6 & -96.5 & -91.9 & -61.2 & -83.7 & -86.3 & -94.4 & -6.3 & -94.9 & -84.8 & -90.9 & -90.1 & -44.5 & -45.2 & -74.6 & -85.0 \\
\hline & 2000 & -92.5 & --- & -92.6 & -97.9 & -92.5 & -85.7 & -92.3 & -94.5 & -94.9 & -74.6 & -97.4 & -91.9 & -93.7 & -93.0 & -44.5 & -43.8 & -74.0 & -88.3 \\
\hline \multirow[t]{3}{*}{ IDN } & 1992 & 45.4 & 220.7 & --- & -68.0 & 10.4 & 248.3 & 169.4 & 38.9 & -56.7 & --- & -61.1 & 146.9 & -26.8 & 12.7 & -5.0 & -4.8 & -34.4 & -18.2 \\
\hline & 1996 & -37.1 & 507.1 & --- & -62.1 & 32.9 & 339.1 & 109.8 & -13.3 & -55.4 & 2608.3 & -46.1 & 89.7 & -17.9 & 12.8 & 25.8 & 31.4 & -40.7 & -10.9 \\
\hline & 2000 & 5.0 & 323.9 & --- & -48.0 & 136.5 & 463.1 & 168.0 & 1.6 & -20.5 & 2183.3 & -0.3 & 144.5 & 39.0 & 65.6 & 187.5 & 192.3 & 91.9 & 83.9 \\
\hline \multirow[t]{3}{*}{ KOR } & 1992 & -3.0 & 554.0 & 175.8 & --- & 186.8 & 706.8 & 95.2 & 407.0 & 55.1 & 1958.8 & -53.9 & 178.7 & 2.1 & 154.8 & 47.6 & 34.8 & 4.0 & 36.9 \\
\hline & 1996 & 88.1 & 724.9 & 248.1 & --- & 145.2 & 442.8 & 113.6 & 285.4 & 20.8 & 877.8 & -62.9 & 202.8 & 13.1 & 169.1 & -10.0 & -14.6 & -17.0 & 21.4 \\
\hline & 2000 & 73.0 & 648.1 & 222.4 & --- & -1.6 & 91.5 & 55.3 & 52.6 & 63.2 & 712.1 & -50.5 & 69.2 & 18.5 & 97.3 & 60.3 & 49.3 & 70.8 & 59.2 \\
\hline \multirow[t]{3}{*}{ MAL } & 1992 & -46.4 & 65.4 & -43.9 & -75.3 & --- & -8.3 & 46.3 & -10.3 & -67.7 & 675.0 & -78.3 & 34.3 & -36.5 & 1.8 & 23.0 & 19.7 & 0.0 & -17.0 \\
\hline & 1996 & -26.6 & 141.0 & -8.7 & -72.4 & --- & -14.6 & 66.0 & 1.6 & -24.4 & 529.4 & -61.2 & 46.8 & -15.8 & 21.1 & 26.8 & 25.6 & -9.6 & -3.8 \\
\hline & 2000 & -21.0 & 87.1 & -36.1 & -43.2 & --- & -34.0 & 70.4 & 3.2 & -30.8 & 159.7 & -41.9 & 36.5 & -4.8 & 16.3 & 55.4 & 49.5 & 50.1 & 18.6 \\
\hline \multirow[t]{3}{*}{ PHL } & 1992 & -57.9 & --- & -71.3 & -87.6 & 9.1 & --- & -58.0 & -24.6 & --- & --- & -75.4 & -43.1 & -69.7 & -59.4 & 103.1 & 99.0 & 0.0 & -19.7 \\
\hline & 1996 & -86.1 & -48.0 & -75.3 & -88.6 & 12.9 & --- & -25.6 & 76.6 & -61.4 & 208.8 & -61.8 & -5.3 & -53.5 & -45.8 & 10.0 & 10.5 & -14.4 & -33.1 \\
\hline & 2000 & -30.2 & 39.2 & -71.7 & -55.8 & 45.0 & --- & 56.3 & 40.7 & 44.0 & 96.4 & -20.5 & 36.2 & 1.2 & 14.4 & 134.8 & 128.0 & 130.5 & 40.9 \\
\hline \multirow[t]{3}{*}{ SGP } & 1992 & -45.5 & 57.8 & -62.9 & -53.9 & -26.1 & 138.2 & --- & 14.8 & -12.1 & 3760.0 & -80.7 & -20.1 & -38.3 & -5.7 & 21.2 & 19.5 & 13.5 & -9.6 \\
\hline & 1996 & -44.8 & 92.6 & --- & -26.0 & 13.7 & 52.7 & --- & -8.0 & -15.4 & 711.1 & -63.8 & 13.4 & -13.6 & 13.5 & 13.7 & 14.0 & -7.3 & -2.4 \\
\hline & 2000 & -25.9 & 133.4 & -62.7 & 7.4 & 5.3 & -16.5 & --- & 13.1 & 33.2 & 605.4 & -59.3 & -2.8 & -7.5 & 16.0 & 24.5 & 22.6 & 30.6 & 8.4 \\
\hline \multirow[t]{3}{*}{ THA } & 1992 & -90.2 & 140.2 & -23.8 & -84.7 & -11.0 & 32.7 & 31.0 & --- & -76.9 & 1133.3 & -69.6 & 20.0 & -51.3 & -23.7 & 50.9 & 52.5 & -12.3 & -21.8 \\
\hline & 1996 & -41.1 & 178.9 & -14.6 & -74.2 & -18.1 & -10.3 & 115.2 & --- & -100.0 & -100.0 & -66.6 & 48.6 & -40.4 & -3.9 & 25.8 & -0.3 & -18.5 & -25.9 \\
\hline & 2000 & -43.8 & 217.8 & 13.7 & -54.8 & -17.4 & -10.8 & 42.1 & --- & -21.7 & 149.6 & -46.8 & 15.5 & -18.4 & 5.7 & 91.4 & 85.8 & 68.7 & 16.1 \\
\hline
\end{tabular}




\begin{tabular}{|c|c|c|c|c|c|c|c|c|c|c|c|c|c|c|c|c|c|c|c|}
\hline TWN & 1992 & 1251.3 & --- & 131.2 & -35.5 & 210.1 & --- & 13.8 & 332.4 & --- & --- & -69.2 & 96.9 & -18.3 & 137.2 & 150.8 & 139.2 & 253.3 & 40.7 \\
\hline & 1996 & 643.2 & 1692.8 & 124.1 & -17.2 & 32.3 & 159.1 & 18.2 & -.- & --- & -.- & -52.8 & 76.4 & 55.5 & 233.1 & 158.3 & 77.7 & 77.7 & 90.1 \\
\hline & 2000 & 479.4 & 1852.7 & 25.7 & -38.7 & 44.6 & -30.6 & -24.9 & 27.8 & --- & --- & -51.4 & -1.9 & 20.6 & 103.2 & 130.9 & 109.3 & 119.4 & 60.9 \\
\hline \multirow[t]{3}{*}{ VNM } & 1992 & -94.5 & --- & -100.0 & -95.1 & -87.1 & -- & -97.4 & -91.9 & -- & --- & -70.5 & -97.3 & -88.0 & -96.1 & -62.5 & -100.0 & 45.0 & -84.9 \\
\hline & 1996 & -98.2 & 6.7 & -96.3 & -89.8 & -84.1 & -67.6 & -87.7 & -- & --- & -- & -19.0 & -86.0 & -73.2 & -87.9 & -77.0 & 11.8 & 11.8 & -54.9 \\
\hline & 2000 & -93.7 & 293.8 & -95.6 & -87.7 & -61.5 & -49.1 & -85.8 & -59.9 & --- & --- & -6.2 & -74.3 & -63.2 & -80.9 & 28.5 & -7.1 & 273.7 & -14.3 \\
\hline \multirow[t]{3}{*}{ JPN } & 1992 & -4.7 & 996.0 & 129.4 & 101.2 & 289.5 & 306.4 & 556.9 & 189.3 & 225.2 & 238.8 & --- & 272.3 & 182.6 & 182.6 & 233.0 & 211.7 & 140.7 & 215.3 \\
\hline & 1996 & -39.2 & 943.0 & 85.0 & 141.4 & 107.1 & 147.8 & 234.5 & 163.3 & 111.8 & 23.4 & --- & 145.3 & 91.7 & 91.7 & 114.2 & 108.6 & 45.5 & 102.1 \\
\hline & 2000 & -41.4 & 1670.8 & 39.8 & 91.2 & 24.6 & 64.4 & 249.9 & 63.9 & 105.6 & 6.6 & --- & 75.6 & 54.6 & 54.6 & 174.5 & 168.6 & 92.7 & 108.0 \\
\hline
\end{tabular}

\section{(B) Trade in Parts and components}

\begin{tabular}{|c|c|c|c|c|c|c|c|c|c|c|c|c|c|c|c|c|c|c|c|}
\hline & & $\mathrm{CHN}$ & $\mathrm{HKG}$ & IDN & KOR & MAL & PHL & SGP & THA & TWN & VNM & JPN & ASEAN & EAS & EAS-J & NAF & USA & $\mathrm{EU}$ & WLD \\
\hline \multirow[t]{3}{*}{$\mathrm{CHN}$} & 1992 & --- & -64.2 & 96.0 & -215.6 & 54.5 & 90.9 & 7.6 & 52.6 & -1154.4 & 66.7 & -514.2 & 41.2 & -134.3 & -88.6 & -248.9 & -246.2 & -1004.4 & -168.5 \\
\hline & 1996 & --- & 37.3 & 88.5 & -117.4 & -25.5 & 37.3 & -45.8 & -156.1 & -398.4 & 93.8 & -184.0 & -22.8 & -80.2 & -32.8 & -24.8 & -20.3 & -204.8 & -78.5 \\
\hline & 2000 & --- & 64.6 & 13.0 & -215.3 & -305.2 & -168.5 & 4.0 & -127.3 & -507.5 & 87.7 & -171.6 & -81.3 & -73.8 & -41.3 & -13.0 & -0.6 & -146.9 & -71.4 \\
\hline \multirow[t]{3}{*}{ HKG } & 1992 & 39.1 & --- & 0.0 & -63.9 & -160.6 & --- & -5.0 & -52.2 & --- & --- & -898.6 & -37.4 & -35.3 & 13.5 & -16.2 & -25.7 & 2.6 & -29.8 \\
\hline & 1996 & -166.1 & --- & -124.2 & -1743.9 & -1178.3 & -303.7 & -239.6 & -267.6 & -1486.1 & -80.0 & -2433.4 & -326.3 & -492.0 & -341.1 & -261.4 & -274.3 & -306.6 & -408.9 \\
\hline & 2000 & -504.3 & --- & -870.0 & -3891.8 & -1181.3 & -1126.7 & -998.1 & -2162.5 & -2140.1 & -87.5 & -3702.2 & -1109.8 & -1073.8 & -863.5 & -465.3 & -445.3 & -373.2 & -837.1 \\
\hline \multirow[t]{3}{*}{ IDN } & 1992 & --- & 0.0 & --- & -2557.1 & -308.3 & -50.0 & -2.9 & 30.8 & -828.6 & --- & -1207.6 & -16.6 & -454.8 & -158.8 & -624.5 & -601.1 & -1026.4 & -524.9 \\
\hline & 1996 & -1816.7 & 64.5 & --- & -551.3 & 24.1 & 30.8 & 62.3 & -64.2 & -720.7 & -33.3 & -842.9 & 47.1 & -188.0 & -6.3 & -300.9 & \begin{tabular}{|l|}
-299.0 \\
\end{tabular} & \begin{tabular}{|l|} 
\\
\end{tabular} & -259.3 \\
\hline & 2000 & -93.2 & 80.6 & --- & -32.1 & 83.5 & 68.6 & 85.6 & 39.5 & -33.0 & 62.5 & -70.7 & 81.8 & 33.2 & 70.1 & 21.6 & 30.8 & -18.9 & 26.1 \\
\hline \multirow[t]{3}{*}{ KOR } & 1992 & 32.6 & 39.0 & 94.0 & --- & 70.4 & 63.1 & 45.5 & 70.0 & 28.8 & 92.3 & -279.9 & 55.8 & -30.7 & 48.5 & 5.3 & -1.1 & -7.4 & -9.5 \\
\hline & 1996 & 23.2 & 70.1 & 91.4 & --- & 74.6 & 44.7 & 68.0 & 59.2 & -11.0 & 90.3 & -158.6 & 70.5 & 5.3 & 57.2 & 10.4 & 5.2 & 3.9 & 10.5 \\
\hline & 2000 & 41.7 & 74.9 & 84.7 & --- & -9.0 & 28.5 & 59.1 & 21.5 & 32.0 & 87.4 & -89.7 & 35.8 & 13.6 & 42.1 & 10.2 & 2.4 & 41.7 & 20.3 \\
\hline \multirow[t]{3}{*}{ MAL } & 1992 & -225.0 & 61.6 & 76.1 & -133.3 & --- & -190.0 & 21.3 & -15.4 & -49.3 & 100.0 & -281.6 & 15.1 & -37.2 & 10.3 & 16.9 & 15.3 & 14.9 & -13.0 \\
\hline & 1996 & -106.2 & 56.2 & -13.5 & -609.8 & --- & -151.3 & 36.8 & 4.0 & 25.3 & 59.1 & -232.7 & 26.8 & -22.7 & 14.1 & -5.4 & -7.1 & -3.7 & -16.0 \\
\hline & 2000 & -4.7 & 41.0 & -153.9 & -83.5 & --- & -116.8 & 45.7 & 12.0 & -36.4 & 16.1 & -128.2 & 27.3 & -7.4 & 15.2 & 9.8 & 5.6 & 20.6 & 0.4 \\
\hline
\end{tabular}




\begin{tabular}{|c|c|c|c|c|c|c|c|c|c|c|c|c|c|c|c|c|c|c|c|}
\hline PHL & 1992 & -1000.0 & --- & 33.3 & -171.1 & 65.5 & --- & 8.5 & -96.2 & --- & --- & -381.6 & 28.3 & -123.7 & 6.9 & 11.8 & 8.3 & -7.3 & -38.4 \\
\hline & 1996 & -591.7 & -24.8 & -25.0 & -288.5 & 52.8 & -- & 23.8 & 81.8 & 11.2 & -800.0 & -179.8 & 47.8 & -41.2 & 18.2 & -15.9 & -15.9 & 20.4 & -24.0 \\
\hline & 2000 & 66.1 & 68.4 & 47.8 & -41.8 & 58.5 & --- & 60.9 & 64.8 & 51.8 & 50.0 & -42.0 & 60.9 & 32.0 & 52.2 & 40.8 & 37.8 & 63.8 & 39.0 \\
\hline \multirow[t]{3}{*}{ SGP } & 1992 & 12.2 & 4.8 & 2.8 & -188.0 & -24.9 & -9.3 & --- & -18.6 & 5.9 & 100.0 & -367.5 & -20.5 & -68.6 & -18.5 & 9.3 & 9.2 & 4.1 & -25.2 \\
\hline & 1996 & -22.2 & 36.8 & --- & -105.4 & 14.5 & -27.2 & --- & -5.4 & -7.9 & 78.6 & -220.1 & 9.1 & -24.2 & 4.7 & -6.3 & -6.1 & 10.4 & -10.3 \\
\hline & 2000 & -13.5 & 64.1 & -592.2 & -11.0 & 12.7 & -110.0 & --. & -1.6 & 13.8 & 90.6 & -183.4 & -4.8 & -9.0 & 11.3 & 11.8 & 9.9 & 34.5 & 3.8 \\
\hline \multirow[t]{3}{*}{ THA } & 1992 & -1050.0 & 34.3 & 16.7 & -338.5 & -21.7 & 49.0 & 33.8 & --- & -325.2 & 100.0 & -264.8 & 25.0 & -73.1 & -1.9 & -21.1 & -18.1 & -71.2 & -60.3 \\
\hline & 1996 & -20.7 & 36.3 & 33.3 & -186.5 & -22.7 & -212.4 & 48.7 & --. & --- & --. & -440.8 & 20.6 & -95.9 & -4.4 & -67.3 & \begin{tabular}{|l|}
-115.9 \\
\end{tabular} & -57.8 & -89.5 \\
\hline & 2000 & -11.3 & 66.4 & -3.3 & -24.0 & -21.3 & -87.2 & 57.5 & --- & 36.1 & -57.3 & -100.1 & 19.9 & -6.9 & 22.0 & -3.5 & -9.9 & 55.6 & 4.4 \\
\hline \multirow[t]{3}{*}{ TWN } & 1992 & 92.0 & --- & 89.2 & -40.5 & 33.0 & --- & -6.3 & 76.5 & --- & - & -472.7 & 31.4 & -81.6 & 41.8 & 46.0 & 43.0 & 69.0 & -4.4 \\
\hline & 1996 & 79.9 & 93.7 & 87.8 & 9.9 & -33.9 & -12.6 & 7.3 & 100.0 & -.- & --- & -197.2 & 14.1 & 7.1 & 53.8 & 61.2 & 52.8 & 52.8 & 36.0 \\
\hline & 2000 & 83.5 & 95.5 & 24.8 & -47.0 & 26.7 & -107.6 & -16.1 & -56.5 & --. & -.- & -48.1 & -17.5 & 20.6 & 38.7 & 53.1 & 47.4 & 62.2 & 38.7 \\
\hline \multirow[t]{3}{*}{ VNM } & 1992 & -200.0 & --- & --- & -1200.0 & --- & --- & --- & --- & --- & --- & -900.0 & -- & -975.0 & -1000.0 & --- & --- & -- & -1275.0 \\
\hline & 1996 & -1500.0 & 44.4 & 25.0 & -926.7 & -144.4 & 88.9 & -367.6 & 100.0 & -- & --. & -221.7 & -114.1 & -228.7 & -229.9 & --. & -2175.0 & -2175.0 & -371.7 \\
\hline & 2000 & -711.1 & 46.7 & -166.7 & -692.0 & -19.2 & -100.0 & -962.5 & 36.4 & --- & --- & -142.7 & -58.2 & -121.4 & -112.8 & -3333.3 & $-5050.0 \mid$ & -388.9 & -155.6 \\
\hline \multirow[t]{3}{*}{ JPN } & 1992 & 60.1 & 90.0 & 92.2 & 72.0 & 82.6 & 79.2 & 86.4 & 72.4 & 82.5 & 90.0 & -- & 82.3 & 80.7 & 80.7 & 72.4 & 70.4 & 81.7 & 78.7 \\
\hline & 1996 & 30.6 & 90.4 & 83.8 & 50.8 & 78.4 & 66.1 & 77.0 & 77.9 & 66.3 & 68.9 & --- & 76.7 & 69.1 & 69.1 & 60.2 & 57.8 & 75.6 & 68.4 \\
\hline & 2000 & 28.6 & 93.2 & 72.5 & 46.2 & 46.8 & 43.1 & 73.5 & 64.7 & 32.5 & \begin{tabular}{|l|}
58.8 \\
\end{tabular} & -- & 60.1 & 54.8 & 54.8 & 60.4 & 57.9 & 78.2 & 61.9 \\
\hline \multicolumn{20}{|c|}{ onents } \\
\hline & & CHN & HKG & IDN & KOR & MAL & PHL & SGP & THA & TWN & VNM & JPN & ASEAN & EAS & EAS-J & NAF & USA & $\mathrm{EU}$ & WLD \\
\hline \multirow[t]{3}{*}{ CHN } & 1992 & --- & 56.2 & -187.8 & -72.3 & 29.6 & 54.9 & 65.5 & 76.9 & -1275.2 & 96.2 & -58.4 & 32.1 & 26.1 & 39.6 & 16.1 & 17.8 & -23.0 & 20.7 \\
\hline & 1996 & --- & 79.4 & 2.4 & -147.7 & -21.5 & 89.5 & 51.7 & 18.8 & -726.8 & 98.5 & 9.0 & 38.5 & 15.8 & 19.4 & 54.4 & 55.5 & 9.3 & 27.6 \\
\hline & 2000 & --- & 82.3 & 11.9 & -130.0 & -14.6 & 61.4 & 20.5 & -42.8 & -468.2 & 94.1 & 17.1 & 17.3 & 16.6 & 16.3 & 70.3 & 70.8 & 42.4 & 42.1 \\
\hline \multirow[t]{3}{*}{ HKG } & 1992 & -128.3 & --- & -259.4 & -2297.0 & -21.5 & --- & -104.8 & -169.0 & -- & -- & -1023.1 & -105.5 & -214.1 & -147.7 & 54.4 & 50.3 & 58.8 & -99.9 \\
\hline & 1996 & -1249.0 & -- & -707.4 & -3450.9 & -1106.9 & -68.4 & -1217.1 & -827.1 & -1799.6 & 0.0 & -1634.6 & -816.5 & -1321.0 & -1276.2 & -45.0 & -45.0 & -291.5 & -624.9 \\
\hline & 2000 & -1624.5 & --. & -1304.0 & $\mid-5061.3$ & -1307.7 & -257.1 & -1648.9 & -1546.3 & -1644.3 & -362.5 & -3852.8 & -1150.4 & -1741.1 & -1613.6 & -19.4 & -19.5 & -262.9 & -728.1 \\
\hline \multirow[t]{3}{*}{ IDN } & 1992 & 36.9 & 72.2 & --- & -180.9 & 27.6 & 73.7 & 68.6 & 27.4 & -98.0 & 100.0 & -98.7 & 65.7 & -13.2 & 20.9 & 15.4 & 15.7 & -26.1 & -1.2 \\
\hline & 1996 & -39.1 & 85.0 & --- & -143.0 & 24.9 & 79.5 & 49.3 & -7.2 & -96.1 & 98.7 & -27.3 & 47.3 & -0.7 & 14.1 & 35.2 & 38.5 & -36.7 & 7.7 \\
\hline & 2000 & 10.7 & 75.8 & --- & -99.4 & 43.6 & 85.3 & 50.5 & -7.9 & -24.7 & \begin{tabular}{|l|l|}
97.7 \\
\end{tabular} & 18.2 & 48.7 & 26.5 & 30.1 & 69.6 & 69.4 & 52.7 & 48.9 \\
\hline
\end{tabular}




\begin{tabular}{|c|c|c|c|c|c|c|c|c|c|c|c|c|c|c|c|c|c|c|c|}
\hline KOR & 1992 & -6.3 & 95.8 & 60.4 & --- & 58.1 & 92.8 & 55.2 & 82.8 & 38.3 & 95.3 & -80.5 & 69.4 & 13.1 & 65.6 & 38.7 & 32.7 & 5.6 & 34.9 \\
\hline & 1996 & 50.4 & 93.7 & 67.2 & --- & 24.8 & 94.2 & 32.1 & 78.0 & 31.9 & 89.7 & -174.5 & 64.6 & 14.2 & 65.2 & -23.7 & -30.6 & -28.1 & 20.0 \\
\hline & 2000 & 42.4 & 93.1 & 64.8 & --- & 13.3 & 73.4 & -11.3 & 43.4 & 48.1 & 87.7 & -110.3 & 46.0 & 16.9 & 54.4 & 49.5 & 46.4 & 41.4 & 44.6 \\
\hline \multirow[t]{3}{*}{ MAL } & 1992 & -77.0 & 17.7 & -139.7 & -744.3 & --- & 84.5 & 40.2 & -6.4 & -398.0 & 86.2 & -427.1 & 34.1 & -74.5 & -5.3 & 20.1 & 17.4 & -7.2 & -25.5 \\
\hline & 1996 & -22.7 & 60.2 & -8.0 & -75.1 & --- & 81.9 & 44.1 & -2.3 & -137.2 & 90.6 & -116.6 & 38.6 & -15.1 & 21.1 & 38.8 & 39.3 & -14.4 & 4.9 \\
\hline & 2000 & -48.9 & 52.7 & -19.8 & -64.7 & --- & 71.7 & 32.0 & -15.4 & -57.7 & 78.9 & -33.7 & 25.7 & -1.9 & 12.2 & 58.7 & 58.1 & 43.4 & 30.9 \\
\hline \multirow[t]{3}{*}{ PHL } & 1992 & -121.8 & --- & -280.8 & -1288.6 & -544.4 & --- & -426.2 & -10.7 & --- & --- & -278.6 & -264.5 & -306.5 & -359.4 & 64.4 & 64.3 & 1.3 & -18.7 \\
\hline & 1996 & -625.0 & -179.2 & -407.3 & -1761.7 & -470.6 & --- & -303.1 & -389.8 & -495.3 & 93.1 & -145.1 & -257.6 & -247.5 & -379.1 & 27.7 & 29.0 & -64.3 & -77.4 \\
\hline & 2000 & -535.5 & -126.1 & -588.7 & -412.0 & -382.7 & --- & -116.1 & -381.5 & -24.3 & 48.9 & -7.6 & -214.6 & -81.7 & -160.6 & 75.8 & 76.0 & 42.3 & 11.4 \\
\hline \multirow[t]{3}{*}{ SGP } & 1992 & -112.1 & 51.2 & -218.7 & -84.5 & -42.1 & 81.0 & --- & 37.1 & -26.6 & 97.2 & -447.9 & -27.9 & -58.3 & 0.7 & 20.6 & 19.1 & 13.8 & -4.9 \\
\hline & 1996 & -123.8 & 55.1 & --- & 6.5 & 9.3 & 80.4 & --- & -11.1 & -28.2 & 89.5 & -149.4 & 14.4 & -9.3 & 17.5 & 23.5 & 23.8 & -19.5 & 2.7 \\
\hline & 2000 & -56.8 & 48.6 & -102.0 & 24.5 & -8.3 & 61.3 & --- & 25.6 & 40.3 & 84.0 & -119.5 & -0.4 & -7.1 & 17.0 & 26.7 & 25.9 & 13.3 & 11.6 \\
\hline \multirow[t]{3}{*}{ THA } & 1992 & -912.7 & 62.8 & -38.3 & -644.8 & -0.8 & 9.6 & 7.5 & --- & -334.7 & 91.4 & -217.1 & 5.6 & -123.6 & -52.0 & 44.7 & 45.4 & -6.5 & -19.1 \\
\hline & 1996 & -90.0 & 70.1 & -29.9 & -338.5 & -21.1 & 72.7 & 56.8 & --- & --- & --- & -138.6 & 41.5 & -54.9 & -3.8 & 37.8 & 22.5 & -15.8 & -18.2 \\
\hline & 2000 & -156.0 & 69.6 & 16.5 & -274.2 & -20.8 & 61.4 & -32.9 & --- & -127.2 & 94.2 & -80.9 & 4.8 & -36.6 & -12.3 & 66.6 & 66.2 & 33.6 & 19.2 \\
\hline \multirow[t]{3}{*}{ TWN } & 1992 & 92.7 & --- & 49.5 & -62.1 & 79.9 & --- & 21.0 & 77.0 & --- & --- & -166.6 & 55.7 & -5.1 & 62.9 & 63.4 & 61.7 & 72.4 & 37.3 \\
\hline & 1996 & 87.9 & 94.7 & 49.0 & -46.9 & 57.8 & 83.2 & 22.0 & 100.0 & --- & --- & -79.3 & 58.5 & 46.8 & 76.3 & 61.3 & 40.3 & 40.3 & 52.0 \\
\hline & 2000 & 82.4 & 94.3 & 19.8 & -92.5 & 36.6 & 19.6 & -67.4 & 56.0 & --- & --- & -146.5 & 12.9 & 14.4 & 60.1 & 58.5 & 54.6 & 50.3 & 37.4 \\
\hline \multirow[t]{3}{*}{ VNM } & 1992 & -2500.0 & --- & --- & -2006.3 & -625.0 & --- & -3500.0 & -1066.7 & --- & --- & -233.0 & -3466.7 & -727.6 & -2623.3 & -133.3 & --- & 55.2 & -547.7 \\
\hline & 1996 & -6787.5 & 0.0 & -7875.0 & -872.1 & -962.5 & -1357.1 & -851.8 & 100.0 & --- & --- & -17.6 & -1013.7 & -279.0 & -928.6 & -290.9 & 21.5 & 21.5 & -108.1 \\
\hline & 2000 & -1596.7 & 78.4 & -4200.0 & -715.3 & -374.5 & -95.8 & -524.5 & -1623.1 & --- & --- & 9.8 & -589.4 & -186.1 & -635.3 & 50.3 & 35.3 & 78.1 & -3.4 \\
\hline \multirow[t]{3}{*}{ JPN } & 1992 & -13.6 & 91.1 & 45.1 & 40.4 & 68.4 & 73.6 & 83.9 & 62.4 & 62.5 & 70.0 & --- & 68.6 & 58.3 & 58.3 & 69.1 & 67.1 & 52.9 & 65.2 \\
\hline & 1996 & -93.9 & 90.4 & 29.4 & 62.0 & 25.3 & 53.9 & 64.9 & 53.7 & 44.2 & 15.0 & --- & 47.3 & 36.4 & 36.4 & 50.0 & 49.4 & 15.3 & 42.8 \\
\hline & 2000 & -119.0 & 95.1 & -7.0 & 48.5 & -9.0 & 34.4 & 69.7 & 20.1 & 59.4 & -10.9 & --- & 28.0 & 23.2 & 23.2 & 64.8 & 64.6 & 36.6 & 47.5 \\
\hline
\end{tabular}

\section{Notes}

1. Trade balance (exports-imports) expressed as a percentage of exports. 
Table A-5: List of Parts and Components Identified at the Five-digit Level of the Standard International Classification (SITC) System (Revision 3)*

\begin{tabular}{|c|c|}
\hline SITC & DESCRIPTION \\
\hline SITC 7 & Machinery and Transport Equipment \\
\hline 71191 & Parts for steam generating or other vapour generating boilers \\
\hline 71192 & Parts for auxiliary plants used with boilers; parts of condensers for steamers and other vapour power units \\
\hline 71280 & Parts for steam turbines and other vapour turbines \\
\hline 71319 & Parts, n.e.s., of spark-ignition reciprocating or rotary combustion piston engines for aircraft \\
\hline 71331 & Outboard motors \\
\hline 71332 & Spark-ignition reciprocating or rotary marine propulsion engines, n.e.s. \\
\hline 71391 & Parts, n.e.s, suitable for use solely or principally with spark-ignition internal combustion piston engines \\
\hline 71392 & Parts, n.e.s., suitable for use solely or principally with compression-ignition internal combustion piston engines \\
\hline 71491 & Parts for turbojets or turbo propellers \\
\hline 71499 & Parts for gas turbines, n.e.s. \\
\hline 71690 & Parts n.e.s. for use solely or principally with electric motors, electric generators, electric generating sets and rotary converters \\
\hline 71819 & Parts, including regulators, of hydraulic turbines and water wheels \\
\hline 71878 & Parts of nuclear reactors \\
\hline 71899 & Parts of reaction engines (except turbojet), linear acting hydraulic and pneumatic power engines and motors, and parts of engines and motors n.e.s. \\
\hline 72119 & Parts of agricultural, horticultural or forestry machinery for soil preparation or cultivation and lawn or sports ground rollers \\
\hline 72129 & Parts of harvesting or threshing machines, mowers, and machines for cleaning, sorting and grading eggs, fruit or other agricultural produce \\
\hline
\end{tabular}




\begin{tabular}{|c|c|}
\hline 72139 & Parts for milking machines and dairy machinery \\
\hline 72198 & Parts of presses, crushers and similar machinery for the manufacture of wine, cider, fruit juices, etc. \\
\hline 72199 & Parts of agricultural, horticultural, forestry and poultry-keeping or bee-keeping machinery, n.e.s. \\
\hline 72392 & Bulldozer or angledozer blades \\
\hline 72393 & Parts for boring or sinking machinery \\
\hline 72399 & Parts n.e.s., of civil engineering etc. machinery, including mining and public works machinery parts (heading 723 ) and cranes etc. (heading 744.3 ) \\
\hline 72439 & Sewing machine needles; sewing machine furniture, bases and covers and parts thereof; sewing machine parts \\
\hline 72449 & Parts and accessories of textile machinery designed for use in the preparation and production of textile fibers and yarns \\
\hline 72467 & Parts and accessories of weaving machines (looms) or their auxiliary machines \\
\hline 72468 & Parts and accessories of knitting and stitch-bonding machines, tulle, lace, embroidery, net, etc. machines or their auxiliary machines \\
\hline 72488 & Parts for leather machinery designed for preparing, tanning or working hides or leather or for making or repairing footwear or other leather articles \\
\hline 72491 & Parts for household or laundry type washing machines \\
\hline 72492 & Parts for textile machinery designed for washing, drying, bleaching, dyeing, etc. yarn, fabric or articles (not for household or laundry type washers) \\
\hline 72591 & Parts of machines for making pulp of fibrous celluloid material or for making or finishing paper or paperboard \\
\hline 72599 & Parts of machinery for making up paper pulp, paper or paperboard, n.e.s. \\
\hline 72689 & Parts for bookbinding machinery \\
\hline 72691 & Parts for typesetting and type-founding machinery, apparatus and equipment \\
\hline 72699 & Parts for printing machinery and parts of machines for uses ancillary to printing \\
\hline 72719 & Parts for machinery (other than farm type) used for grain milling or the working, cleaning, sorting etc. of cereals or dried leguminous vegetables \\
\hline 72729 & Parts for the machinery, n.e.s. for the industrial preparation or manufacture of food or drink \\
\hline 72819 & Parts and accessories suitable for use solely or principally with machine tools specialized for particular industries \\
\hline 72839 & Parts of machinery for sorting, washing, crushing or mixing earth, stone, ores etc., and for shaping solid mineral fuels, ceramic pastes etc. \\
\hline
\end{tabular}




\begin{tabular}{|c|c|}
\hline 72851 & Parts for machines assembling electric or electronic lamps, tubes, etc.; parts for machines manufacturing or hot working glass or glassware \\
\hline 72852 & Parts of machinery for working rubber or plastics or manufacturing products made from rubber or plastics, n.e.s. \\
\hline 72853 & Parts of machinery for preparing or making up tobacco, n.e.s. \\
\hline 72855 & Parts, n.e.s., of machinery for public works etc., preparing animal or fixed vegetable fats and oils, and specialized for particular industries n.e.s. \\
\hline 73591 & Parts, n.e.s., and accessories suitable solely or principally for use with metalworking machine tools working by removing metal or other material \\
\hline 73595 & Parts, n.e.s., and accessories suitable solely or principally for use with metalworking machine tools working without removing metal or other material \\
\hline 73719 & Parts of metalworking converters, ladles, ingot moulds and casting machines \\
\hline 73729 & Rolls and other parts for metal-rolling mills \\
\hline 73739 & Parts for electric laser, other light or photon beam, ultrasonic etc. soldering, brazing or welding machines and apparatus for hot metal etc. spraying \\
\hline 73749 & Parts for machinery and apparatus for soldering, brazing or welding, n.e.s. and parts for gas-operated surface tempering machines \\
\hline 74128 & Parts for furnace burners, mechanical stokers, grates, ash dischargers and similar mechanical appliances for furnaces \\
\hline 74135 & Parts for electric industrial or laboratory furnaces and ovens, etc. \\
\hline 74139 & Parts for nonelectrical industrial or laboratory furnaces and ovens \\
\hline 74149 & Parts of refrigerators, freezers and other refrigerating or freezing equipment (electric or other) \\
\hline 74159 & Parts for the air conditioning machines (having a motor-driven fan and elements for changing the temperature and humidity) of heading 741.5 \\
\hline 74172 & Parts for producer gas or water gas generators; parts for acetylene gas generators and similar water process gas generators \\
\hline 74190 & Parts, n.e.s. for temperature changing industrial and laboratory machinery and equipment \\
\hline 74291 & Parts of pumps for liquids \\
\hline 74295 & Parts of liquid elevators \\
\hline 74380 & Parts for air or vacuum pumps, air or other gas compressors and fans; parts of ventilating, recycling or cooker hoods incorporating a fan \\
\hline 74391 & Parts of centrifuges (including centrifugal dryers) \\
\hline 74395 & Parts of filtering or purifying machinery and apparatus \\
\hline
\end{tabular}




\begin{tabular}{|c|c|}
\hline 74419 & Parts of self-propelled works trucks (electrically operated or not), not fitted with lifting etc. equipment and railway station platform tractors \\
\hline 74491 & Parts of pulley tackle, hoists, winches, capstans and jacks \\
\hline 74492 & Parts of lifting and handling machinery \\
\hline 74493 & Parts of lifts, skip hoists or escalators \\
\hline 74494 & Parts of lifting, handling, loading or unloading machinery, n.e.s. \\
\hline 74519 & Parts of tools for working in the hand, pneumatic or with self-contained nonelectric motor \\
\hline 74529 & Parts of dish washing machines (including household type), machinery for cleaning, filling, sealing, labelling, etc. containers and aerating beverages \\
\hline 74539 & Weighing machine weights; parts of weighing machinery (excluding balances of a sensitivity of 5 ca or better), weight-operated counting machines, etc. \\
\hline 74568 & Parts of mechanical appliances for projecting, dispersing or spraying liquids or powders, fire extinguishers, steam or sand blasting machines, etc. \\
\hline 74591 & Calendaring or other rolling machines (other than for metal or glass) \\
\hline 74593 & Parts (cylinders etc.) for calendaring or other rolling machines (other than for metals or glass) \\
\hline 74595 & Automatic goods-vending machines (postage stamp, cigarette, food, beverage etc.), including money-changing machines \\
\hline 74597 & Parts for automatic goods-vending machines (postage stamp, cigarette, food etc.) \\
\hline 74699 & Parts of ball and roller bearings, n.e.s. \\
\hline 74790 & Parts for taps, cocks, valves and similar appliances for pipes, boiler shells, tanks, etc. \\
\hline 74839 & Parts of articulated link chain \\
\hline 74890 & Parts, n.e.s., for transmission shafts and cranks, bearing housings, gears, gearing, ball screws, gear boxes, flywheels and pulleys, clutches, etc. \\
\hline 74991 & Ships' or boats' propellers and blades therefore \\
\hline 74999 & Machinery parts, not containing electrical connectors, insulators, coils, contacts or other electrical features, n.e.s. \\
\hline 75910 & Parts and accessories of photocopying and thermo-copying apparatus \\
\hline 75991 & Parts of typewriters and word processing machines \\
\hline 75993 & Parts of office machines, n.e.s. \\
\hline
\end{tabular}




\begin{tabular}{|c|c|}
\hline 75995 & Parts of calculating machines, accounting machines, cash registers. postage-franking machines and similar machines incorporating a calculating device \\
\hline 75997 & Parts of automatic data processing machines and units thereof, magnetic or optical readers, and machines for transcribing and processing data n.e.s. \\
\hline 75999 & Parts and accessories equally suitable for use with two or more of the machines of $7511,7519,752$, or the electronic calculating machines of 7512 \\
\hline 76491 & Parts of electrical apparatus for line telephony or line telegraphy (including apparatus for carrier-current line systems) \\
\hline 76492 & Parts of microphones, loudspeakers, headphones, earphones and combined microphone/speaker sets; audio-frequency electric amplifiers; etc. \\
\hline 76493 & Parts of television receivers, radiobroadcast receivers, transmission apparatus for radio telephony, telegraphy, broadcasting or television etc. \\
\hline 76499 & Parts of the apparatus for sound recorders or reproducers and parts of television image and sound recorders or reproducers \\
\hline 77129 & Parts of electric power machinery (other than rotating electric power generating machinery and equipment), and parts thereof \\
\hline 77220 & Printed circuits \\
\hline 77231 & Fixed carbon electrical resistors, composition or film types \\
\hline 77232 & Fixed electrical resistors, n.e.s \\
\hline 77233 & Wirewound electrical variable resistors (including rheostats and potentiometers) \\
\hline 77235 & Variable electrical resistors (including reostats and potentiometers), n.e.s. \\
\hline 77238 & Parts for the electrical resistors (including rheostats and potentiometers), other than heating resistors; and parts thereof \\
\hline 77241 & Fuses for electrical apparatus used with circuits exceeding 1,000 volts \\
\hline 77242 & Automatic circuit breakers for a voltage of less than $72.5 \mathrm{kv}$ \\
\hline 77243 & Automatic circuit breakers for a voltage of $72.5 \mathrm{kv}$ or greater \\
\hline 77244 & Electrical isolating switches and make-and-break switches for a voltage exceeding 1,000 volts \\
\hline 77245 & Lightning arresters, voltage limiters and surge suppressors for a voltage exceeding 1,000 volts \\
\hline 77249 & Electrical apparatus for switching or protecting electrical circuits, or making connections to or in electrical circuits n.e.s., exceeding 1,000 volts \\
\hline 77251 & Fuses for electrical apparatus used with circuits not exceeding 1,000 volts \\
\hline
\end{tabular}




\begin{tabular}{|c|c|}
\hline 77252 & Automatic circuit breakers for a voltage not exceeding 1,000 volts \\
\hline 77253 & Apparatus for protecting electrical circuits, n.e.s., not exceeding 1,000 volts \\
\hline 77254 & Relays for electrical apparatus used with electrical circuits not exceeding 1,000 volts \\
\hline 77255 & Switches for electrical apparatus, n.e.s., for voltages not exceeding 1,000 volts \\
\hline 77257 & Electric lamp holders, for voltages not exceeding 1,000 volts \\
\hline 77258 & Electric plugs and sockets, for voltages not exceeding 1,000 volts \\
\hline 77259 & Electrical apparatus for switching or protecting electrical circuits or making connections to or in electrical circuits, n.e.s., not exceeding $1,000 \mathrm{v}$ \\
\hline 77261 & Boards, panels, consoles and other bases, for electric control or distribution of electricity, for a voltage not exceeding 1,000 volts \\
\hline 77262 & Boards, panels, consoles and other bases, for electric control or distribution of electricity, for a voltage exceeding 1,000 volts \\
\hline 77281 & Boards, panels, consoles, desk, cabinets and other bases not equipped with their electrical apparatus \\
\hline 77282 & Parts of electrical apparatus for switching or protecting electrical circuits for making connections to or in electrical circuits, n.e.s. \\
\hline 77429 & Electro-diagnostic apparatus for medical, surgical, dental or veterinary sciences and radiological apparatus, n.e.s., including parts and accessories \\
\hline 77549 & Parts of shavers and hair clippers with self-contained electric motor (excluding blades and cutter heads) \\
\hline 77579 & Parts of electromechanical domestic appliances with self-contained electric motors \\
\hline 77589 & Parts of electrochemical appliances n.e.s. \\
\hline 77611 & Television picture tubes, colour \\
\hline 77612 & Television picture tubes, black and white or other monochrome \\
\hline 77621 & Television camera tubes; image converters and intensifiers; other photo cathode tubes \\
\hline 77623 & Cathode ray-tubes, n.e.s. \\
\hline 77625 & Microwave tubes (excluding grid-controlled tubes) \\
\hline 77627 & Electronic valves and tubes, n.e.s. \\
\hline
\end{tabular}




\begin{tabular}{|c|c|}
\hline 77629 & Parts of television picture tubes and other electronic valves and tubes \\
\hline 77631 & Diodes, not photosensitive nor light emitting diodes \\
\hline 77632 & Transistors (excluding photosensitive transistors) with a dissipation rate of less than 1 watt \\
\hline 77633 & Transistors (excluding photosensitive transistors) with a dissipation rate of 1 watt or more \\
\hline 77635 & Thyristors, diacs and triacs (excluding photosensitive devices) \\
\hline 77637 & Photosensitive semiconductor devices; light emitting diodes \\
\hline 77639 & Semiconductor devices, n.e.s. \\
\hline 77641 & Digital monolithic integrated units \\
\hline 77643 & Nondigital monolithic integrated units \\
\hline 77645 & Hybrid integrated circuits \\
\hline 77649 & Electronic integrated circuits and micro assemblies, n.e.s. \\
\hline 77681 & Piezoelectric crystals, mounted \\
\hline 77688 & Parts of diodes, transistors and similar semiconductor devices (including photosensitive), light emitting diodes and mounted piezoelectric crystals \\
\hline 77689 & Parts of electronic integrated circuits and micro assembles \\
\hline 77811 & Primary cells and primary batteries \\
\hline 77812 & Electric accumulators (storage batteries) \\
\hline 77817 & Parts of primary cells and primary batteries \\
\hline 77819 & Parts of electric accumulators \\
\hline 77829 & Parts of electric filament or discharge lamps (including parts of sealed beam lamp units and ultraviolet or infrared lamps) and arc lamps \\
\hline 77833 & Parts of electrical ignition or starting equipment for internal combustion engines; parts of generators and cut outs used with those engines \\
\hline 77835 & Parts of electrical lighting and signalling equipment (excluding filament, discharge or arc lamps); parts of windshield wipers, defrosters or demisters \\
\hline 77848 & Parts of electromechanical tools for working in the hand, with self-contained electric motor \\
\hline
\end{tabular}




\begin{tabular}{|c|c|}
\hline 77869 & Parts of electrical capacitors \\
\hline 77883 & Parts of electrical signalling, safety or traffic control equipment for railways, tramways, roads, waterways, parking facilities, etc. \\
\hline 77885 & Parts of electric sound or visual signalling apparatus, n.e.s. (including parts of indicator panels, burglar and fire alarms) \\
\hline 77889 & Electrical parts of machinery or apparatus, n.e.s. \\
\hline 78421 & Bodies (including cabs), for motor cars and motor vehicles designed for the transport of persons (other than public-transport type vehicles) \\
\hline 78425 & Bodies (including cabs) for tractors, trucks and special purpose motor vehicles and road motor vehicles n.e.s. \\
\hline 78431 & Bumpers and parts thereof, for tractors, motor cars and other motor vehicles, etc. \\
\hline 78432 & Other parts and accessories of motor vehicle bodies of headings 8701 to 8705 (including cabs) \\
\hline 78433 & Brakes and servo-brakes and parts thereof for tractors, motor cars and other motor vehicles, etc. \\
\hline 78434 & Gear boxes \\
\hline 78435 & Drive axles with differential, whether or not provided with other transmission components, for tractors, motor cars and other motor vehicles, etc. \\
\hline 78436 & Non-driving axles and parts thereof for tractors, motor cars and other motor vehicles, etc. \\
\hline 78439 & Parts and accessories n.e.s. for tractors, motor cars and other motor vehicles, trucks, public-transport vehicles and road motor vehicles, n.e.s. \\
\hline 78535 & Parts and accessories for motorcycles (including mopeds) \\
\hline 78536 & Parts and accessories for invalid carriages \\
\hline 78537 & Parts and accessories for bicycles and other cycles (except motorcycles and mopeds), n.e.s. \\
\hline 78689 & Parts of trailers and semi-trailers, for housing or camping, transport of goods, trailers, n.e.s. and vehicles not mechanically propelled, n.e.s. \\
\hline 79199 & Parts of railway or tramway locomotives or rolling stock railway vehicles; parts of railway or tramway coaches, vans, trucks, service vehicles, etc. \\
\hline 79291 & Propellers and rotors and parts thereof for aircraft \\
\hline 79293 & Undercarriages and parts thereof for aircraft \\
\hline 79295 & Parts of airplanes or helicopters, n.e.s. \\
\hline 79297 & Parts of aircraft and associated equipment, spacecraft (including satellites) and spacecraft launch vehicles, n.e.s. \\
\hline
\end{tabular}




\begin{tabular}{|c|c|c|c|}
\hline & & SITC 8 & Miscellaneous manufacturing \\
\hline & & 81211 & Radiators and parts thereof, of iron or steel \\
\hline & & 81219 & Parts of central heating boilers, n.e.s., of iron or steel \\
\hline & & 81380 & Parts of portable electric lamps designed to function on their own energy sources (batteries etc.), other than for motor vehicles, etc. \\
\hline & & 81391 & Parts n.e.s., of lamps and lighting fittings, n.e.s., and parts of illuminated signs and nameplates, etc., of glass \\
\hline & & 81392 & Parts n.e.s., of lamps and lighting fittings, n.e.s., and parts of illuminated signs and nameplates, etc., of plastics \\
\hline & & 81399 & Parts n.e.s., of lamps and lighting fittings, n.e.s., and parts of illuminated signs and nameplates, etc., of materials other than glass or plastics \\
\hline & & 82119 & Parts of seats, n.e.s. \\
\hline & & 82180 & Parts of furniture, n.e.s., of metal, wood and other materials \\
\hline & & 84699 & Clothing accessories, n.e.s., knitted or crocheted; parts of garments or of clothing accessories, knitted or crocheted \\
\hline & & 84848 & Headbands, linings, covers, hat foundations, hat frames, peaks (visors) and chinstraps, for headgear \\
\hline & & 85190 & Parts of footwear; removable in-soles, heel cushions and similar articles; gaiters, leggings and similar articles, and parts thereof \\
\hline & & 87119 & Parts and accessories (including mountings) of binoculars, monoculars, other optical telescopes, and astronomical instruments, n.e.s. \\
\hline & & 87149 & Parts and accessories of compound optical microscopes \\
\hline & & 87240 & Medical, dental, surgical or veterinary furniture (operating and examining tables, mechanical hospital beds and dentists or similar chairs) and parts \\
\hline & & 87319 & Parts and accessories of gas, liquid or electricity meters \\
\hline & & 87329 & Parts and accessories of revolution and production counters, odometers, pedometers, speedometers, tachometers, stroboscopes, etc. \\
\hline & & 87412 & Parts and accessories of navigational instruments and appliances \\
\hline & & 87414 & Parts and accessories for surveying, hydrographic, oceanographic, hydrological, meteorological, etc. instruments and appliances, and rangefinders \\
\hline & & 87424 & Parts and accessories for drafting tables, etc., marking out and mathematical calculating instruments, and instruments for measuring length by hand \\
\hline & & 87426 & Parts and accessories for measuring or checking instruments, appliances and machines, n.e.s. and profile projectors \\
\hline
\end{tabular}




\begin{tabular}{|c|c|}
\hline 87439 & Parts and accessories for instruments and apparatus for measuring or checking the flow, level, pressure or other variables of liquids or gases, n.e.s. \\
\hline 87449 & Microtones; parts and accessories of instruments for physical or chemical analysis, measuring or checking viscosity, etc. or heat, sound or light \\
\hline 87454 & Parts and accessories for the machines and appliances for testing the mechanical properties of materials \\
\hline 87456 & Parts and accessories for hydrometers and similar floating instruments, thermometers, pyrometers, pyrometers, barometers, hygrhydrometers and similar floating instruments \\
\hline 87469 & Parts and accessories for automatic regulating or controlling instruments and apparatus \\
\hline 87479 & Parts and accessories for instruments and apparatus (except meters) measuring etc. electrical quanities and devices measuring etc. ionized radiations \\
\hline 87490 & Parts and accessories for machines, appliances, instruments and apparatus, n.e.s., measuring, checking, analyzing or controlling, n.e.s. \\
\hline 88114 & Parts and accessories for photographic (other than cinematographic) cameras \\
\hline 88115 & Parts and accessories for photographic flashlight apparatus \\
\hline 88123 & Parts and accessories for cinematographic cameras \\
\hline 88124 & Parts and accessories for cinematographic projectors \\
\hline 88134 & Parts and accessories for microform readers, image projectors, n.e.s. and photographic (except cinematographic) enlargers and reducers \\
\hline 88136 & Parts and accessories for photographic (including cinematographic) laboratory apparatus and equipment, n.e.s., negascopes and projection screens \\
\hline 88422 & Parts for frames and mountings of spectacles, goggles and the like \\
\hline 88431 & Objective lenses for camera, projectors or photographic enlargers or reducers \\
\hline 88432 & Objective lenses, n.e.s., mounted \\
\hline 88433 & Filters (optical elements), mounted \\
\hline 88439 & Mounted optical elements, n.e.s. \\
\hline 88591 & Watch cases and parts of watch cases \\
\hline 88592 & Watch straps, watch bands and watch bracelets, and parts thereof, of mrtal \\
\hline 88593 & Watch straps, watch bands and watch bracelets and parts thereof, of material other than metal \\
\hline 88597 & Clock cases and cases of a similar type for other clock mechanisms (time registers and/or recorders, time stamps, time meters, etc.) and parts thereof \\
\hline
\end{tabular}




\begin{tabular}{|c|c|}
\hline 88599 & Clock or watch parts, n.e.s. \\
\hline 89124 & Cartridges and parts thereof, n.e.s. \\
\hline 89129 & Munitions of war and parts thereof, n.e.s. \\
\hline 89191 & Parts and accessories of revolvers or pistols designed to fire live ammunition \\
\hline 89195 & Parts of shotguns and rifles for sports, hunting, etc. n.e.s. \\
\hline 89199 & Parts and accessories, n.e.s. of military weapons (other than revolvers and pistols) and non-military arms (other than firearms and side arms) \\
\hline 89410 & Baby carriages, and parts thereof, n.e.s. \\
\hline 89935 & Parts and accessories of lighters, n.e.s., other than flints or wicks \\
\hline 89937 & Smoking pipes (including pipe bowls) and cigar or cigarette holders, and parts thereof \\
\hline 89949 & Parts, trimmings and accessories of umbrellas, walking-sticks, seat-sticks, whips, riding-crops and the like \\
\hline 89966 & Artificial parts of the body \\
\hline 89984 & Button moulds and other parts of buttons; button blanks \\
\hline 89986 & Parts of slide fasteners \\
\hline 89996 & Parachutes (including dirigible parachutes) and rotochutes: parts thereof and accessories thereto \\
\hline 89997 & Vacuum flasks and other vacuum vessels, complete with cases; parts thereof (other than glass inners) \\
\hline
\end{tabular}

* Number of five digit products: 225 (SITC 7: 168 and SITC 8: 57) 